\title{
LLNL-TR-570412
}
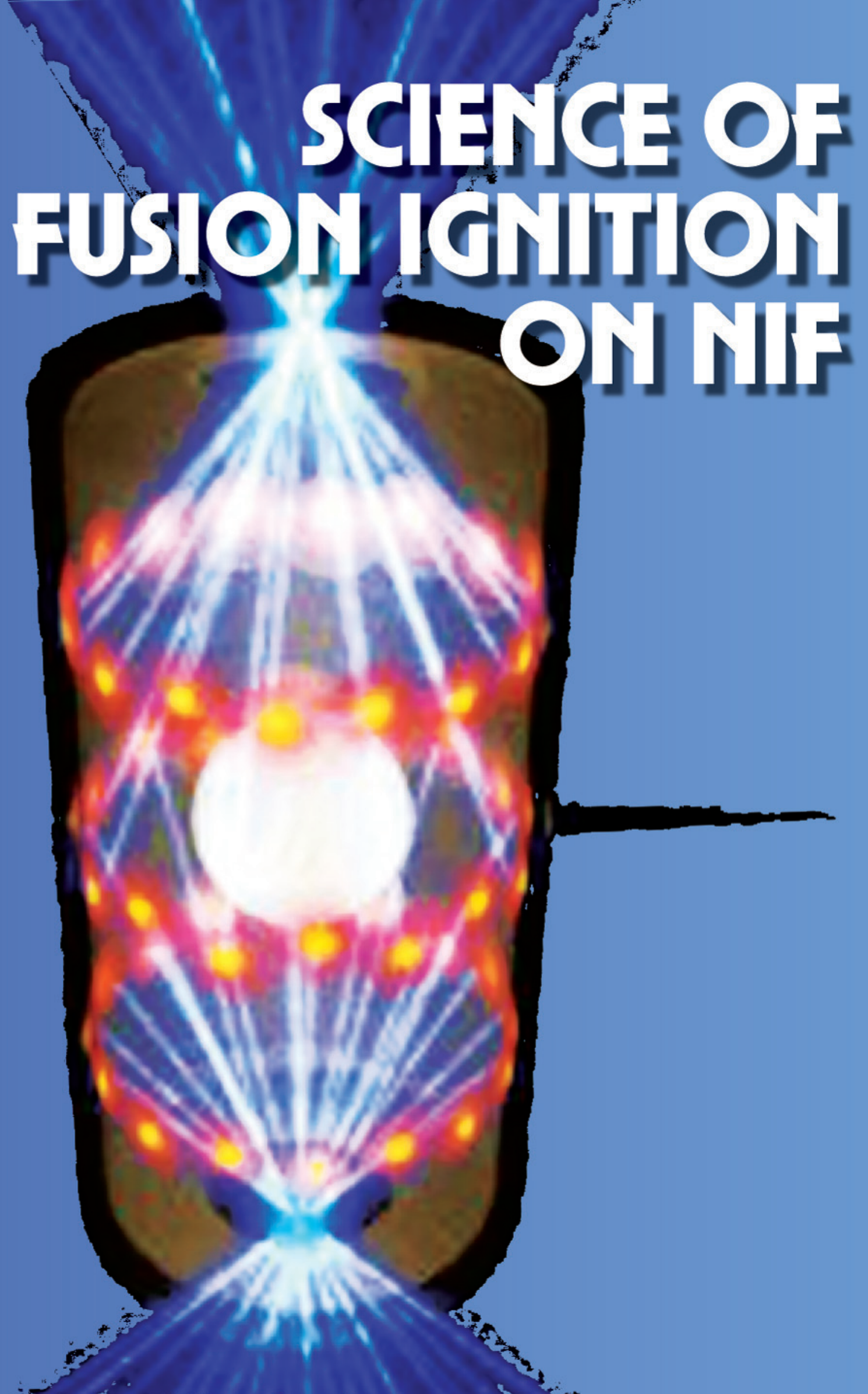

Workshop is Workshop

\section{S $x^{4}$ a} Min 45 


\section{Disclaimer}

This document was prepared as an account of work sponsored by an agency of the United States government. Neither the United States government nor Lawrence Livermore National Security, LLC, nor any of their employees makes any warranty, expressed or implied, or assumes any legal liability or responsibility for the accuracy, completeness, or usefulness of any information, apparatus, produ t, or process disclosed, or represents that its use would not infringe privately owned rights. Reference herein to any specific commercial product, process, or service by trade name, trademark, manufacturer, or otherwise does not necessarily constitute or imply its endorsement, recommendation, or favoring by the United States government or Lawrence Livermore National Security, LLC. The views and opinions of authors expressed herein do not necessarily state or reflect those of the United States government or Lawrence Livermore National Security, LLC, and shall not be used for advertising or product endorsement purposes.

\section{Auspices Statement}

This work performed under the auspices of the U.S. Department of Energy by Lawrence Livermore National Laboratory under Contract DE-AC52-07NA27344.

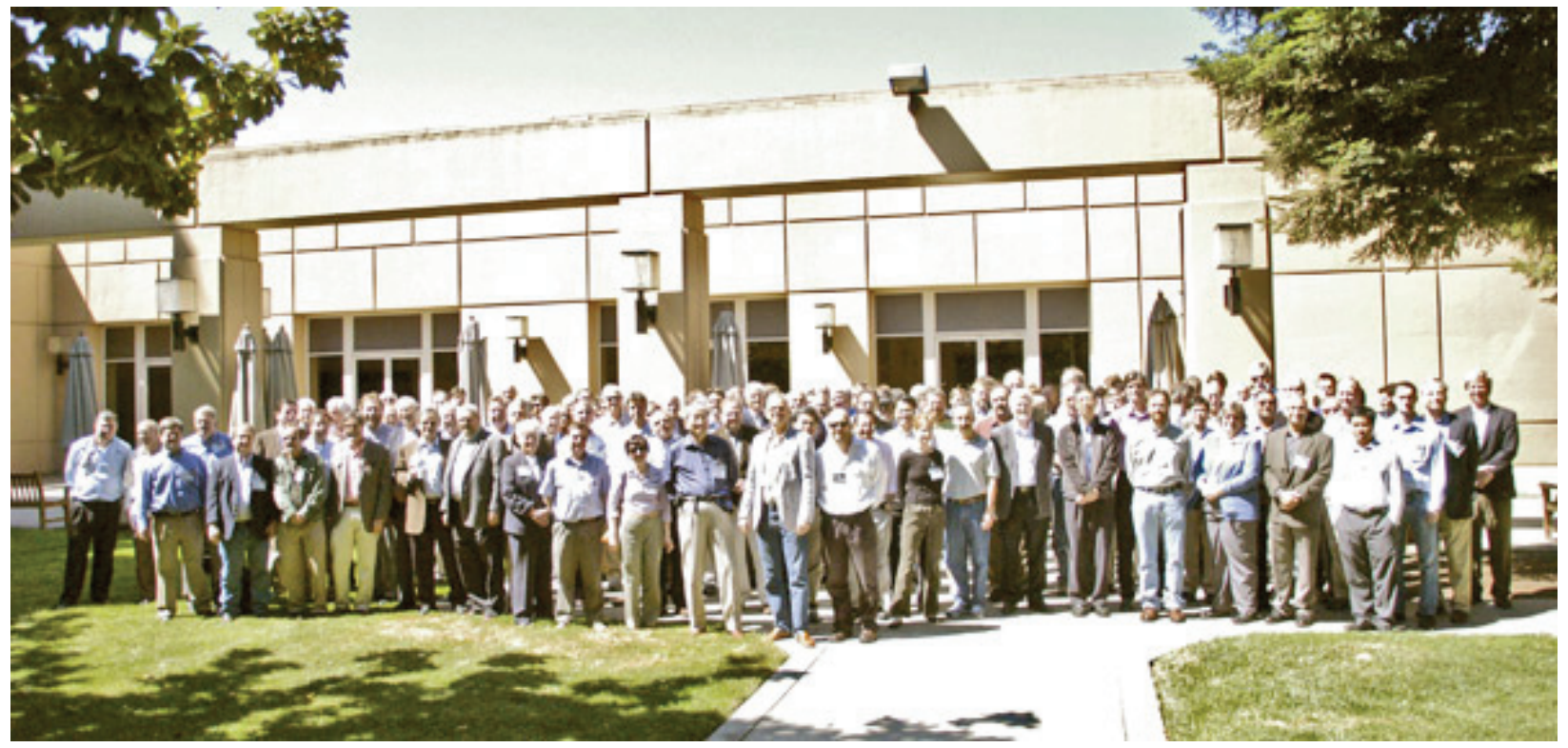

Science of Fusion Ignition on NIF Workshop attendees 


\section{WORKSHOP ON THE SCIENCE OF FUSION IGNITION ON NIF}

Co-Chairs: $\quad$ William Goldstein, Lawrence Livermore National Laboratory Robert Rosner, University of Chicago

Steering group: Cris Barnes, Los Alamos National Laboratory

Riccardo Betti, University of Rochester

Gilbert Collins, Lawrence Livermore National Laboratory

Don Correll, Lawrence Livermore National Laboratory

John Edwards, Lawrence Livermore National Laboratory

Mark Herrmann, Sandia National Laboratories

Craig Sangster, Laboratory for Laser Energetics

Alan Wan, Lawrence Livermore National Laboratory

Alan Wootton, University of Texas

Panel Leads: $\quad$ Laser Propagation and X-ray Generation

Chan Joshi, University of California Los Angeles

Mordecai Rosen, Lawrence Livermore National Laboratory

$X$-ray Transport and Ablation Physics

Jim Hammer, Lawrence Livermore National Laboratory

David Meyerhofer, University of Rochester

Implosion Hydrodynamics

Valeri Goncharov, University of Rochester

Omar Hurricane, Lawrence Livermore National Laboratory

Stagnation Properties and Burn

Riccardo Betti, University of Rochester

Johan Frenje, Massachusetts Institute of Technology

HED Properties and Processes

Gilbert Collins, Lawrence Livermore National Laboratory

Justin Wark, University of Oxford

Integrated Modeling

Don Lamb, University of Chicago

Michael Marinak, Lawrence Livermore National Laboratory 
This page intentionally left blank 


\section{TABLE of CONTENTS}

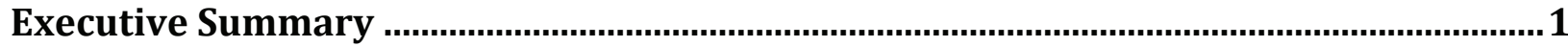

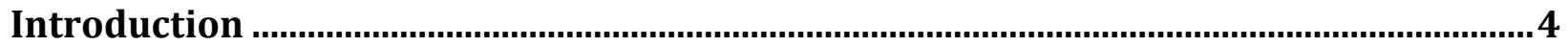

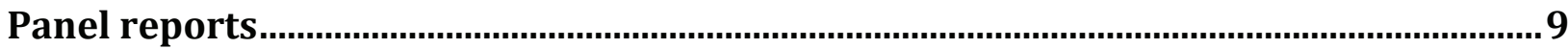

1.0 Panel 1 — Laser Propagation and X-ray Generation.............................................. 10

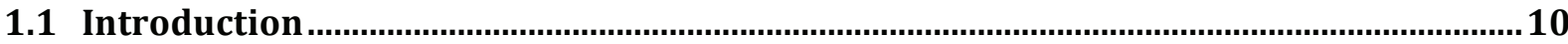

1.2 Status of the Physics .....................................................................................................10

1.2.1 Underlying processes and properties ....................................................................................... 10

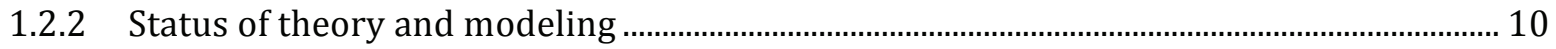

1.2.3 Impact of experimental results...................................................................................................... 11

1.3 Opportunities for Progress ................................................................................................12

1.4 Priority Research Directions......................................................................................13

1.4.1 Hohlraum Plasma Characterization........................................................................................... 13

1.4.2 Electron Plasma Wave Science................................................................................................... 15

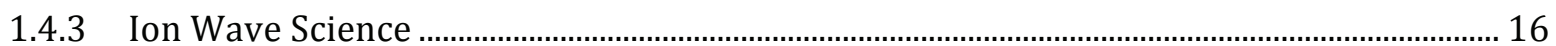

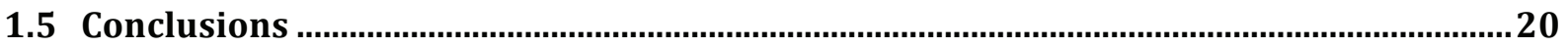

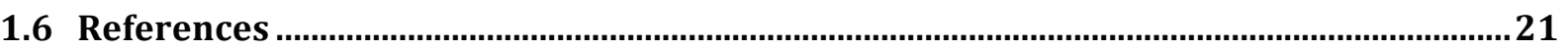

2.0 Panel 2 - X-ray Transport and Ablation Physics ...................................................... 22

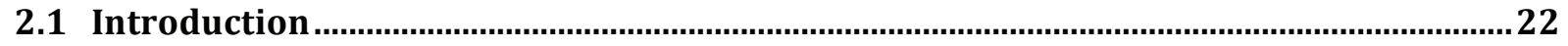

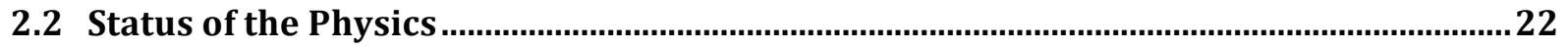

2.3 Opportunities for Progress ............................................................................................23

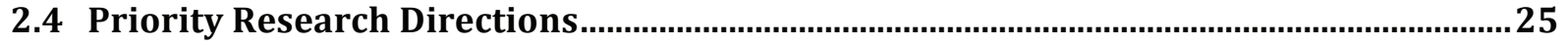

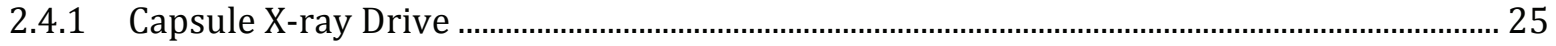

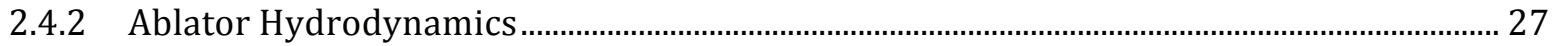

2.4.3 Ablator Radiative Coupling........................................................................................................... 28

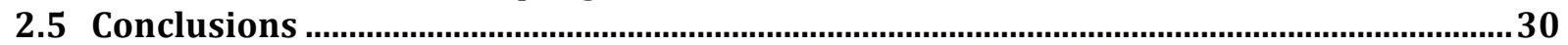

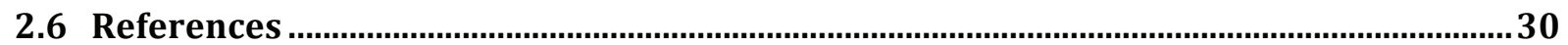

3.0 Panel 3 - Implosion Hydrodynamics....................................................................... 31

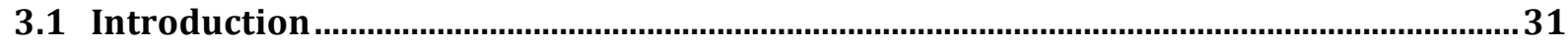

3.2 Status of the Physics ....................................................................................................... 31

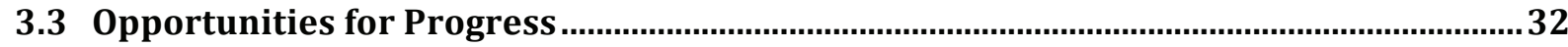

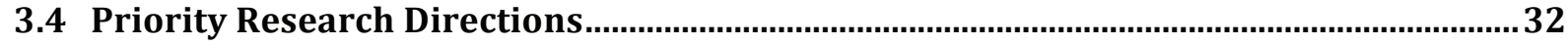

3.4.1 Investigation and Control of Ablation Front Instability .................................................... 32

3.4.2 Mix in Extreme High-Acceleration Implosions Driven by Multiple Strong Shocks........... 34

3.4.3 Hotspot Formation and Fuel-Shape Physics ........................................................................... 35

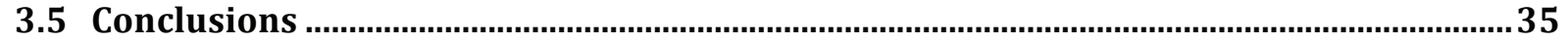

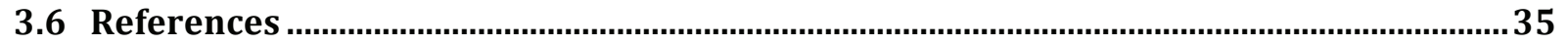

4.0 Panel 4 - Stagnation Properties and Burn ................................................................. 37

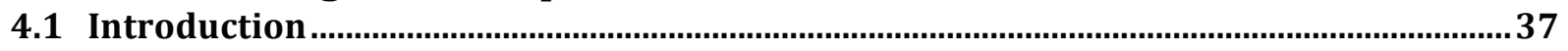

4.2 Status of the Physics .........................................................................................................37

4.2.1 Underlying processes and properties ........................................................................................ 37

4.2.2 Status of theory and modeling …………………………………………………………………….. 37

4.2.3 Impact of experimental results................................................................................................ 38

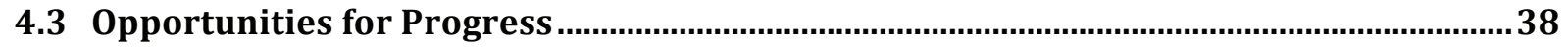

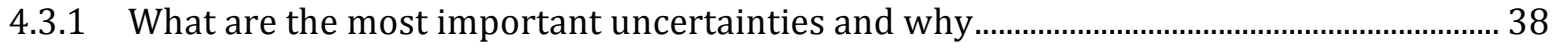

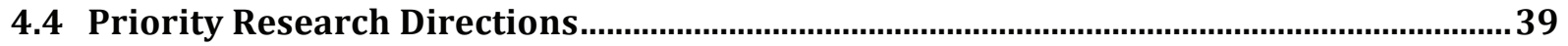




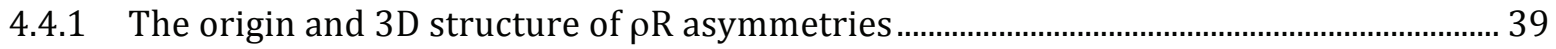

4.4.2 In-flight characteristics of the DT fuel ……......................................................................... 43

4.4.3 Probing energy balance at stagnation ...................................................................................... 45

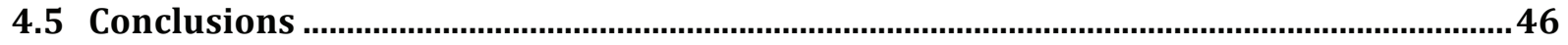

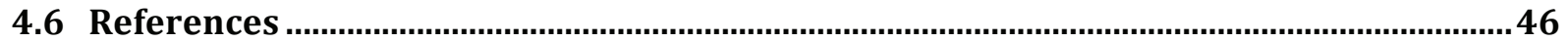

5.0 Panel 5 - HED Properties and Processes.............................................................. 47

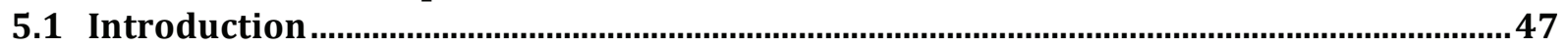

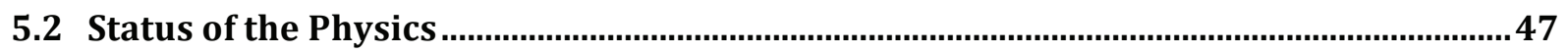

5.3 Priority Research Directions..............................................................................................55

5.3.1 Equations of state for ultra-dense matter and conduction dominated "gas

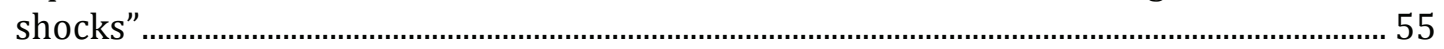

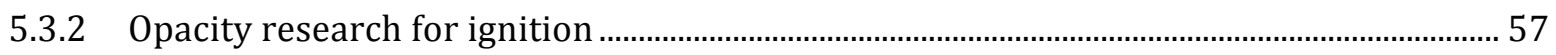

5.3.3 Develop an understanding of transport and kinetic phenomena in ignition..................... 60

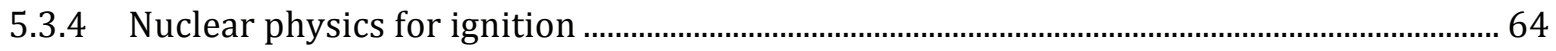

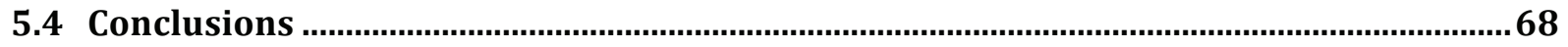

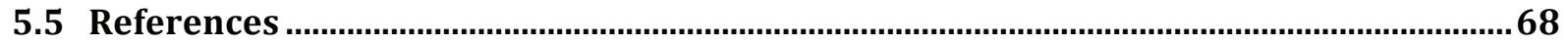

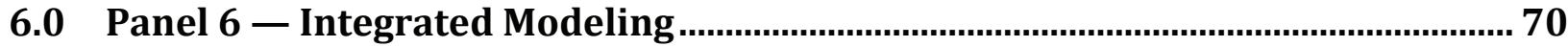

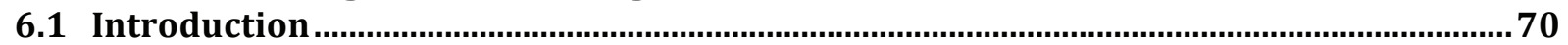

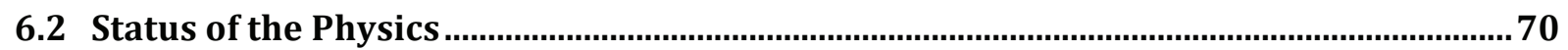

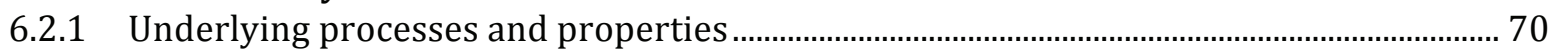

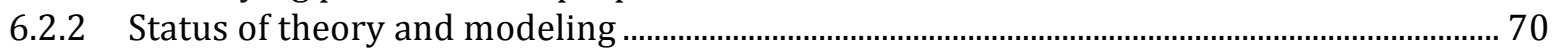

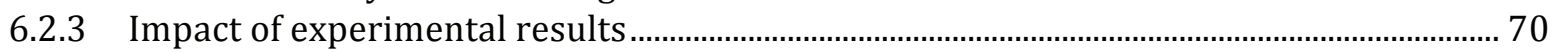

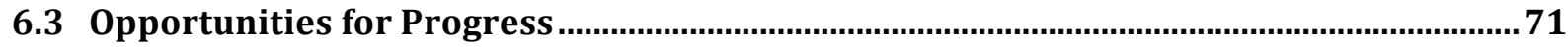

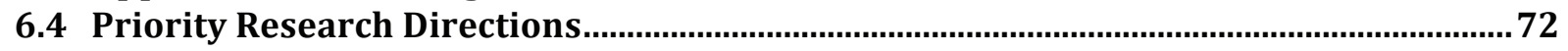

6.4.1 A Science-Based Validation Campaign........................................................................................ 72

6.4.2 Improved Modeling of Hohlraum Energetics ………................................................................ 76

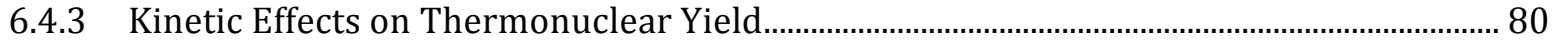

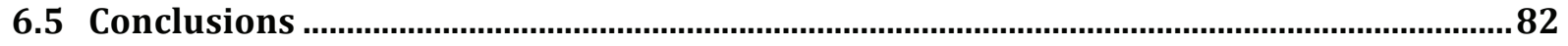

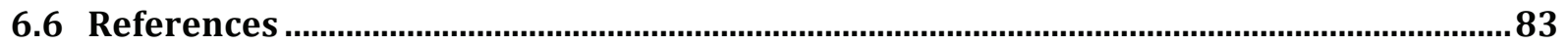

Appendix A: Agenda ............................................................................................................. 85

Appendix B: Workshop Participants................................................................................ 87

Appendix C: Panel Scope Descriptions ….................................................................... 91

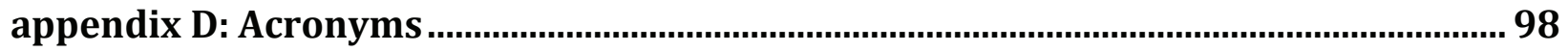

Appendix E: Bibliography .................................................................................................. 100 


\section{Sidebars}

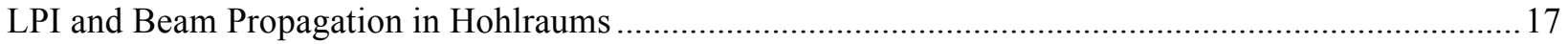

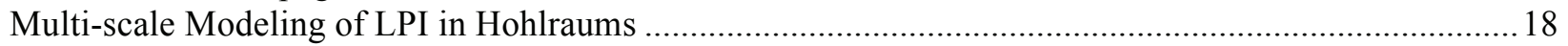

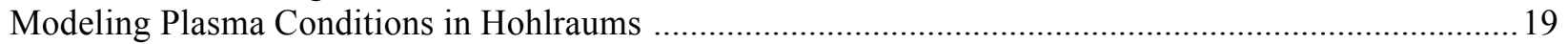

Continuous Improvements to Modeling Plasma Conditions in Hohlraums ............................................20

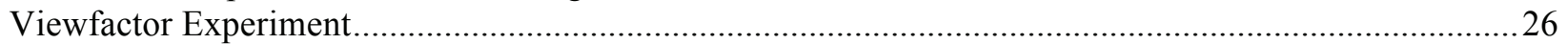

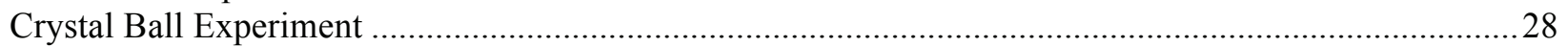

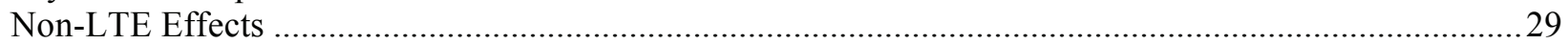

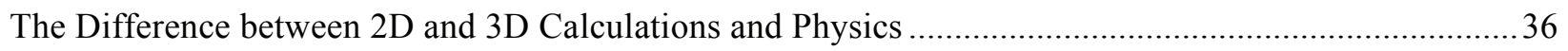

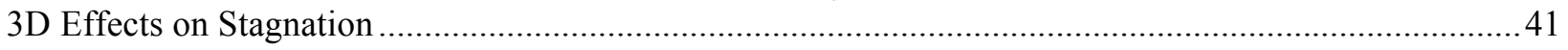

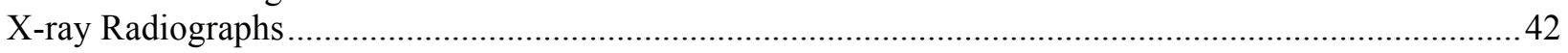

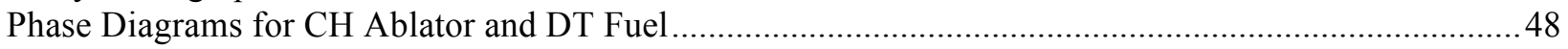

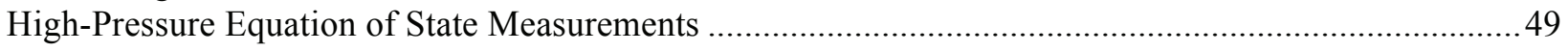

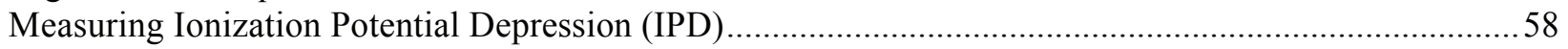

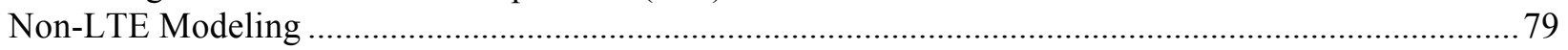

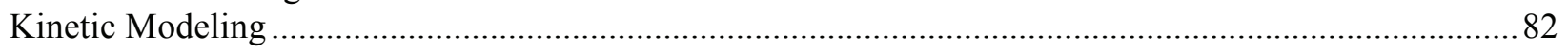

\section{Figures}

Figure 3.1: Altering the first picket in the drive (upper left) potentially shifts the extrema of the ablation from instability growth (upper right) without much impact on fuel adiabat. Increasing and shorting the foot level of the "trough" of the drive pulse (lower left) potentially reduces the instability growth for many mode numbers (lower right). Figures courtesy of the NIC target design team.

Figure 3.2: A 2D mode 100 ARES simulation of an ICF implosion with realistic surface roughness shows the expected late-time implosion morphology and hotspot. Prior to self-heating from alpha-particle deposition, mechanical pdV work produces heating that spreads radially outward into the DT fuel via electron conduction (assuming conduction and radiation losses don't dominate). This ablated fuel forms the hot spot and is many times the mass of the DT gas that occupies the volume at the center

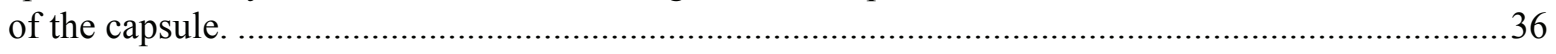

Figure 6.1: Schematic of the geometry for experiments with face-on radiography .................................75 Figure 6.2: Power is transferred from laser beam 0 to beam 1 when the beat wave's phase velocity $\mathrm{v}_{\phi}=\Delta \omega / \Delta \mathrm{k}$ is near the plasma acoustic speed $\mathrm{C}_{\mathrm{s}}$. The process is tunable via $\Delta \omega$, the frequency difference between the two laser beams. 
This page intentionally left blank 


\section{EXECUTIVE SUMMARY}

This report summarizes the discussions and findings of a workshop held in May 2012, to discuss science that has been learned in the National Ignition Campaign, identify new science questions that have arisen, and begin to lay the lines of experimental and theoretical inquiry that could address these over a multi-year time frame.

Scientists meeting over two days in San Ramon, CA, worked in six panels. Four panels covered the stages of an ignition experiment, a fifth looked at cross-cutting high-energy density materials issues, and a sixth considered the integrated, multi-physics modeling codes. Each panel identified priorities for future research that will enhance our understanding of ignition and the unique conditions upon which it depends.

Panel 1 considered the physics of laser propagation in the ignition hohlraum and the generation of the $\mathrm{x}$-ray flux that drives the implosion of the fuel capsule. This panel focused on laser plasma interactions, including collisional absorption, backscatter and cross-beam energy transfer. The latter effect - though incompletely understood - is relied on to achieve a symmetric hotspot. The panel noted that recent experiments have indicated that the hohlraum plasma is cooler than originally predicted, that ad hoc multipliers applied to the laser drive to bring simulations into agreement with measured implosion velocities are not consistent with the measured $x$-ray flux exiting the laser entrance holes, and that laserplasma interaction (LPI) effects not included in current simulations may be contributing to low neutron yields and other unexpected phenomena. The observation of lower hohlraum temperatures led to the implementation of the "so-called" high flux model (HFM), which replaced standard treatments of the plasma atomic kinetics and nonlocal heat transport with new models presumed to be more accurate. The panel also noted, though, that the HFM has not yet been experimentally validated and called for an effort to directly measure plasma conditions in the hohlraum. In addition to helping validate the HFM, this proposal, identified by the panel as its first priority research direction (PRD), would provide a firm basis to improve our understanding of LPI and help to elucidate the origin of the laser power multipliers needed to match implosion dynamics. The panel's second and third PRDs make the case for improving our understanding of simulated Raman and Brillouin scattering (SRS and SBS) that limit the conversion of laser energy to $\mathrm{x}$-rays and mediate the transfer of energy between beams. Among the panel's specific recommendations were a call for higher resolution simulations of hohlraum dynamics, the implementation of better models of non-local thermodynamic equilibrium (non-LTE) atomic physics and LPI, and the exploration of point designs with higher hohlraum electron temperatures to minimize stimulated Raman backscattering.

Panel 2 took up the subject of $x$-ray propagation in the hohlraum and the physics of ablation that drives the implosion of the fuel capsule. The directions identified by this panel were driven primarily by the discrepancy between the measured implosion velocities and simulations based on the X-ray drive measured by the Dante diagnostic. Two hypotheses are suggested for this discrepancy: either the measured drive is not that "seen" by the capsule, or the ablation process is not being accurately modeled. Of course, both effects may be relevant, and the PRDs identified by the panel address both the drive and the ablation process, as well as ablation effects such as instabilities, mix, non-radial flow, and additional shocks, which can impact yield. To resolve these possibilities, the panel recommended as its highest priority a measurement of the x-ray flux at the ablator. Subsequent priorities depend on whether this flux is consistent with the velocity or not. If it is - that is, if the x-ray drive is weaker than predicted - the next steps would be to understand why, through detailed study of hohlraum energetics and x-ray transport. If not, the priority would be to develop a predictive understanding of the coupling of radiation to ablator materials, based on detailed investigations 
of opacity, non-LTE effects, equation of state (EOS), thermal transport and pre-heat.

Panel 3 looked at the hydrodynamic processes taking place during the implosion. The challenge of obtaining high convergence while avoiding instability growth and mix frame the research directions identified by this group. Noting that the pressure drive, the onset of mix, and the stagnation pressure are currently not adequately modeled, the scientific goal of improving the predictive power of simulations is highlighted. Fundamental physical processes that can impact the accuracy of simulations include the growth rate of ablation front instabilities, thermal conductivity, and parameterization of mix and its dependence on initial conditions. The first PRD lays out a series of experiments designed to "take apart" the physics of the ablation front instability by taking advantage of $1 \mathrm{D}$ geometries, by varying materials and by scaling in energy. Systematically comparing these to simulations would help identify gaps in models. The second PRD addresses hotspot mix both through experimental studies of sensitivity and scaling and through the development of an improved sub-grid model. The deceleration phase of the implosion is the focus of the third PRD, which describes potential steps to improve the data on the state of the cold fuel, higher accuracy simulations, and improvements in relevant physics processes such as conductivity.

Panel 4 examined the implosion physics that controls temperature, density, and $\rho \mathrm{R}$ in the hotspot at stagnation and determines if they are sufficient for alpha heating and burn. The PRDs identified by the panel encompass the in-flight conditions of the cold fuel and central hot gas, the hydrodynamic response to capsule and hohlraum 3D asymmetries, and the energy partitioning and transport within and between the hotspot and cold fuel, factors that ultimately combine to determine the assembled hotspot and fuel conditions at stagnation. Priorities were set based on analysis of cryogenically layered capsule implosions that indicate, first and foremost, that, even when implosion modeling is tuned to reproduce shock timing and implosion velocity measurements, the observed stagnation pressure of the hotspot and cold fuel are significantly lower than predicted, resulting in low yields. This pressure deficit, given the experimental evidence, is most likely $3 \mathrm{D}$ in nature and associated with an incomplete conversion of the kinetic energy of fuel shell into compression of the hotspot. Low-mode $\rho \mathrm{R}$ asymmetries, which are generally observed in cryogenic layered implosions, can reduce the pressure at stagnation and also increase the onset of hotspot mix. The first PRD is to understand the origin and $3 \mathrm{D}$ structure of the $\rho \mathrm{R}$ asymmetries, with an emphasis on direct measurements of the development and growth of these features in time, through the use of radiography both in-flight and at stagnation. The second PRD is aimed at a better understanding of the in-flight characteristics of the DT fuel, and in particular, those properties that determine the density and energy in the hotspot as it is formed, which, along with the velocity and cold fuel adiabat, determine the stagnation pressure in 1D. Experiments and modeling are outlined to better understand the central gas density, the DT EOS and release states, the sources of preheat, and the sources and effects of "coasting," the tendency of the capsule to decouple from the radiation before stagnation. These effects could reduce the stagnation pressure, but they also result in lower convergence and lower $\rho \mathrm{R}$ than is observed experimentally. Finally, the third PRD is to develop methods such as radiography and spectroscopy that can directly probe the energy balance and density distribution in the hotspot and fuel at stagnation, with an emphasis on examining the possibility of anomalous radiative and conductive heat transfer between hotspot and cold fuel. This depends on the conditions of the cold fuel and could affect mass ablation into the hotspot, if conditions inhibit the absorption of hotspot radiation.

Panel 5 considered underlying high energy density properties and processes, including EOS, opacity, transport, kinetics, and nuclear interactions. These phenomena control all aspects of an igniting target: laser transport, $\mathrm{x}$-ray generation, ablation, shock timing, instability growth, hotspot formation, and burn. While many processes are represented in the simulations, they have generally not been experimentally tested over the range of conditions attained in ignition experiments. In addition, a number of properties and processes are treated at gross levels of approximation that might obscure their impact. 
The panel used the National Ignition Campaign (NIC) results to guide and help prioritize a set of research directions aimed at improving key physical models used for inertial confinement fusion (ICF) calculations, including EOS, LTE and non-LTE opacity, nuclear reactions, kinetics, electric and magnetic field generation, and several transport quantities such as thermal conduction, electron-ion equilibration, ion stopping power, and viscosity. The unique, heretofore unexplored extremes encountered in the ignition regime lead to very stressing tests of the assumptions that have informed current modeling, for example, the Thomas-Fermi limit for EOS, the Stewart-Pyatt model for the effects of ionization potential depression on opacity, strong shock effects on release states, equilibration, and species separation, which may effect energy balance and transport and plasma effects on nuclear processes. This part of the report independently identifies and collects many of the observations made by the panels that looked at the evolution of the target and makes it clear that there are many opportunities to improve and better test the physics models that underlie ICF.

Panel 6 took a cross-cutting look at integrated modeling and the multi-physics codes that guide ignition target design and help with the interpretation of experimental results. The current simulation capability has been extensively tested against experiments at Shiva, Nova and OMEGA, but recent experiments have clearly called into question the ability of the codes to predict important observables, including yield under the conditions attained in National Ignition Facility (NIF) ignition experiments. Examples - most of which were noted by multiple panels - include the radiation drive in the hohlraum, the need for drive multipliers to match shock timing and implosion velocity measurements, the pressure and density of the hotspot, and, of course, the neutron yields, which are up to ten times lower than predicted. By considering these discrepancies and applying a broad perspective on the codes, the panel identified priorities at three levels of integration: first, treating validation of the codes systematically; second, focusing on getting the integrated modeling of hohlraum energetics correct; and third, including kinetic and field effects on yield. In its first research direction, the panel describes an extensive, systematic sciencebased campaign to validate the models and simulations, including code-to-code comparisons, a hierarchical strategy of experimental validation, and a robust study of sensitivity, including the application of specific uncertainty quantification techniques. In its discussion of the modeling of hohlraum energetics, this panel report touches on and summarizes the issues raised by Panels 1 and 2 , while describing a focused campaign of experiment and code-development. Specific opportunities to improve the simulations include the EOS and opacity models, non-LTE effects, non-local electron transport, laser-entrance hole (LEH) closure, and improved accounting for energy loss owing to SRS and SBS. With respect to the hotspot, the panel emphasizes in a third PRD the use of the best available models to assess sensitivity to and impact of a variety of effects, including barodiffusion, non-local transport, and non-Maxwellian distributions.

Beyond meeting its goal of laying out new lines of research, the workshop was successful in educating and engaging an expanded community of scientists in the compelling science of fusion ignition. Over 150 participants from 6 countries and 40 laboratories and universities vigorously discussed the world's first ignition experiments, challenging results and debating implications. Among the clear conclusions of the meeting was a broad desire to continue the colloquy initiated in San Ramon. 


\section{INTRODUCTION}

The National Ignition Facility (NIF), built by the National Nuclear Security Administration (NNSA) and commissioned in 2009, is the world's most energetic laser. Recently, the NIF successfully delivered $1.9 \mathrm{MJ}$ of ultraviolet light in a 500-TW pulse to an inertial confinement fusion (ICF) target, marking a critical milestone in capability for the NNSA's Stockpile Stewardship Program (SSP). The advent of the NIF allows NNSA scientists to recreate conditions and study processes that occur in nuclear explosions. For the broader science community, NIF presents opportunities to explore what happens at the center of stars, giant planets, and supernovae.

A principle near-term goal of the NIF is to demonstrate break-even fusion burn in an indirectdrive ICF target configuration. The program of ignition experiments at the NIF is the National Ignition Campaign (NIC). Achieving "ignition" will provide NNSA the ability to study in the laboratory phenomena associated with fusion boost, a grand challenge in weapons science. Ignition will also be a key milestone in the development of inertial fusion energy. Demonstrating ignition is a scientific grand challenge that requires creating extreme states of matter under precise, highly controlled conditions. While many of the stages of ignition have been studied in the lead-up to the NIC, using smaller facilities such as Nova and OMEGA, the conditions achieved by ignition experiments at NIF are well beyond anything previously explored. As in any experiment that explores frontiers in physics, surprises are to be expected.

Steady progress has been made towards attaining the conditions required for ignition. At the same time, a wealth of exquisite data has been collected at conditions never before attained in the laboratory, including laser-plasma interactions at unique power densities and scale lengths, terapascal shock propagation, petapascal pressures, and 10,000-fold compressions. New phenomena have been observed, and surprises have arisen in comparisons between experimental results and theory, as represented by the multiphysics simulations used to model experiments.
An international workshop was held in May 2012, to discuss the science that has been learned in the NIC, identify scientific questions that have arisen, and begin to lay the lines of experimental and theoretical inquiry that would build on and address these over a multi-year time frame. Specifically, the goals of the workshop were to:

- Engage and expand the community of scientists interested in exploring science of ignition on NIF

- Form the basis for future efforts to explore underlying physics needed to understand ignition designs for a range of applications

- Identify paths leading to improved integrated design capabilities

- Maximize the utility of NIC results for broader ICF/inertial fusion energy (IFE) community

Over 150 scientists met in San Ramon, CA, over two days, working in six panels. Four panels covered the stages of an ignition experiment, a fifth looked at cross-cutting high-energy density materials issues, and a sixth considered the integrated, multi-physics modeling codes. The panels were asked to:

- Identify the key physics that underlie indirectdrive inertial fusion ignition

- Review and summarize our understanding of this key physics, including new insights and questions raised by recent experimental results

- Propose research directions that would address continuing gaps in understanding key physics

- Assess the likely impact of each of these modeling or experimental areas in furthering progress in understanding ignition science

This report summarizes the discussions and findings of the workshop. Its six main sections represent the response of each panel to the workshop charge. Each panel was able to identify priorities for future research that will enhance our understanding of ignition and the unique conditions upon which it depends. These "Priority Research Directions" are listed in Table 1. A summary of the scope and findings of each panel is given below. 
Table 1. Summary of the priority research directions.

\begin{tabular}{|c|c|}
\hline Panel & Priority Research Directions \\
\hline \multirow{3}{*}{$\begin{array}{l}\text { 1. Laser } \\
\text { propagation } \\
\text { and X-ray } \\
\text { production }\end{array}$} & Hohlraum plasma characterization \\
\hline & Electron plasma wave science \\
\hline & Ion wave science \\
\hline \multirow{3}{*}{$\begin{array}{l}\text { 2. X-ray } \\
\text { transport and } \\
\text { ablation } \\
\text { physics }\end{array}$} & Capsule x-ray drive \\
\hline & Ablator hydrodynamics \\
\hline & Ablator radiative coupling \\
\hline \multirow{3}{*}{$\begin{array}{l}\text { 3. Implosion } \\
\text { hydro- } \\
\text { dynamics }\end{array}$} & Investigation and control of ablator front instability \\
\hline & Mix in extreme high-acceleration implosions driven by multiple strong shocks \\
\hline & Hotspot formation and fuel shape physics \\
\hline \multirow{3}{*}{$\begin{array}{l}\text { 4. Stagnation } \\
\text { and burn }\end{array}$} & The origin and $3 \mathrm{D}$ structure of $\rho \mathrm{R}$ asymmetries \\
\hline & In-flight characteristics of the DT fuel \\
\hline & Probing energy balance at stagnation \\
\hline \multirow{4}{*}{$\begin{array}{l}\text { 5. HED } \\
\text { Properties } \\
\text { and } \\
\text { Processes }\end{array}$} & Equations of state for ultra-dense matter and conduction dominated "gas shocks" \\
\hline & LTE and non-LTE opacity research for ignition \\
\hline & Develop an understanding of transport and kinetic phenomena in ignition \\
\hline & Nuclear science for ignition \\
\hline \multirow{3}{*}{$\begin{array}{l}\text { 6. Integrated } \\
\text { modeling }\end{array}$} & A science-based validation campaign \\
\hline & Improved modeling of hohlraum energetics \\
\hline & Kinetic effects on thermonuclear yield \\
\hline
\end{tabular}

\section{Panel 1: Laser propagation and $x$-ray production}

Laser propagation and $x$-ray production refers to the physics of the laser beams entering the hohlraum and interacting with the hohlraum walls and the subsequent $x$-ray production. The "outer" NIF laser beams enter the hohlraum, propagate towards, and primarily heat the $\mathrm{Au}$ wall. They are collisionally absorbed in subcritical gold plasma, and electron thermal conduction then heats denser gold, which is the primary source of $\mathrm{x}$-ray production. The $\mathrm{x}$-rays are mostly sub-keV ("capsule drive") photons, plus "M-band" (2-4 keV) photons that have capsule preheat implications. The "inner" beams, on their way to the Au wall near the hohlraum midplane, traverse a longer plasma path, much of which consists of both low-Z "hohlraum fill gas" as well as rarefied, heated, capsule ablator material.
Many fundamental physical processes are involved. Beam overlap in the plasma results in time-dependent transfer of power from outer to inner beams, which is being used to control the capsule implosion symmetry. Accurately calculating this crossbeam transfer requires knowledge of the plasma conditions (temperature $\mathrm{T}$, density $\mathrm{n}$, velocity field $\mathrm{v}$ ) near the laser entrance hole (LEH) and a theory of the saturation mechanisms for the transfer. As the beams propagate, they are collisionally absorbed. Accurately calculating this absorption again requires knowledge of the plasma $\mathrm{T}, \mathrm{n}$, and charge state Z. Non-local thermodynamic equilibrium (Non-LTE) radiation processes, non-local electron conduction, and non-Maxwellian particle distributions all affect these quantities. The mixing of the high-Z Au blow-off plasma from the wall with the low-Z fill gas plasma can also 
affect these quantities. The LEH itself is dynamically closing and is difficult to model properly, and magnetic fields are likely to form within its sharp gradients. This is important because the LEH affects laser beam propagation inward, and the outward x-rays from which the $\mathrm{x}$ ray drive is deduced.

Laser-plasma instabilities (LPI) occur, and stimulated Brillouin scattering (SBS, laser light scattering from ion waves) and stimulated Raman scattering (SRS, laser light scattering from electron waves) are observed. The SRS electron plasma wave can produce hot electrons that can either directly or indirectly preheat the capsule. Accurately calculating these LPI processes has been a multi-decade and multi-scale enterprise, requiring at least a detailed knowledge of both local plasma and local laser light parameters.

This Panel emphasizes that improved knowledge of local plasma parameters, using diagnostics techniques already proven elsewhere, is within reach. The objective is a fundamental understanding of the basic plasma and beamplasma processes involved. This fundamental understanding, in conjunction with changing (improving) the plasma conditions, should allow mitigation of SRS and SBS and control of cross beam energy transfer.

\section{Panel 2: X-ray transport and ablation physics}

$\mathrm{X}$-ray transport and ablation physics refers to the physics of the processes that drive the "1D" or spherically symmetric component of the hydrodynamics of the implosions. This is the $\mathrm{x}-$ ray transport within an indirect-drive hohlraum and the subsequent ablation of the capsule. Of particular interest are the coupling efficiency to, and the drive pressure history of, the capsule.

The NIC capsule implosion experiments currently show significant differences between the predictions of models employing the nominal $\mathrm{x}$ ray drive and the observed hydrodynamic properties such as velocity history, bang time and shell thickness. Inferred stagnation pressures are lower than predicted, suggesting that extra entropy is injected into the capsule hotspot that in turn inhibits compression, possibly a result of the hydrodynamics. These differences affect every aspect of the implosion-symmetry, stability, and mix. Understanding the underlying physics and developing predictive models will allow the design of more optimal implosions. Research directions explore possible explanations for these discrepancies. The $\mathrm{x}$-ray drive seen by the capsule might be different than what is measured in the hohlraum; or the response of the ablator might be incorrectly predicted in simulations.

Concerning the $\mathrm{x}$-ray drive and an apparent missing energy component, recent data suggest that models may over-predict LEH closure, so the actual radiation temperatures through the LEH and on the capsule are lower than simulated. Concerning the ablator material properties, which play a crucial role in capsule performance, some are based on theoretical models and need experimental validation in the relevant regimes. Different EOS and opacity models as well as potential non-LTE properties of the polymer ablator remain of active interest, with consequences for energy balance and implosion time.

The highest priority research in this area is to understand the experimental relationship between the $\mathrm{x}$-ray drive and the implosion velocity and the consistency with expectations (Table 1, research direction 2.1). The outcome determines the subsequent relative importance of other priority research directions; is it the drive-production physics or is it the ablator physics that is most important?

\section{Panel 3: Implosion hydrodynamics}

Implosion hydrodynamics refers to the physics specifically associated with the capsule implosion on the NIF, driven by a four-step laser intensity waveform (4 "pickets"). Current relevant experimental observations include: an ablation pressure history that is inconsistent with predictions, a hotspot pressure (at stagnation) and neutron yield lower than predicted, mix (between fuel and ablator material) occurring at lower implosion velocities than predicted, and asymmetries in the cold fuel. To match the shock timing data with direct numerical simulations requires using time-dependent reduction factors on the laser energy. Thus, the drive pressure history is uncertain as a function of solid angle and time, as is any preheat of the capsule. The physics opportunities related to understanding 
these uncertainties in the implosion initial boundary conditions is covered in the first two panels. However, even when a best effort is made to account for the 'initial condition' uncertainties, the experimental stagnation pressure and neutron yield are not accurately predicted. This suggests that interesting physics is at play within the implosion process itself.

The boundary conditions for the implosion hydrodynamics can, in addition to affecting the apparent implosion efficiency, affect the fuel adiabat and the mix (of ablator material with fuel). A non-optimal x-ray drive can produce a "fifth shock" and also result in shell decompression. A lower-than-predicted neutron yield and stagnation pressure can result from an increase in the volume of the vapor region due to multi-dimensional effects (low-mode hotspot distortion and mix). Finally, current calculations of the hotspot formation do not include the effects of magnetic fields on electron heat conduction, the primary process behind formation of the hotspot.

Thus, the physics specifically related to implosion hydrodynamics considered in panel 3 is the physics of instability, mix, and hotspot formation by ablation of the DT ice inner surface. Research opportunities exist in all three areas, and it is important to understand the role of threedimensional effects. One experimental approach suggested is to vary target and $\mathrm{x}$-ray drive parameters to elucidate the role of different fundamental physics properties.

\section{Panel 4: Stagnation and burn}

Stagnation and burn refers to the essential conditions for a successful fuel assembly and, thus, the achievement of hotspot ignition and energy gain. A consideration in this section is an assessment of the models used to infer properties at stagnation, in particular, a two-radial-zone, otherwise $0 \mathrm{D}$, interpretive model that uses all available data for input to predict final parameters. There is evidence for mixing of ablator material with the fuel in the hotspot for cold fuel asymmetries and for unexpectedly low energy transport between hot and cold dense regions. Fundamental research in the areas of 3D perturbations and their consequences and energy transport at high densities is needed to explain the observations.
Basic considerations show that one explanation for a low stagnation pressure is a high DT gas density present at stagnation, resulting from an increased mass being compressed. The stagnation pressure is also affected by shock timing (itself dependent on material properties and perhaps kinetic effects), by any additional shock, and by perturbations to sphericity of either a low or high mode number. All these are covered by the priority research directions, which include suggestions for additional diagnostics of the stagnated plasma conditions.

\section{Panel 5: High energy density properties and processes}

High energy density properties and processes refers to the materials data needed to simulate and interpret ignition implosion experimental data. Considerations include evolving a next-generation of benchmarked physics models expressly focused on the interpretation of experiments and optimization of future fusion designs. Physics areas include EOS, opacity (LTE and non-LTE), nuclear cross sections, kinetics, electric and magnetic field generation, and several transport quantities such as thermal conduction, electron-ion equilibration, ion stopping power, and viscosity. Most models for underlying physics used in ICF designs remain untested over the full range of extreme conditions accessed by the ICF implosion. Priority research in four areas is identified: EOS, LTE and nonLTE opacity, transport and kinetics, and nuclear science.

\section{Panel 6: Integrated modeling}

Integrated modeling refers to the simulation codes currently used to design and analyze ignition experiments. Physical processes modeled in the codes include radiation transport, electron and ion thermal transport, thermonuclear burn and transport of burn products. These codes include models for transport of laser light and the various processes that affect it. They also include magnetohydrodynamics and the effects of magnetic fields on transport processes. Ingredients in the integrated models include atomic physics, in particular, models for opacities and equations of state, both LTE and non-LTE. Approaches that include kinetic and non-fluid effects are being investigated. 
Considerations include developments that would have the greatest impact on modeling ignition experiments, the exploitation of future computational platforms, the approximations made or processes not treated significantly that affect the accuracy, new approaches for modeling physical processes that would substantially benefit the simulation codes, and improved verification and validation. The priority research directions are: instigate a science-based code validation campaign, improve the modeling of hohlraum energetics, and investigate possible kinetic effects on thermonuclear yield.

In presenting the conclusions of these six panels, this report is designed to provide actionable input to the development of a multiyear research program on the physics of indirectdrive fusion ignition. While there are obvious overlaps in the issues addressed and research directions identified by each panel, together they capture the status of ignition science at NIF and point a clear path forward. 


\section{PANEL REPORTS}




\subsection{PANEL 1 - LASER PROPAGATION AND X-RAY GENERATION}

\subsection{Introduction}

In experiments designed for the National Ignition Campaign (NIC), laser beams propagate through hohlraum target laser entrance holes (LEHs) and proceed toward the hohlraums' interior gold walls that absorb the light and convert thermal energy to x-rays used to drive the capsule. This panel deals with the issues that initiate the sequence of events that eventually lead to capsule implosion and ignition. Understanding the partitioning of the laser energy into absorption, scattering and hot electrons is critical not only for the overall energetics, but also for predicting the x-ray drive that ultimately determines the implosion symmetry, velocity and possible preheat of the ablator and core. The purpose of this panel is to identify key science issues and needs, that when properly addressed, could help us propose strategies to minimize laser-plasma instabilities, make cross beam energy transfer an optional rather than a necessary tool, and have a more predictive handle on symmetry of x-ray illumination.

\subsection{Status of the Physics}

\subsubsection{Underlying processes and properties}

There are many issues to consider in properly modeling the large number of physical processes involved here. Due to beam overlap in the plasma, there is usually transfer of power from outer to inner beams, called "cross beam energy transfer" (CBET). This time dependent energy transfer is being used for capsule implosion symmetry control. Accurately calculating CBET requires knowledge of the laser and plasma conditions ( $\mathrm{T}, \mathrm{n}$, velocity field) near the LEHs, as well as a good theory for the saturation mechanisms of the transfer.

As the beams propagate, they are collisionally absorbed. Accurately calculating the absorption also requires knowledge of the $\mathrm{T}, \mathrm{n}$, and $\mathrm{Z}$ of the plasma. The non-local thermodynamic equilibrium (non-LTE) radiation processes, the non-local electron conduction, and the non-Maxwellian particle distributions all affect these quantities. Mixing of the high-Z Au blowoff plasma from the wall with the low-Z fill gas plasma can also be a factor in determining these quantities. The LEH itself is dynamically closing and difficult to model properly, and magnetic fields are likely to form within its sharp gradients. The LEH affects laser beam propagation inward, as well as "Dante", the x-ray drive diagnostic instrument that gathers X-rays emitted through that LEH and is used as an indicator (via comparisons with rad hydro codes) of what the capsule drive may be.

Laser-plasma instabilities (LPI) also occur [1]. Stimulated Brillouin scattering (SBS) and stimulated Raman scattering (SRS) instabilities scatter the light off of self-generated ion acoustic and electron plasma waves, respectively. The SRS electron plasma wave can produce hot electrons that can preheat the capsule directly, or possibly indirectly through inducing highenergy photons from the Au wall. Accurately calculating these LPI processes has been a multidecade and multi-scale enterprise. Accurately knowing the plasma conditions and the local laser intensity are necessary (though possibly insufficient) inputs to understanding what LPI to expect.

As one example of the non-linear cross talk amongst all of the above, consider this: crossbeam transfer can intensify just a part of an inner beam. The resulting SRS from that intense part of the inner beam can send light back from the interior of the hohlraum towards the LEH. That scattered light can be collisionally absorbed and then heat the plasma in the LEH, thus changing the plasma conditions that can then affect the crossbeam transfer!

\subsubsection{Status of theory and modeling}

From the earliest days of the laser fusion program, the need to understand and control undesired LPI was recognized. The time and spatial scales of plasma formation in and around the hohlraum; the onset of SRS, two plasmon decay, filamentation and SBS; and wave particle interactions that generate hot electrons are so different that a single approach is not suitable to model all three.

Laser and plasma conditions are modeled on the macro scale within the radiation hydrodynamics codes such as 2D LASNEX [2] and 3D HYDRA [3].

Modeling the complexities of non-LTE states of the partially ionized high-Z ions in the plasma is an important component in how the rad-hydro codes calculate the radiative rates of energy loss from the 
plasma. Currently, either an average atom XSN nonLTE model [4], or a more sophisticated Detailed Configuration Accounting (DCA) non-LTE model [5] is used. Electron heat conduction is "flux limited" to ensure reasonable answers in the presence of thermal gradient scale lengths shorter than an electron mean free path. Packages that approximate this inherently "non-local" situation [6] are also implemented into the codes [7]. The best available current model [8] uses DCA that includes dielectronic processes, as well as a non-local transport model. These two components tend to predict higher radiative and electron thermal heat flux than the previous model. Hence, it is called the "high flux model" (HFM).

SRS, filamentation, and SBS can be modeled on a variety of levels of complexity. Currently on the meso scale, these processes are modeled with pf3D [9], which includes the laser propagation (and the subsequent scattering) in the paraxial wave approximation. It currently "post-processes" a piece of the plasma ( 10 cubic $\mathrm{mm})$ for a portion of the time $(\sim 100$ psec). Particle-in-Cell (PIC) codes [10] and kinetic codes study these phenomena on the micro scale, while including sophisticated models for the non-linear dynamics.

CBET and SBS are not well-understood at a detailed level. As with SRS, kinetic modeling of SBS (e.g., with a Vlasov or particle in cell (PIC) simulation augmented by collisional physics) is computationally challenging. A 3D kinetic model of SBS over the pulse lifetime in the complex target geometry is not expected in the near future. There is some evidence that CBET decreases during the pulse, in which case its saturation mechanism should be understood. A combination of codes, SLIP [11] and hydro codes calculate the level of CBET. Spatial inhomogeneity of the post CBET laser beams can affect $\mathrm{x}$-ray production, symmetry, and the downstream LPI. In addition, there is an often seen phenomenon in which SBS competes with SRS.

\subsubsection{Impact of experimental results}

What has been discovered, mainly on the basis of the 2009 energetics campaign on NIC, is that the hohlraum plasma conditions are different than our initial expectations, which were based on older, simpler models. The HFM tends to predict higher radiative and electron thermal heat flux than the previous model. The implication of these two higher fluxes (and especially as they act in concert with each other) is a prediction of a decidedly cooler hohlraum plasma. This cooler plasma is consistent with the SRS shorter wavelength than expected spectra vs. time [12] and its larger than expected amount of scattering, as well as with the capsule implosion symmetry [13], as computed taking into account beam propagation through that cooler plasma. New hohlraum shapes [14] were instituted based on this model that have proved efficacious in controlling P2 (second-order Legendre polynomial) symmetry in subsequent shots. Thus, the impact of this change in our expected plasma conditions has been very large. While this is a "feel good story" of better physics approximations making for better answers, it should be recognized that while data consistency is fine, until actual direct measurements of the plasma conditions are made, the HFM's accuracy cannot be assured.

The current ignition-scale experiments have a coupling efficiency of about $84 \%$. About $3 \%$ losses are due to SBS, while the bulk of the loss is due to SRS and primarily on the NIF inner beams. The SRS process also typically produces [15] about $100 \mathrm{~kJ}$ of hot electrons with a distribution that can be characterized by a temperature of $\sim 20 \mathrm{keV}$. In addition, about $1 \mathrm{~kJ}$ of $80 \mathrm{keV}$ hot electrons is observed, whose origin may be owing to phenomena at a quarter critical density such as Raman forward scattering or $2 \omega_{\mathrm{p}}$ instabilities.

CBET is required [16] in current NIC hohlraums to ensure a symmetric image of the hotspot upon implosion. Roughly $1 / 3$ of the outer beam power (which originally contained $\sim 2 / 3$ of the total power) is transferred to the inner beams. Thus, since the losses are mostly from these post-CBET inner beams, about $4 / 9$ of the total power ends up on the outer beams. About an equal amount ends up on the inner beams, the balance being lost back out of the hohlraum, due to SRS from those inner beams. There is evidence that the CBET process proceeds in an unsaturated mode early in the pulse, and eventually saturates and lessens later. This too must be experimentally characterized more directly. Moreover, the CBET is currently modeled as spatially uniform from and to each beam. Clearly, the post CBET laser beam spatial non-uniformity must be modeled and measured much better than is currently done.

Finally, it should be mentioned that capsule performance issues might have their origin in LPI processes. The apparent "stall out" in capsule drive 
during the rise of the main pulse may be due to internal LPI processes that redistribute light (at least temporarily) into parts of the plasma (e.g., low-Z gas fill) that are less effective in producing $X$-ray drive. Currently, the hydro codes or pf3D do not model processes such as Raman or Brillouin side scatter that would be candidates for this effect. The dense part of the ablator is observed to be decompressed more than the model predictions. This may be due to the hot electrons. There is also the possibility that moderate mode-number asymmetries (e.g., P4, P6, M4,...) that may be present in the dense shell surrounding the hotspot might have their origin in LPI processes. These modes, interacting with otherwise nominal mix, may account for low yields. With all of the abovementioned impact of LPI on experimental results, there is clearly a need for serious efforts in LPI research.

\subsection{Opportunities for Progress}

There are uncertainties in the laser and plasma conditions that control LPI, CBET, and beam propagation. Since non-LTE implies that local collisional rates do not entirely dominate, this means that the radiation field, in part, determines radiative rates, and does so via non-local influence. This is a very difficult, self-consistency challenge. Similarly challenging is the non-local thermal electron heat transport, wherein electrons with mean free paths much longer than a local thermal gradient contribute to the heat transfer. All of these determine the local plasma conditions and the nature of and level of the LPI processes that can ensue. The large scale-length of plasma at the ignition scale magnifies the challenge, as formerly insignificant optical depths for processes in plasmas now can contribute in a substantial way.

The SRS process makes hot electrons. They are currently modeled as diffusing isotropically from their point of origin within the hohlraum. To the degree that they act in a more beam-like mode of transport, then modeling them as beaming along the direction of the electron plasma wave may change how they affect hohlraum energetics and direct capsule coupling. As the role of hot electrons introduces an uncertainty to our understanding of capsule performance, it would be quite opportunistic to design experiments that minimize their creation. This could include achieving symmetry by beam balance alone, without utilizing CBET, which, after all, maximizes the inner beam intensity from which the SRS is emerging. Another technique would be to promote SBS as a way to depress the hot electron producing SRS.

Since SRS dominates the loss mechanisms to date at NIC, there is a need to develop a better understanding of the fundamental nature of the SRS process. This could allow us to accurately assess the onset of SRS and its saturation levels and then move on to mitigation strategies, either by means of changed plasma conditions or by means of changed laser driver, including a variety of wavelength, bandwidth, and modern laser pulse shape modulation approaches.

The role of ponderomotive and thermal filamentation in creating hotspots leading to magnetic field generation and enhancing intensities above the thresholds for SRS and SBS is not fully understood within the hohlraum. The parametric processes that take place at the critical surface, such as the parametric decay instability and oscillating twostream instability that can produce fast electrons, also need to be quantified.

The possibility that SBS will create significant loss of hohlraum coupling and capsule drive symmetry as the incident laser drive energy is increased cannot be excluded. It is, thus, important to develop an understanding of SBS and to explore and have ready concepts that can potentially, if needed, reduce its impact. This can be through new experiments that explore aspects of the physics and new concepts for LPI control and mitigation and the theoretical and numerical study of these ideas. Again, fundamental understanding of LPI onset and of the various competing saturation mechanisms would be important elements of this understanding.

The CBET is related to ion wave science and can benefit from such advances in fundamental understanding. It appears that CBET possibly self saturates in time, within a given experiment. Thus, understanding that process in detail will help us control symmetry in the NIC in a precise time dependent way. It may be imperative to do so, if indeed, shell asymmetries are currently coupling to ordinary mix in the present NIC experiments leading to low yield.

Moreover, the time dependent CBET also informs what the time and spatial dependent laser beam profiles are and where and how much SRS occurs. These can all affect internal LPI (processes that do not 
reveal themselves as readily as backscatter, because they do not emit their characteristic scattered light outside the hohlraum). This internal LPI could be affecting the lack of effective drive during the rise of the main pulse. Obviously devising ways to diagnose if such processes are happening is an important opportunity to resolve this key uncertainty.

A grand calculational challenge is to link the macro-scale hydro codes to the micro-scale PIC and kinetic codes, possibly by way of the meso-scale codes, by implementing within them reduced model descriptions of the kinetics. Another opportunity would be the less grandiose, but still grand challenge of definitively converging a full rad hydro calculation- zoning, photon bin, and electron bin. Part of this grand challenge is to accurately model not only the real world complexities of the actual target construction and geometry, especially the complexities surrounding the LEH, but also including diagnostic holes, the tent that suspends the capsule within the hohlraum, and the movement outward of the outside of the hohlraum wall.

To match capsule implosion dynamics, target designers and experimental analysts must currently multiply the peak laser power by a factor less than one. Yet it is the nominal calculation, without multipliers, that "matches" the Dante flux that emerges from the LEH. This points to the possibility that there are cancelling errors in the modeling of the $\mathrm{x}$-ray production and of the LEH size. A better full rad-hydro simulation, with better-resolved zoning of the laser-to- $\mathrm{x}$-ray conversion layer may lower the predicted drive. That could well go hand in hand with better resolved LEH dynamics, and the two cancelling errors could turn into two well-predicted phenomena that together can "really" match the Dante flux and get the right answer for the right reasons.

Another issue in modeling drive is the need to pick a particular condition (usually on Te) at which to switch from the non-LTE modeling to an LTE table of data for opacity and equation of state (EOS). The presumption here is that the tables give reasonably accurate answers that have been validated, to some degree, by experiments. The calculated drive seen by the capsule is sensitive to the choice of what $\mathrm{T}_{\mathrm{e}}$ at which to "turn on the switch". Thus, the choice of that "switch" condition adds uncertainty to the calculation. Having a non-LTE model that naturally switches to LTE in a way that gives answers very close to those of the tables would eliminate this uncertainty.

Since the PIC codes deal on the most fundamental of levels within our reach today, and on the shortest temporal and spatial scales, it also behooves us to develop experimental diagnostics and platforms that can confirm some of the kinetic physics on those short temporal and spatial scales, studying single speckle systems, and then go on to learn how ignition-scale relevant multi-speckle systems interact and behave.

\subsection{Priority Research Directions}

\subsubsection{Hohlraum Plasma Characterization}

\section{Introduction}

Both laser and plasma conditions control LPI, CBET, and beam propagation. Modeling the complexities of non-LTE states of the partially ionized high- $\mathrm{Z}$ ions in the plasma is an important component in how the rad-hydro codes calculate the radiative rates of energy loss from the plasma. Currently, either an average atom XSN non-LTE model or a more sophisticated DCA non-LTE model that includes dielectronic recombination is used. A "non-local" heat transport model in combination with DCA predicts considerably higher flux of heat and radiation than previous simpler models. It constitutes the new "standard" high flux model, HFM.

The HFM predicts a cooler plasma in the NIC hohlraum than that predicted by XSN. It is imperative that this (new standard) model is verified by direct measurements of the plasma conditions. It would be unwise to proceed to doing the rest of the science of ignition without being firmly "grounded" in knowing the plasma and laser conditions.

\section{Near-term improvements and approaches to theory and modeling}

The modeling of non-LTE plasmas could be improved to ensure that in the LTE limit it can reproduce the results of the very best atomic physics codes available. Advanced kinetic treatments of heat transport (e.g., Fokker Planck) can be applied, at least to low-Z plasmas. On what may seem to be a more mundane plane, what is needed are heroic efforts at making the very best hohlraum model achievable, in terms of zoning and photon bin and electron bin convergence and that includes a great deal of real 
world structures within and outside the actual hohlraum.

Since magnetic fields can affect electron conduction and thus plasma conditions, efforts should be made that ensure that all relevant $B$ field generation terms (e.g., Nernst terms) are properly implemented into the rad hydro codes. To date this has proven to be very challenging.

On a shorter time scale, there is a need to complete the process of implementing into the hydro codes the more self-consistent models that allow for SRS to occur within the hohlraum plasma and send its scattered light back, possibly within a broader angular cone, through the plasma, thus heating itself consistently. Similarly, codes that compute in situ the CBET (as well as its saturation mechanisms) should be implemented. These affect the CBET vs. time, as well as the post CBET laser intensity spatially nonuniform profile. Both aspects may affect the conversion of laser light to X-rays, as well as the symmetry and downstream LPI.

High impact experiments on HED facilities to address uncertainties in critical physics models

There is a need for measurements of $\mathrm{T}_{\mathrm{e}}, \mathrm{T}_{\mathrm{i}}, \mathrm{n}_{\mathrm{e}}, \mathrm{Z}$ and $\mathrm{v}$ in at least four places: the LEH, in the gold blow-off, in the gas volume from where the SRS emerges, and finally, above the waist of the capsule, at the hohlraum mid-plane. The measurements might be Thomson scatter, using either $4 \omega$ light, or possibly cheaper but with compromises, using $3 \omega$ or even $2 \omega$, where $\omega$ is the fundamental frequency of the laser light. Alternative methods such as "dot" spectroscopy should be assessed. If possible, either of these methods might also look for B fields that arise within the hohlraum or at the LEH. Knowing the plasma conditions will greatly amplify the efficacy of studies that re-optimize hohlraum performance by changing gas fill density and composition, including perhaps foams that can break LPI coherence.

Since the test bed for the HFM was the Au sphere experiments [17] on the University of Rochester Laboratory for Laser Energetics (LLE) OMEGA laser, efforts should be made to improve upon them. The absorption fraction should be measured separately, and through two-color Thomson scatter, the $\mathrm{T}_{\mathrm{e}}$ and densities in the gold blow-off should also be measured. These new data will provide a far more stringent test of the HFM.
The highest priority research direction is: Increase $\mathrm{T}_{\mathrm{e}}$. This could reduce SRS (and possibly SBS and CBET too, if so desired), increasing coupling efficiency. With a hotter plasma, beam propagation would be better and reduce even the need for CBET. To be cautious (and humble), the LPI may nonetheless "find a way" under these conditions to be troublesome anyway - but that only makes the prospect of such an experiment more interesting! $T_{e}$ could be made hotter by doping the hohlraum interior with higher-Z materials, introduced from either the outside of the ablator, in some of the aforementioned foam fillings, or to some degree, within the fill gas. $\mathrm{T}_{\mathrm{e}}$ can be increased by scaling the hohlraum size down. A change in hohlrum geometry (such as a "rugby" [18]) might allow better beam propagation and thus a higher $\mathrm{T}_{\mathrm{e}}$. Another approach, which should be tested first at the OMEGA laser, is to impose an external B field on the hohlraum to help reduce thermal conduction. This may have another benefit of insulating the capsule from hot electrons. As will be discussed in Sections 1.5 and 1.6 , if LPI can be mitigated by the means described therein, coupling will improve and $\mathrm{T}_{e}$ will rise.

New capabilities (diagnostics, models) needed

Using Thomson scattering (TS) is a well-known method of determining many key plasma condition parameters. It is perhaps possible when diagnosing the region near the LEH to get by via "cheaper means" than $4 \omega$ TS, namely $3 \omega$ or $2 \omega$. Near the LEH proton beam, probing may be feasible. Using "dot spectroscopy" to detect plasma conditions is another possible method. This has been utilized in the past in open geometries and to a lesser degree within hohlraums.

Dedicated diagnostics that can monitor the time dependent closure (at various relevant $\mathrm{X}$-ray frequencies) of the LEH would be extremely valuable. In a similar vein, instruments that monitor the time dependence of the outside of the hohlraum wall's expansion would be an excellent constraint on the modeling and inform issues of increased wall loss due to larger wall area, especially at late times. Monitoring the drive not through the usual Dante line of sight, but through a line of sight more directly relevant to the capsule view would also be a valuable experiment.

Varying plasma fill gas pressures, gas materials, or filling types (e.g., foams), as well as scaling plasma 
and laser power sizes etc., are all ways to vary plasma conditions, assess LPI, etc. The diagnostic techniques described above would then be exercised in significant ways.

Utilizing advanced models that can sensitively predict plasma conditions is another important aspect to this problem. Examples of such needed models include: advanced Fokker Planck kinetic transport codes; models that can be implemented into the hydro codes that account for saturation mechanisms of LPI and CBET that involve, for example, ion heating; and non-LTE models for high- $Z$ ions that can achieve, in the LTE limit, the same results for opacity and EOS as those of the most sophisticated LTE tables.

\section{Long-term goals and outlook}

A very challenging "stretch goal" would be to incorporate the non-Maxwellian distributions that emerge from such kinetic treatments into atomic physics non-LTE codes that would respond to such distributions, e.g., vis a vis collisional rates. Another challenge is to implement models that can deal with interpenetrating plasmas, as well as Au-gas interface mixing in general.

\subsubsection{Electron Plasma Wave Science}

\section{Introduction}

The Raman process is currently the chief actor in the coupling losses from ignition-scale hohlraums, especially from the inner beams. The SRS saturation levels, how and why SBS competes and "turns off" the SRS, and the subsequent production of possibly deleterious hot electrons are all major high-leverage issues, that require better understanding if progress is to be made in mitigating this effect.

The current levels of SRS are of sufficient magnitude to make this a priority area of research. As the ignition research proceeds to thicker ablators on its ICF capsules, more drive energy will be required to get those capsules up to the required implosion velocities. Increasing coupling by decreasing SRS will be very helpful in that endeavor.

\section{Near-term improvements and approaches to theory and modeling}

On a fundamental level, cross code comparisons should be made to maximize the lessons learned from the fundamental PIC and kinetic studies. These can be of value from 1D comparisons up through 3D. Interesting work on the interplay of non-local heat transport and SRS via growth rates modified by the Landau Damping changes due to the heat flow should be pursued.

We must complete the process of implementing into hydro codes the more self-consistent models that allow for SRS to occur within the hohlraum plasma and send its scattered light back through the plasma, thus heating the hohlraum plasma self-consistently. Improvements beyond what might be accomplished in the near term could include propagating the backscattered light with gain and not simply creating a fixed amount of scatter at some given point in space.

We should validate, through test problems and comparison to specifically designed experiments, the current capabilities of the rad hydro codes to properly account for the atomic physics and ensuing radiation caused by hot electrons impacting inner shell processes in the ions of the high- $Z$ walls of the hohlraums.

High impact experiments on HED facilities to address uncertainties in critical physics models

Small-scale experiments that can monitor the very basic Raman physics processes on the very short time scales of the SRS process would be a valuable exercise of the science and of the PIC codes that describe it. They will require diagnostics with very good time resolution (1 to $10 \mathrm{psec}$ ). Larger-scale experiments can then monitor multi-speckle-size plasmas and the effects thereof. Assessing fundamentals such as SRS decreasing via wave breaking and turning on and off counter propagating beams to assess SRS transmission should be considered.

The production of hot electrons can result in direct preheat of the target. While experiments to date have attempted to quantify that effect, continuing vigilance at monitoring the capsule preheat directly is recommended, especially as the ignition research program proceeds to longer pulses with more power and energy. Methods to monitor the higher energy $x-$ rays (the $M$ band) that can radiatively preheat the capsule should also be developed to address the following issue: Dante sees M band coming out of the $\mathrm{LEH}$, which may not be equal to the $\mathrm{M}$ band incident onto the capsule. 
Experiments that optimize the hohlraum fill can be done by varying the fill gas pressure, its elemental make-up, and even the hohlraum geometry. Fill strategies that include structures, especially meant to break up coherence and reduce SRS, such as foams or micro-needles, should be examined. Foams that vary spatially in their composition ("zebra foams") might breakup coherence as well. As mentioned in Section 1.4.2, increasing $\mathrm{T}_{\mathrm{e}}$ by a variety of means may directly reduce SRS. Again, as mentioned above, imposing an external B field on the hohlraum to help reduce thermal conduction is a particularly interesting method of raising Te.

Even if experiments do not result in increased coupling, but simply reduce SRS, they would be valuable as they could eliminate questions of hot electron effects. One approach would be to design a hohlraum that favors SBS, which competes and beats down the SRS. Other efforts that minimize CBET could reduce the inner beam intensity and reduce the level of SRS.

The previous paragraphs addressed reducing the SRS by plasma and/or competitive LPI means. An entirely different approach involves reducing LPI in general by laser means. From the macro scale, one can consider using $4 \omega$ light on the inner beams to reduce SRS. Issues of whether the $4 \omega$ will beat with the $3 \omega$ beams and serve as enhanced noise for other LPI processes should be assessed. Conversely, $2 \omega$ could be explored as it allows for more drive energy. Other laser mitigation methods involve scaling studies of bandwidth, and/or spatial incoherence, temporal incoherence (smoothing by spectral dispersion [SSD]), and/or polarization methods. All are worthy of dedicated experiments.

A particularly exciting laser approach to LPI mitigation is to attack the coherence on a very fundamental instability time scale. These rapid and adaptive amplitude modulated pulses, such as "spike train of uneven duration and delay" ("STUD" pulses ${ }^{19}$ ) should be assessed on the appropriate laser facilities. Experiments with $10 \mathrm{ps}$ time scale modulations could be tested at OMEGA (URLLE) or Trident (LANL). The more ambitious program of operating down at the 1-ps time scale would have to wait for hardware changes not currently anticipated at NIF. Such pulses could disrupt SRS via halted growth spurts and spatial scrambling of active hotspots in between laser spikes.

\section{New capabilities needed}

The early time hot electron preheat of the core is thought not to be a problem as the currently used, broad-band filter-fluorescer hard x-ray "FFLEX" diagnostic shows hard x-rays above $170 \mathrm{keV}$ only after the onset of the $4^{\text {th }}$ picket. Again, higher power shots planned with a thicker plastic ablator could generate significantly more hot electrons that could make preheat an issue again. It would be better to directly measure the hot electron energy deposition at the core of the capsule using perhaps a high- $Z$ emitter placed at the core of a capsule. This possibility should be explored or revisited given the importance of ruling out preheat as a factor leading to an increase in entropy of the fuel core. Having more channels of FFLEX time resolved would also be of value as it could be correlated with the observed temporal behavior of the SRS scattered light.

Developing diagnostics with excellent time resolution (1-10 ps) is another priority, as it will allow more fundamental experiments on SRS and its onset and saturation.

Since Raman side scatter is of some concern, efforts to invest in ways to expand the pf3D code to include non-paraxial effects should be undertaken.

\section{Long-term goals and outlook}

The grand computational challenge of LPI is to find ways to marry the multi-scale physics inherent in the process.

We currently model the hot electrons created by SRS as diffusing isotropically from their point of origin within the hohlraum. To the degree that they act in a more beam-like mode of transport, then modeling them as beaming along the direction of the electron plasma-wave may change how they affect hohlraum energetics and direct capsule coupling. As local electric and magnetic fields can affect such beaming trajectories, this is a very difficult computational challenge, somewhat akin to the (again multi-scale) transport problems encountered when studying fast ignition.

\subsubsection{Ion Wave Science}

\section{Introduction}

Ion plasma wave physics has at least two important impacts on NIC: the first is the positive impact of facilitating CBET. The second impact is 
made by SBS, which can limit laser beam coupling. With higher energy and longer pulses, there is a danger of increasing levels of SBS. Thus, similar to SRS, SBS too is priority area of research. Moreover, with the thicker ablators come more plasma in the hohlraum and longer pulses, thus further challenging the implosion symmetry. CBET may, therefore, continue to be a key tool in obtaining proper implosion symmetry. Thus, CBET is a key piece of science to better understand and control, and, because it is mediated by ion waves, they too are included here for discussion. The filamentation instability will create increased intensities for SBS to grow rapidly within the filament as well as producing $\mathrm{X}$-ray hotspots. The subsequent filamentation hotspots will produce magnetic field structures that will alter electron transport. LPI at the critical surface where the parametric decay takes place will alter laser absorption and the generation of fast electrons giving rise to non-Maxwellian distributions.

\section{Near-term improvements and approaches to theory and modeling}

Simulations and models could be crosschecked in other LPI physics experiments in many laboratories.
CBET and SBS are not well understood at a detailed level. As with SRS, kinetic modeling of SBS (e.g., with a Vlasov or PIC simulation, augmented by collisional physics) is computationally challenging.

There is some evidence that CBET decreases during the pulse, in which case its saturation mechanism should be understood. The current efforts at modeling the CBET saturation via ion kinetic selfheating should be pursued and implemented into the hydro codes, as should other more self-consistent modeling such as the in-line CBET itself. That model may show saturation due to ponderomotive forces and/or the evolution of the velocity field. Finally, the self-consistent SRS package should be included in the simulations, as the backscattered light can locally heat the LEH region and change the CBET.

High impact experiments on HED facilities to address uncertainties in critical physics models

Experiments of specific aspects of NIF LPI physics might be performed at OMEGA, and simulations and models could be crosschecked in other LPI physics experiments in many laboratories. The hohlraum can be modified (for example, using

\section{LPI and Beam Propagation in Hohlraums}

Laser beams propagate through the NIC hohlraum's laser entrance holes and proceed toward the interior gold walls that absorb that light and convert thermal energy to $\mathrm{x}$-rays used to drive the capsule. LPI might also occur along the way, such as SBS and SRS instabilities, which scatter the light off self-generated ion acoustic and electron plasma waves respectively. Loss of light out of the hohlraum due to LPI reduces capsule drive. The SRS electron plasma wave can also produce hot electrons that can preheat the capsule. In addition, LPI processes can re-direct the light, thus affecting the symmetry of the capsule drive.
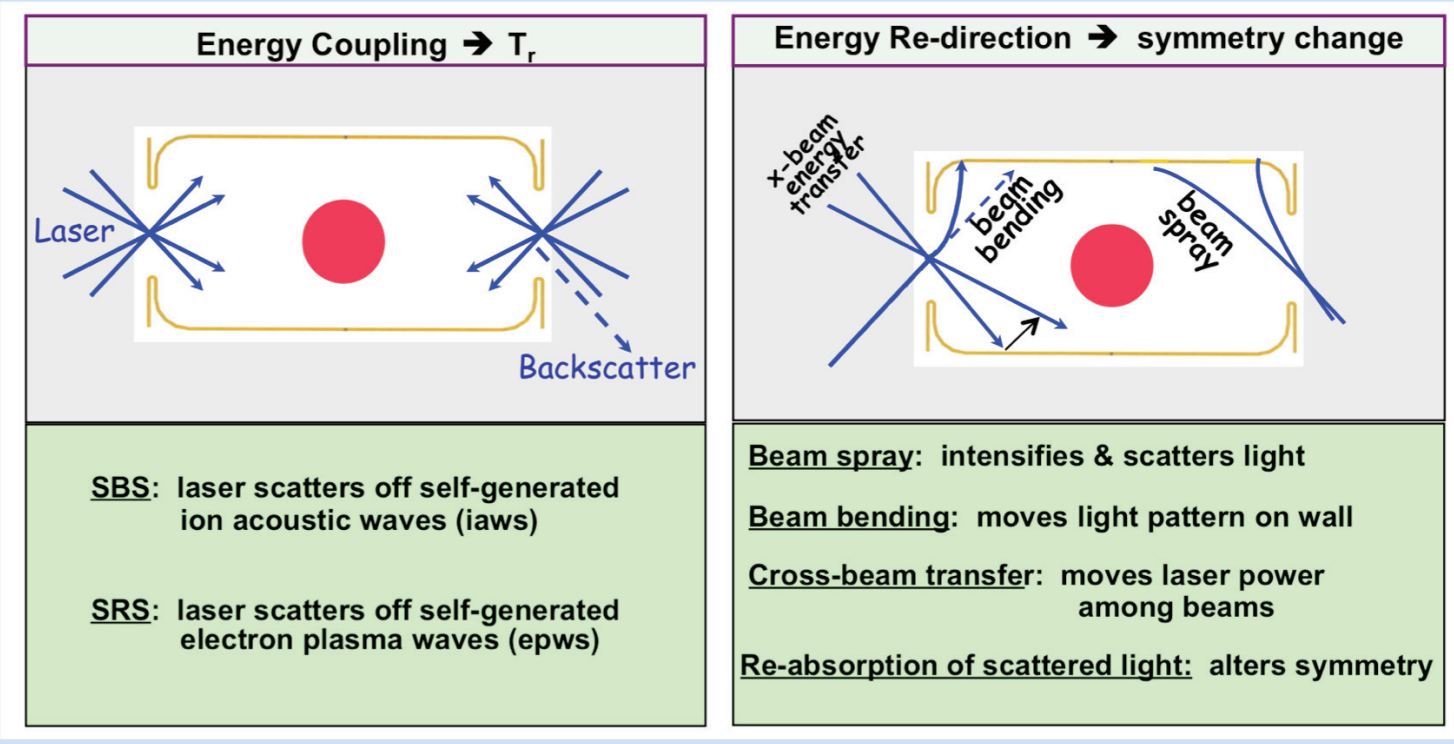

Panel 1 - Laser Propagation and X-ray Generation 
one half of a hohlraum) to allow for enhanced diagnostic access and elucidation of specific aspects of the pulse dynamics. Temporal control of the laser pulses for LPI studies may also be desired.

Given the difficulty in understanding, modeling and controlling the detailed plasma microphysics associated with cross beam transfer, it is desirable to investigate the feasibility of schemes that make CBET an optional, rather than a necessary, tool to achieve drive symmetry. Some ideas for achieving this goal include reducing SRS, thereby reducing the need for cross beam transfer. Furthermore, by means of temporal interlacing of drive pulses (STUD pulses), CBET could be controlled, and SRS may also be mitigated. These ideas are worth exploring theoretically and-as feasible-experimentally. One suggested method to "tame" SBS is to consider Au/B as a liner (in a DU hohlraum).
Aspects of ion wave physics can be elucidated via an SBS-dominated experiment that would enable us to separate the SBS and SRS effects. Another important experiment is to create a "semi-open" geometry that still acts as a good "LEH mock-up", but is optimized to monitor transmitted beam profiles. This platform could be tried first at OMEGA and then tried at NIF. Within such a platform we could vary and study CBET by varying plasma conditions, varying plasma composition, varying laser conditions, e.g., bandwidth or STUD like pulses, or some grand combinations of all of these techniques. Similarly efforts at designing full hohlraums but with "holes" in the wall ("holedraums") that allow monitoring the post-CBET beam profile, though a challenging design task, should be explored.

Another possibility is performing experiments at $1 \mathrm{MJ}$ drive on target with and without CBET (the experiments without CBET would use more intense

\section{Multi-scale Modeling of LPI in Hohlraums}

LPI processes are modeled on the macro, meso, and micro scale. It is a grand computational challenge to unify these levels of description, incorporating all the necessary physics at all relevant length and time scales. One approach to this goal would be first begin to merge the meso- and micro-scales by development of reduced models of kinetic effects within pf3D. One could also begin to merge the macro- and meso-scales by coupling pf3D to rad-hydro simulations on a very crude time scale. Increases in computing power make both of these approaches viable.
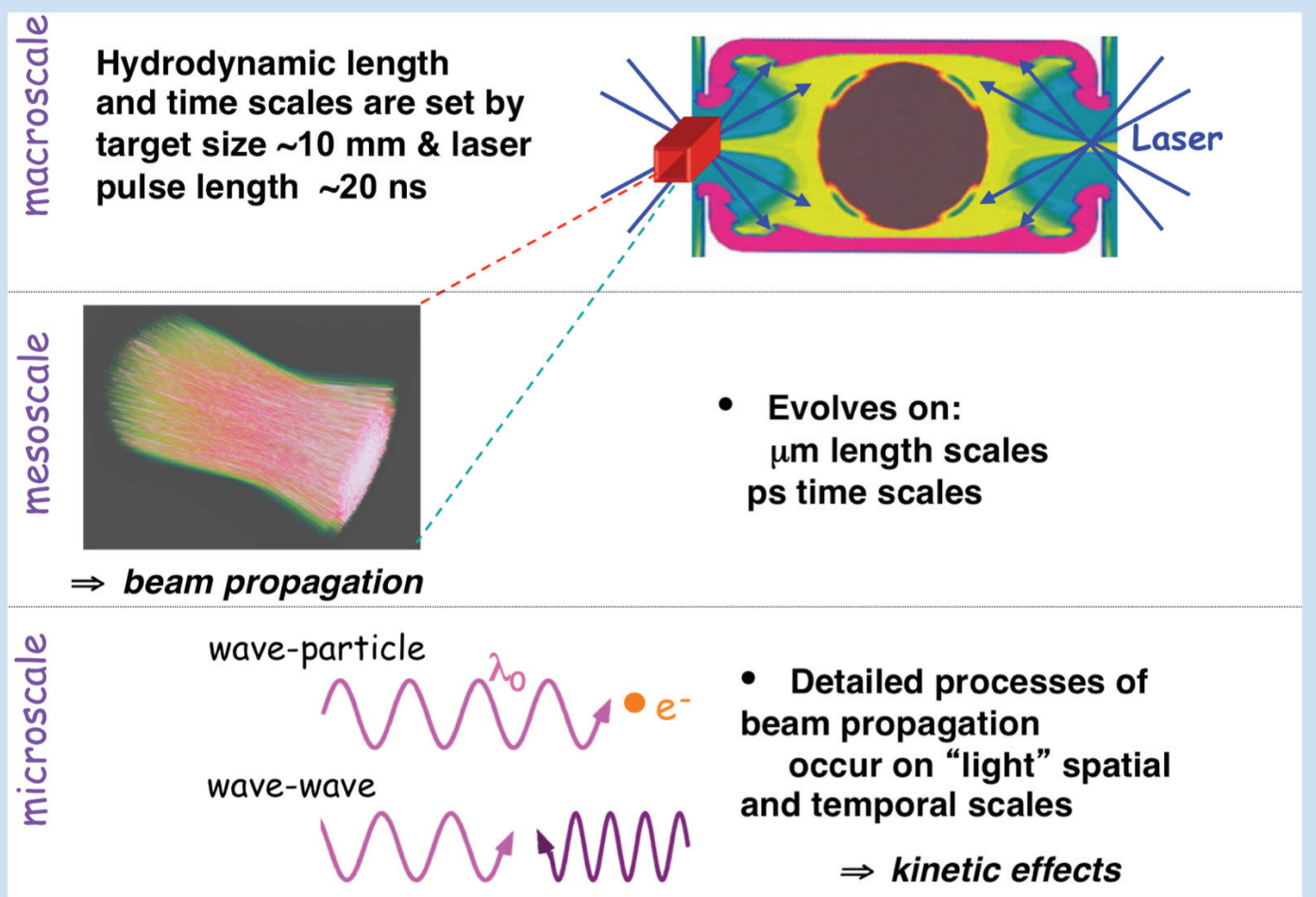

- Detailed processes of beam propagation occur on "light" spatial and temporal scales

$\Rightarrow$ kinetic effects 
inner beams). While current hydro models predict no difference between these two scenarios, this is precisely why it is an interesting experiment. It might show surprises that point to deficiencies in the current modeling. Another method to eliminate the need for CBET would be a change in hohlraum shape.

The current NIF pulses overlap in the LEH. At the expense of higher pulse intensity, the pulses could be temporally interwoven (STUD pulses). These pulses would be $\sim 10 \mathrm{ps}$. This geometry might allow for greater CBET control, and, by using shorter pulses, the Raman growth could be disrupted. At fixed energy, however, instabilities may have shorter growth times and, thus, the scaling of the number of e-foldings should be studied in detail.

\section{New capabilities needed}

Understanding ion wave physics is hampered by a lack of detailed information on the temporal evolution of the plasma. As mentioned earlier, a Thomson scatter diagnostic could be explored with the goal of measuring ion waves, $\mathrm{T}_{\mathrm{i}}$ and $\mathrm{T}_{\mathrm{e}}$, as functions of time.

Since stimulated Brillouin side scatter cannot be ruled out, the pf3D code should be expanded to allow for non-paraxial effects.

\section{Long-term goals and outlook}

A 3D kinetic model of SBS over the pulse lifetime

\section{Modeling Plasma Conditions in HohIraums}

The temperature, Te, and the density, $\mathrm{n}$, of the plasma that fills the hohlraum are important elements in determining the beam propagation in a NIC hohlraum. They affect laser beam absorption and the nature and level of LPI processes. Modeling heat transport is made more difficult by the presence of sharp temperature gradients, whose scale lengths are shorter than the mean free path, $\lambda$, of the electrons carrying the heat. Thus, describing heat flux by a classical Fick's law form of $F=-(n \lambda v / 3) d T / d x$ can lead to a non-physical flux that exceeds the free streaming value of nvT. A flux limiter, $\mathrm{f}$, must be applied to prevent this. It is a "free parameter" whose value has been traditionally changed to match data. In reality, the heat flux must be calculated as a non-local process. The modern hydro codes do have models for this non-local heat transport. In addition, $\mathrm{T}_{\mathrm{e}}$ is determined by the accuracy of the radiation emission rates of the hot gold plasma. More detailed models like DCA are modern improvements to the average atom XSN model. The figure shows how these modern tools affect the hydro code's predictions of the hohlraum's plasma conditions. The axis of rotational symmetry is the $\mathrm{x}$-axis, and the $\mathrm{y}$-axis is an axis of mirror symmetry. The capsule is in black.

\section{$\mathrm{T}_{\mathrm{e}}(0-5 \mathrm{keV}$ contours) in $1 \mathrm{MJ}$ hohlraum at $18 \mathrm{~ns}$ (middle of main pulse)}

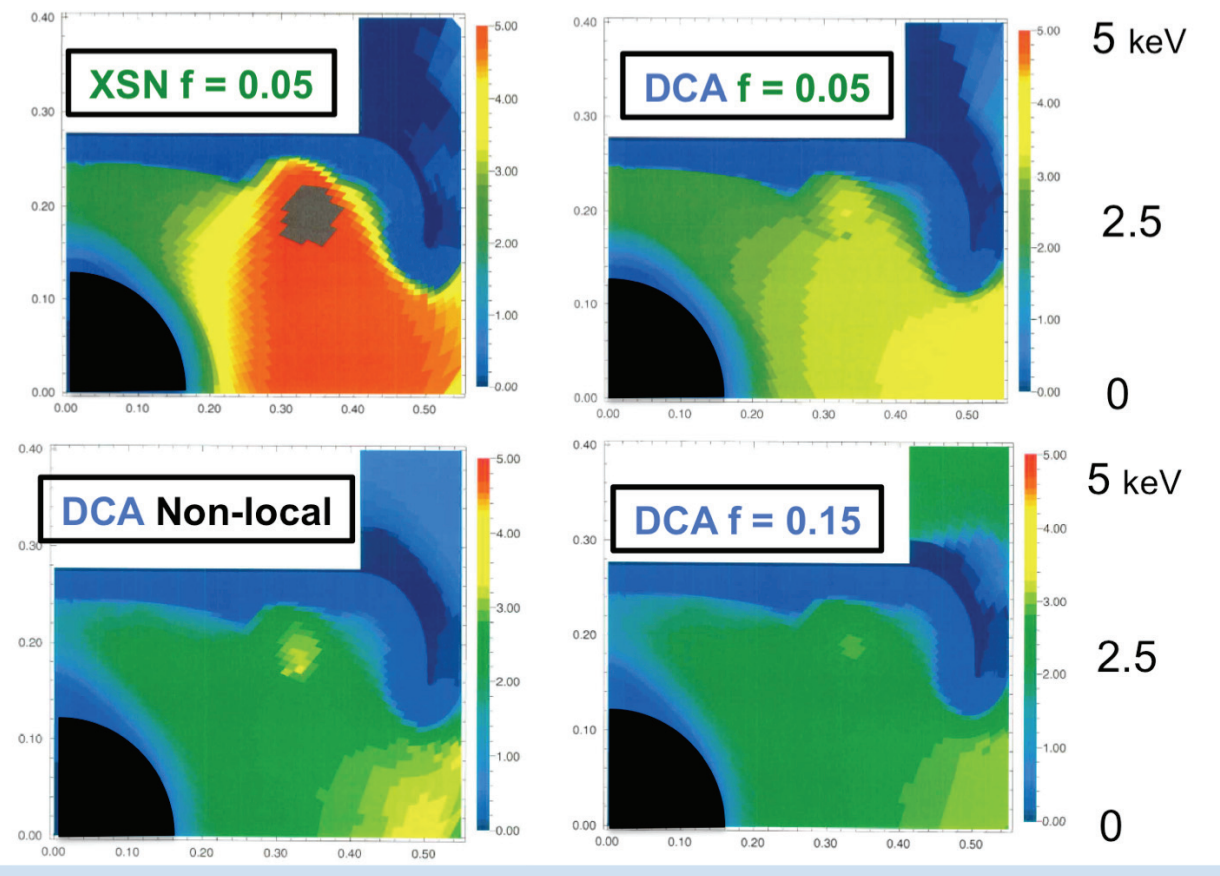


in the complex target geometry is not expected in the near future, but would be quite useful. Understanding how CBET proceeds under different ion wave damping conditions is an important step in establishing just how well CBET can be controlled.

A goal for diagnostics is a temporal resolution of LPI saturation processes $(\sim<10 \mathrm{ps})$.

The experimental and theoretical ideas laid out above and proposed to be tested on the appropriatescale facilities have high potential for leading the way to LPI mitigation and thus helping in the very difficult goal of achieving ignition in the laboratory.

\subsection{Conclusions}

The high-priority research directions described above are summarized below:

\section{1a) Measure and better predict plasma conditions:}

- Measure plasma conditions, such as $\mathrm{T}_{\mathrm{e}}, \mathrm{T}_{\mathrm{i}}, \mathrm{n}_{\mathrm{e}}, \mathrm{B}$ fields, in key parts of the hohlraum, especially near the LEH. Methods may include Thomson scattering or spectroscopy. This is the basis for proceeding with further study of all of the other LPI and x-ray production science initiatives.

- Redo the OMEGA Au sphere experiment with more diagnostics such as absorption fraction and $\mathrm{T}_{\mathrm{e}}$ via two-color Thomson scattering. This will more tightly constrain the High Flux Model.

- Improve macro packages in the rad-hydro codes such as SRS internal to the hohlraum and a CBET in-line model. Do "hero" calculations of a full hohlraum with all the details of the actual LEH, target holder, converged zoning, full B field terms, etc. Diagnose the LEH closure and the outer hohlraum wall expansion.

- Explore interpenetrating plasma models. Improve more fundamental micro packages such as kinetic/Fokker Plank head transport. Improve nonLTE DCA so that it matches STA-Vista opacities in the LTE limit.

\section{Continuous Improvements to Modeling Plasma Conditions in Hohlraums}

Efforts are ongoing to continually improve modeling of the NIC hohlraum so that it better reflects, with greater fidelity, the actual ongoing physics in the hohlraum. In the figure we see how increasing the quality of the model (flux limit upgraded to a non-local package) changes the predicted plasma conditions in the hohlraum's laser entrance hole (LEH). A further improvement in the modeling allows for SRS to occur within the volume of the hohlraum. Previously, the SRS back-scattered light was subtracted from the incident beam even before it entered the problem. The back-scattered green light propagates back through the hohlraum and out the LEH. In that process, some of the green light is absorbed and thus heats the LEH plasma even further. This might have the effect of changing the crossbeam energy transfer (CBET) there due to the changing plasma condition. Future models will include the CBET as an in-situ, in-line process and also include saturation models due to ion heating. This saturation and the concomitant change in Ti will both directly and indirectly (via changed plasma conditions) respectively, change the amount of CBET.

\section{$\mathrm{T}_{\mathrm{e}}(0-3.5 \mathrm{keV}$ contours) in $1 \mathrm{MJ}$ hohlraum at $18 \mathrm{~ns}$ (middle of main pulse)}
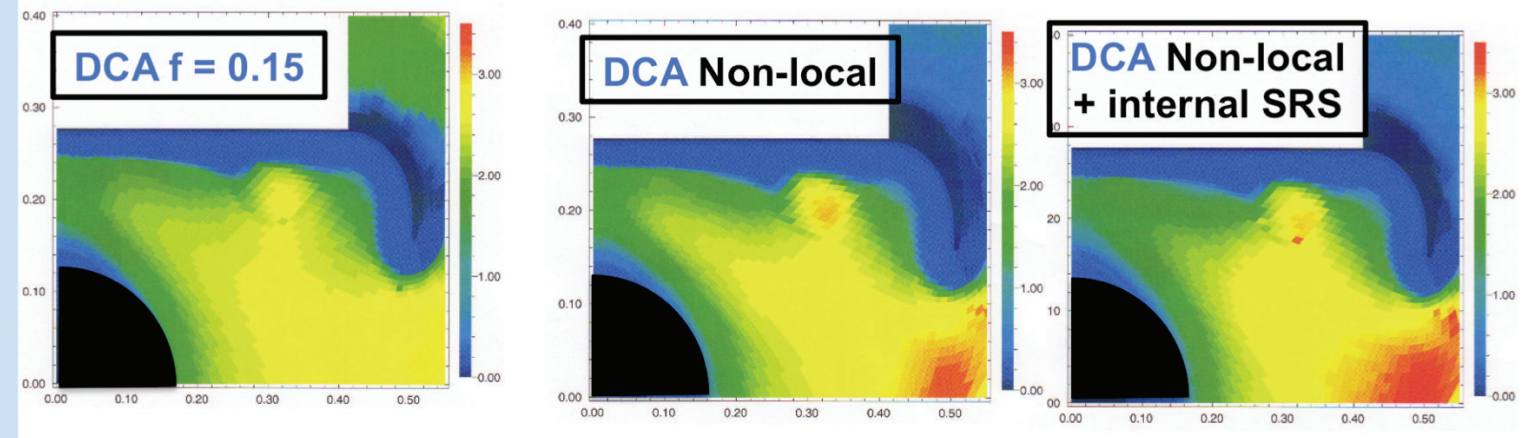


\section{1b) Improve Plasma Conditions:}

- Raise $\mathrm{T}_{\mathrm{e}}$ to quite possibly mitigate LPI and improve beam propagation. Methods may include imposed B fields, artificially raising the $\mathrm{Z}$ of the hohlraum fill, or hohlraum size and shape scaling. Verify the higher $\mathrm{T}_{\mathrm{e}}$ via the methods of \#1a above.

2) Understand Electron Plasma Wave Phenomena \& mitigate SRS:

- Expand our modeling capabilities from testing the basic PIC level through broadening pf3D capabilities such as allowing for non-paraxial effects and implementing reduced kinetic descriptions. Test these models using experiments with ultrafast diagnostics.

- Improve the hot electron transport package and its effects on atomic physics.

- Mitigate SRS by plasma means, such as foamfilled hohlraums that interfere with coherence or by promoting SBS to compete with SRS. This will allow monitoring target performance in the absence of hot electrons.

- Mitigate SRS by laser means, such as Bandwidth or STUD pulses.

3) Understand Ion Wave Phenomena \& mitigate SBS \& control CBET:

- Measure the non-uniform laser profile in its post CBET state by designing and deploying a semiopen geometry that mimics the LEH and its CBET conditions.

- Use that platform to study laser and plasma methods of controlling CBET.

- Compare capsule-hohlraum performance of two notionally equivalent situations: utilize CBET, or use an equivalent beam balance without CBET.

- Develop a CBET kinetic saturation model.

- Mitigate SBS by plasma means, such as Au/Blined hohlraums.

- Mitigate SBS by laser means, such as bandwidth or STUD pulses.

\subsection{References}

1. W. L. Kruer, "The Physics of Laser Plasma Interactions" (Addison-Wesley, Redwood City, CA 1988)

2. G. B. Zimmerman and W. L. Kruer, Comm. Plasma Phys. Control. Fusion 2, 51 (1975)

3. M. M. Marinak, G. D. Kerbel, N. A. Gentile, et al., Phys. of Plasma 8, 2275, (2001)

4. See National Technical Information Service Document No. UCRL52276 (W. A. Lokke and W. H. Grasberger, Report No. UCRL-52276, 1977). Copies may be ordered from the National Technical Information Service, Springfield, VA. Standard model using this is described in: M. D. Rosen, D. Phillion, V. C. Rupert, et al., Phys. Fluids 22, 2020 (1979).

5. H. Scott and S. Hansen, High Energy Density Physics 6, 39 (2010).

6. D. Shvarts, J. Delettrez, R. L. McRory, et al., Phys. Rev. Lett, 47, 247 (1981)

7. G. Schurtz, X. Nicolai and M. Busquet, Phys. of Plasma 7, 4238 (2000)

8. M. D. Rosen, H. A. Scott, D. E. Hinkel, et al., High Energy Density Physics 7, 180 (2011)

9. D. E. Hinkel, D. A. Callahan, A. B. Langdon, et al., Phys. of Plasma 15, 056314 (2008)

10. L. Yin, B. J. Albright, H. A. Rose et al., Phys. of Plasma 19, 056304 (2012)

11. P. Michel, L. Divol, E. A. Williams et al., Phys. Rev. Lett. 102, 025004 (2009).

12. D. E. Hinkel, M. D. Rosen, E. A. Williams, et al., Physics of Plasmas 18, 056312 (2011)

13. R. P. J. Town, M. D. Rosen, P. Michel, et al., Physics of Plasmas 18, 056302 (2011)

14. D. A. Callahan, N. B. Meezan, S. H. Glenzer, et al., Physics of Plasmas 19, 056305 (2012)

15. T. Doppner, C. A. Thomas, L. Divol et al. Phys. Rev. Lett., 108, 135006 (2012)

16. P. Michel, S. H. Glenzer, L. Divol, et al., Phys of Plasmas 17, 056305 (2010)

17. E. L. Dewald, M. D. Rosen, S. Glenzer, et al., Phys. of Plasma 15, 072706 (2008).

18. M. Vandenboomgaerde, J. Bastiani, A. Casner, et al., Phys. Rev. Lett. 99, 065004 (2007)

19. B. Afeyan and S. Huller, Europ. Phys. J. Web of Conf. (IFSA 2011, Bordeaux). 


\subsection{PANEL 2 - X-RAY TRANSPORT AND ABLATION PHYSICS}

\subsection{Introduction}

This section considers how the $\mathrm{x}$-ray drive generated in the hohlraum is transported and coupled to the ablator, generating the hydrodynamic drive that implodes the capsule. This coupling determines the implosion velocity, which must be sufficiently high to heat the deuterium-tritium (DT) "hotspot" to fusion temperatures as well as strongly compress the surrounding cold DT fuel. The coupling produces the multiple shocks that are launched into the capsule, adjusted in strength and timing to keep the fuel at the low entropy that is required to produce the necessary cold fuel areal density surrounding the hotspot. The issues described in this section concern what could be called the "1D" physics. In the ICF context, "1D" refers to the time-dependent radial profiles of density, pressure, temperature, velocity, etc., calculated for spherically symmetric implosions, since the capsule must remain close to spherically symmetric for most of its trajectory. The 1D dynamics are determined by the material properties such as equation of state (EOS) and opacity that are folded into the solution of the radiation-hydrodynamics equations together with the time- and frequency-dependent incident $x$-ray spectrum. Accurate 1D profiles are the essential starting point for modeling and interpreting experimental deviations from perfect symmetry that are thought to set the ultimate limits on performance of the capsule in the form of either long scale-length drive asymmetry or short scalelength instability. It is essential to understand whether the $1 \mathrm{D}$ physics is being modeled correctly before trying to understand higher dimensional effects such as mix of the cold fuel or ablator into the hotspot. Instability growth, in particular, depends on the details of acceleration history and density profiles. Enhanced "seeding" of instability or asymmetry can be tied to ablator and drive physics and is touched on in this section.

The primary issue related to the transport and coupling of the $\mathrm{x}$-ray drive to the ablator is that the experimentally observed implosion velocities are lower than predicted by the baseline 1D simulations. The estimated remaining mass is close to being consistent with the measured implosion velocity, suggesting that the "rocket" model is performing nearly as expected, though the remaining mass is higher than predicted in baseline simulations. Either the radiation drive is lower than predicted, or the ablator is less efficient at absorbing and converting the energy to useful hydrodynamic work than expected. The path forward depends on resolving this issue. Of course both lower drive and inadequate knowledge of the ablator can be playing a role, as suggested by experimental indications that the drive is overestimated, as discussed below, and the surprisingly poor performance of Ge-doped vs. Si-doped ablators.

If the measured implosion velocity is consistent with the $\mathrm{x}$-ray drive measured at the surface of the capsule, then there is $\sim 200 \mathrm{~kJ}$ of energy unaccounted for, and the energy balance in the hohlraum has to be revisited, as discussed in Section 6.4.2. Here the need for integrating the many physical effects is emphasized, including laser energy deposition, thermal transport (including non-locality) and radiation transport, non-local thermodynamic equilibrium kinetics, magnetic fields, and the effects associated with laser plasma instabilities

\subsection{Status of the Physics}

In indirect-drive inertial fusion, the capsule is imploded by the pressure of $\mathrm{x}$-ray heated ablator material. The $\mathrm{x}$-rays are predominantly absorbed in a narrow region, of order of the Planck x-ray mean free path at the edge of the cold ablator, and the heated material undergoes rapid outward hydrodynamic expansion. The boundary between hot and cold material, the ablation front, eats into the cold material much more slowly than the speed of sound in the x-ray heated material, launching a shock wave ahead of the ablation front and driving the remaining ablator and fuel inward. The capsule shell is essentially an x-ray driven rocket. The scaling of the ablation process is straightforward: balancing the incoming radiation power/unit area $\sim T_{R}{ }^{4}$, where $T_{R}$ is the 
radiation temperature, with the hydrodynamic power/unit area $\sim P c_{s}$, where $P$ is the pressure and $c_{s}$ is the sound speed of the heated mass, gives a scaling $P \sim T_{R}{ }^{4} / \mathrm{c}_{s} \sim T_{R}{ }^{3.5}$. This simple scaling is approximately verified in simulations and experiments, but material properties can cause variations from the simple model. For instance, opacity of the ablator blowoff can reduce the net radiation flux reaching the ablation front by absorbing and re-emitting incident $\mathrm{x}$-rays back into the hohlraum. The internal energy of the heated material represents energy unavailable to do hydrodynamic work, so the EOS of the hot ablator affects ablation pressure. The EOS of the cooler part of the ablator plays a critical role in shock wave propagation ahead of the ablation front.

The NIC point-design ablator [1] is composed of glow-discharge polymer, essentially slightly hydrogen-rich $\mathrm{CH}$ plastic, with embedded dopant layers of either silicon or germanium. The ionization energies of carbon $\mathrm{K}$-shell electrons are many hundreds of $\mathrm{eV}$, contributing significantly to the hot ablator internal energy until the carbon is fully stripped above electron temperatures of $130 \mathrm{eV}$. Errors in ionization state can translate into errors in ablation pressure. As discussed in the sidebar, non-LTE effects [2] in the ablator due to the high radiative flux with $\mathrm{T}_{e}<\mathrm{T}_{R}$ can lead to significant changes in the ionization state. Even LTE opacities and EOS, calculated with detailed quantum models, have significant uncertainty. Opacities of both the carbon and higher-Z dopants, calculated with codes such as OPAL [3] and VISTA [4], can have up to $20 \%$ uncertainties in the Planck or Rosseland means for the range of interest. The EOS models produced by codes such as the spherical ion Purgatorio code [5] or the simpler QEOS Thomas-Fermi-based model [6] have significant uncertainties. Better models of EOS, opacity and non-LTE effects represent scientific opportunities where improved understanding can impact progress toward ignition.

The primary issue is that the experimentally observed implosion velocities are lower than predicted by the baseline 1D simulations. The estimated remaining mass - though higher than predicted - is close to being consistent with the measured implosion velocity, suggesting that the "rocket" model is performing nearly as expected, i.e., for a rocket with exhaust speed, $\mathrm{c}_{s}$, we expect a shell velocity $\sim \mathrm{c}_{s} \ln \left(\mathrm{M}_{0} / \mathrm{M}_{\text {remain }}\right)$, where $\mathrm{M}_{0}$ and $\mathbf{M}_{\text {remain }}$ are the initial and remaining shell mass.

Dante x-ray drive measurements appear to be reproduced by simulations based on the high flux model [7], as discussed earlier. However, there is a general concern that this agreement is due to canceling errors, such as an over predicted LEH closure balancing excess $T_{R}{ }^{4}$, since Dante measures essentially the product of the open LEH area and the radiation power/unit area, $T_{R}{ }^{4}$. Timeintegrated soft $\mathrm{x}$-ray images suggest that the LEH closure is less than that predicted by simulations. If this is the case, it would suggest that the x-ray flux seen by the ablator is not the same as that measured by Dante.

The observed implosion velocities and shock timing can be reproduced by performing simulations that modify the drive flux using temporally dependent flux multipliers, i.e., tuning the drive to match the measured trajectory and shock timing. These flux multipliers adjust the measured flux by more than $50 \%$ in various temporal windows, especially as the capsule approaches peak compression. It seems likely that the flux multipliers are masking some combination of errors in the EOS or opacity, or reflecting an actual drive reduction compared to simulations. To reproduce the observed velocity and shock timing, approximately $200 \mathrm{~kJ}$ of laser energy must be discarded. Where did it go? Even with this reduced drive, the measured neutron yields are $20-50 \%$ of those predicted, leading to a secondary question about whether the reduced neutron yield and associated low stagnation pressure are due to uncertainties in the ablator physics or to 2- or 3-dimensional effects.

\subsection{Opportunities for Progress}

Dedicated experiments, as well as improved models, are required to understand and resolve the "1D" physics issues as defined in the introduction. Issues include:

- The relationship between the Dante measurement and the radiation seen by the capsule is unknown. Since the Dante instrument measures the time-dependent total power, resolved roughly in $\mathrm{x}$-ray energy by employing 
an array of x-ray diodes with different filters, the interpretation of the data involves

knowledge of the LEH area and the difference in the radiation flux shining out the LEH and that incident on the capsule. Both the LEH area and the radiation distribution within the hohlraum are typically obtained from code calculations. There are indications from timeintegrated soft $\mathrm{x}$-ray images that the LEH area, which decreases in time due to ablation of gold around the LEH opening, is being undercalculated. Simulations with the drive lowered consistent with the greater LEH area have smaller drive discrepancies, i.e., the multipliers are closer to unity.

- The distribution of radiation within the hohlraum depends on the location of the reemitting surface, of order optical depth unity into the ablated hohlraum wall. But the wall undergoes significant hydrodynamic motion even with the tamping action of the fill gas and ablated capsule material. If the fill gas and ablated hohlraum wall material mix - an effect not usually resolved in calculations - capsule view factors could change. A related issue is the level of uncertainty in the distribution of laser beam energy and high-temperature emitting material.

- Ablation pressure is controlled by the EOS, opacity and non-LTE kinetics of the ablator material, and predictions have been found to be model-dependent. One ablator opacity model, the Detailed Configuration Accounting (DCA) model [2], gives rise to a double ablation front structure during the foot and second rise of the $\mathrm{x}$-ray drive, i.e., the pressure vs radius has two peaks. This result evidently follows from a strong frequency dependence of the opacity caused by carbon K-shell physics, which leads in turn to separate loci for the absorption of different parts of the incident spectrum. Such a double-peaked structure, if real, could alter the shock history and capsule stability in ways that are difficult to predict. The opacity models interact with radiation transport models since the radiation must pass from the hohlraum wall through an extended atmosphere of flowing ablated material. In addition, the composite structure of the ablator, with multiple layers of different dopant concentration and density, stresses the EOS and opacity models, e.g., by leading to multiple shock reflections at interfaces.

- Preheat of the capsule by electrons and hard xrays is a concern. The ablator must be doped with higher-Z material to optimize preheat protection against gold M-band emission between 2-4 keV. Preheating can both raise the entropy of the fuel, limiting the ability to compress it to the required $\sim 500$ GBar, and alter the ablator dynamics by, e.g., thickening the ablator in flight. This remains an important issue since successful ignition depends on knowledge and control of density profiles of both the fuel and ablator. The amounts and distribution of $\mathrm{x}$-rays $>4 \mathrm{keV}$ and hot electrons are less well known than the softer parts of the $\mathrm{x}$-ray spectrum. Time-integrated hard x-ray measurements show that a significant population of hot electrons exists.

- The shell trajectory, thickness and inferred remaining mass are measured in the convergent ablator (ConA) experiments where a "sym cap" or capsule with the cryogenic fuel replaced by an equivalent mass of plastic is backlit with multi-keV $\mathrm{x}$-rays [8]. The remaining mass inferred from the experiments provides a check against the expected rocket efficiency as discussed above.

Yet there are substantial error bars on the measurement, especially toward the end of the implosion where the shell thickens.

Improvements in remaining mass measurements could more tightly constrain models.

- Shock timing is set in "keyhole" experiments that use a Velocity Interferometer System for Any Reflector (VISAR) to measure shock propagation in liquid deuterium through the 4th shock pulse. However the technique stops working as the 4 th shock propagates through the target due to blanking from preheat and/or high shock temperatures. The pressure history later in time remains unknown, but could play a role in the observed low stagnation pressure by, for example, generating an additional shock that deposits energy in the capsule interior. The extra energy would cause the capsule to "bounce" at lower stagnation pressures.

The most important opportunity is to "break" the problem at the ablation surface by determining 
whether the $\mathrm{x}$-ray drive on the capsule is consistent with Dante measurements or with implosion velocity measurements. If the radiation drive at the capsule surface is consistent with the measured implosion velocities then the highest priority is to understand why the drive is less than predicted, and whether ablator physics plays a role in the deficit in neutron yield. If the drive agrees with Dante measurement, then the priority is to pursue the radiation-ablator coupling physics. Of course, it may not be possible to cleanly separate these two issues, but this "decision-tree" should indicate which issue is most important and the one to tackle first

Table 1 shows the matrix that describes this path.

Table 1: The basic decision matrix for understanding the x-ray transport and ablation physics issues including the lower than predicted implosion velocities. The parentheses indicate the relevant PRD described below.

\begin{tabular}{|l|l|l|}
\hline $\begin{array}{c}\text { (1) Is the measured } x \text {-ray drive } \\
\text { consistent with observed } \\
\text { implosion velocity? }\end{array}$ & Yes & No \\
\hline \multirow{2}{*}{} & $\begin{array}{c}\text { (2) Can ablator physics } \\
\text { contribute to low yields? }\end{array}$ & $\begin{array}{c}\text { (3) What ablator physics is } \\
\text { contributing to reduced coupling? }\end{array}$ \\
\cline { 2 - 3 }
\end{tabular}

\subsection{Priority Research Directions}

\subsubsection{Capsule X-ray Drive}

It is of the highest priority to resolve the question of whether the observed implosion velocities are the result of missing energy of up to $200 \mathrm{~kJ}$ in the $\mathrm{x}$-ray drive, or if energy is failing to couple efficiently to the ablator. At this point, this is primarily an experimental question, though its answer will drive, and in fact set priorities for improving theory and modeling.

The critical step in addressing this question is an experiment to obtain a direct measure of the relation between the $\mathrm{x}$-ray flux at the capsule and the flux observed at the LEH of an ignition hohlraum. An example is the Viewfactor experiment, which can be fielded in the near-term, using existing NIC platforms and diagnostics.

The Viewfactor experiment employs a NICscale hohlraum with one end cut off, exposing the hohlraum interior and far LEH to views from Dante and $\mathrm{x}$-ray imaging diagnostics (see Viewfactor Experiment sidebar). The hohlraum extends $2 \mathrm{~mm}$ past the midplane of the capsule, which is replaced by a thin plastic shell to reproduce the plasma environment of an ignition hohlraum. The hohlraum is at cryogenic temperature with He gas fill as in the NIC design, with thin plastic windows to confine the gas. The hohlraum is driven with a standard ignition pulse shape. From the LEH side, the drive includes the usual complement of 96 beams, while the open side includes only the 23 and 30 degree beams, a total of 32 that strike the hohlraum interior near the capsule equator. Simulations show that density and temperature profiles within the closed end of the Viewfactor target are very similar to an ignition target with about a $20-\mathrm{eV}$ drop in radiation temperature at peak drive due to the larger open area.

The target is diagnosed with the Dante x-ray drive diagnostics that view the hohlraum from the 64 degree (top) and 37 degree (bottom) angles. By conducting two experiments rotated 180 degrees from each other, the Viewfactor platform will provide views at two different angles of both the hohlraum interior near the capsule and the LEH, allowing benchmarking of models that relate the LEH flux to the capsule flux.

The open geometry allows for effective imaging of time-dependent LEH closure viewed both internally and externally. Information from this measurement will shed light on the fidelity of simulations, and, in particular, help determine whether the current agreement between predicted and measured drive is fortuitous. Most information on the LEH closure at his point is from time integrated $\mathrm{x}$-ray images, which is insufficient to pin down time-dependent flux 
multipliers used in the capsule modeling. Limited data from time-gated images exists and more should be collected on capsule or keyhole experiments when possible to help determine time-dependent closure. The Viewfactor experiments will be imaged with the GXD (Gated X-ray Diagnostic) in the Polar DIM (Diagnostic Insertion Manipulator). The open geometry should allow a clear view of the LEH as seen from the hohlraum interior as well as the usual exterior view. The experiment also eliminates the ambiguities caused by looking through two LEHs.
Another valuable experiment would be a direct spectral measurement through a keyhole, a reentrant cone placed in the hohlraum wall that would provide a view of the opposing interior surface of the hohlraum. The experiments could be done with and without a capsule mounted on the keyhole. The design issue for these experiments is closure of the diagnostic line of sight due to x-ray ablation of the cone tip. Direct spectral measurements through the LEH on capsule or keyhole experiments would offer additional information and be easier to field.

\section{Viewfactor Experiment}

The Viewfactor experiment employs a NIC-scale hohlraum with one end cut off, exposing the hohlraum interior and far LEH to views from Dante and x-ray imaging diagnostics. The hohlraum extends 2 mm past the midplane of the capsule, which is replaced by a thin plastic shell to reproduce the plasma environment of an ignition hohlraum. The hohlraum is at cryogenic temperature with He gas fill as in the NIC design, with thin plastic windows to confine the gas. The hohlraum is driven with a standard ignition pulse shape. From the LEH side, the drive includes the usual complement of 96 beams, while the open side includes only the 23 and 30 degree beams, a total of 32 that strike the hohlraum interior near the capsule equator. Simulations show that density and temperature profiles within the closed end of the Viewfactor target are very similar to an ignition target with about a $20 \mathrm{eV}$ drop in radiation temperature at peak drive due to the larger open area.

The target is diagnosed with the Dante x-ray drive diagnostics that view the hohlraum from the 64 degree (top) and 37 degree (bottom) angles. By conducting two experiments, rotated 180 degrees from each other, the Viewfactor platform will provide views at two different angles of both the hohlraum interior near the capsule and the LEH, allowing benchmarking of models that relate the LEH flux to the capsule flux. The open geometry also allows for effective imaging of time-dependent LEH closure viewed both internally and externally. 
It might also be possible to constrain models of the x-ray drive though a series of measurements at different locations along the hohlraum wall using mounted planar targets viewed with a VISAR, or with a shock breakout diagnostic or streaked optical pyrometer.

Once experiments have determined the $\mathrm{x}$-ray drive at the capsule, the result determines the subsequent path. There are two possibilities:

- If the velocity measurements are consistent with the $x$-ray flux incident on the capsule, then the focus should be on understanding why the incident flux is less than predicted and inconsistent with the Dante measurements. An important question is: where did the "missing" $200 \mathrm{~kJ}$ go? Research directions addressing this question are detailed in the report of Panel 6, as PRD 2, Section 6.4.2. It also becomes a priority to investigate features of the ablator response that might effect neutron yield, as discussed in the next section.

- If the measurements show that the x-ray flux on the capsule is, in fact, consistent with the Dante measurements, then there is likely a problem with out understanding of the conversion of radiation to pressure at the ablation surface. For example, the detailed modeling of the atomic physics of carbon is difficult in this regime owing to the complex interaction between the radiation field and level populations. Ablator radiative coupling is discussed at PRD 3, below in Section 6.4.3.

\subsubsection{Ablator Hydrodynamics}

If the measured radiation drive at the capsule substantially explains the velocity, then the highest priority becomes understanding why the yield is still low, and whether ablation phenomena are playing a role. Relevant questions include whether there are ablator physics issues that could affect the 1D performance, whether the 1D profiles are sufficiently different than predicted in nominal simulations that strong higherdimensional effects like Rayleigh-Taylor (RT) instability and mix are triggered, or whether small 3D effects exist that are not included in models but could be amplified by instability growth or spatial convergence.
Ablator issues that could affect the yield include:

- Additional shocks. The shock history is not measured at late times and, as noted above, one or more additional shocks could raise the entropy and early-time energy of the hotspot enough to prevent effective compression.

- Plasma density scale and ablation rates determine RT growth near the ablation front and may differ from models.

- Unstable density profiles. The Atwood number, related to the density jump at the ablator/ice interface or other interfaces, may be less favorable than predicted in nominal models, leading to enhanced RT growth and mix.

- 3D seeding of instabilities. Microstructure of the ablator may lead to perturbations in the flow once the shocks pass through the material, providing a larger seed for instability growth than the surface finish alone.

- 3D seeding of asymmetry and non-radial flow. The concern here is that drive asymmetries during the foot of the pulse and at intermediate mode numbers, i.e., due to beam imbalances, are difficult to measure and characterize. The variations in flux could cause significant angular variation in fuel adiabat, which is a sensitive function of the laser-foot intensity, as well as introduce small tilts in the capsule surface normal. A tilt of 2 degrees early in time is enough for a portion of the shell to effectively "miss" the geometric center once the main drive is applied. Energy accounting of the capsule at stagnation suggests that a significant fraction of the implosion energy is not effectively converted to internal energy (proportional to pressure times volume or $P V$ ), possibly due to misdirected mass, or equivalently, non-radial flow.

Experiments addressing these issues could include a variant of the Crystal ball, employing the doped ablator and improved resolution ConA experiments. Characterizing 3D effects due to microstructure or early-time asymmetry might be possible using variants of the keyhole and Crystal ball platforms that extend the angular view of VISAR that probes the interior 


\section{Crystal Ball Experiment}

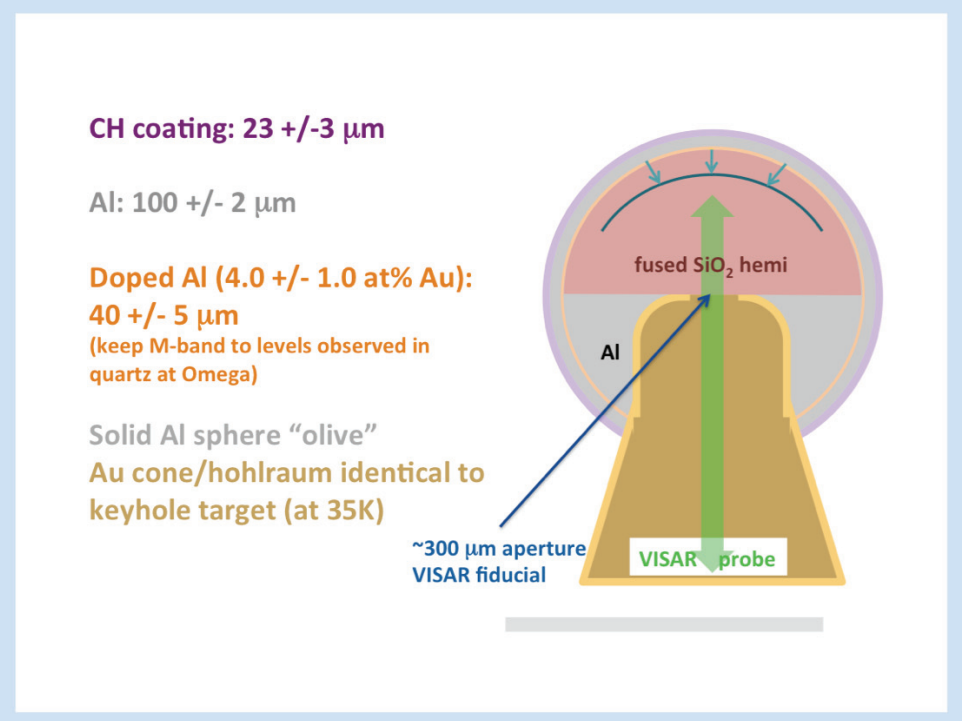

The Crystal ball experiment builds off the successful NIC keyhole geometry, inserted into a NIC hohlraum, used to study shock history in the ablator and fuel via VISAR viewing the interior of the target. In the Crystal ball, the liquid deuterium of the keyhole target is replaced with a fused silica hemisphere, which is expected to allow probing to higher shock pressures, perhaps through the peak of the drive, before blanking of the VISAR occurs due to M-band preheat or shock heating of the material. Two versions of the target are being pursued, both driven by a standard ignition pulse. The first uses an aluminum ablator, with a thin plastic overcoat to reproduce the NIC plasma environment. The aluminum serves as a "standard" for drive characterization and has been used successfully in the past on other NIF experiments. The second version uses the standard NIC glow-dischargedoped polymer ablator to allow characterization of the pressure drive at the ablator interior. The second version will help isolate the effect of the choice of the specific ablator material, which has uncertainties in EOS and opacity.

surface of the capsule. VISAR provides a very precise velocity time history that could reveal subtle 3D effects if there is sufficient angular coverage and resolution. Developing improved models of ablator EOS, opacity and non-LTE effects would also constitute an important component of this research direction.

\subsubsection{Ablator Radiative Coupling}

If the measured radiation does not explain the low implosion velocity, the problem is likely to be the coupling of the radiation drive to the ablator. It would be useful, in this case, to directly measure the response of the capsule to the drive, including ablation rate measurements with "halfraums," as was done on OMEGA [9], but using ignition-like drives at NIF. Opacity uncertainties in the ablator could be reduced by time-dependent spectroscopy of the capsule reemission, as well as dedicated
OMEGA experiments to measure spectrally dependent ablator emission and absorption opacities.

A possible deficiency in our ability to predict radiation coupling is the treatment of non-LTE effects. For example, in the NIC point design, the ionization balance of the carbon in the ablator is difficult to calculate due to the non-LTE nature of its atomic kinetics. Experiment using the Crystal Ball, ConA, and shock-timing keyhole platforms, should be carried out with Be or other low-Z materials to normalize these effects in systems that are "easier" to ionize. Better non-LTE kinetics models would be helpful in understanding the results of these experiments.

The composite structure of the doped ablator package also presents modeling challenges. The propagation of shocks in multilayered samples 
stresses the understanding the EOS, requiring accurate knowledge of release states as well as the Hugoniot. Planar experiments with composite layers could help sort this out. Furthermore, the opacity and EOS models currently used in simulations of the ablation process are not consistent with each other. For example, they generally use different average ionization states. This could be improved in the plasma regime, for instance, by using the EOS implicitly generated by an opacity code in the course of calculating the energy levels and populations. Release physics of the DT as well could be important in determining how energy is coupled to the hotspot.
Preheat by hot electrons or deep penetration by a non-Planckian radiation drive (e.g., M- or Lshell lines from hohlraum) could raise the fuel adiabat, thicken the ablator in-flight and induce asymmetries. Early indications from the Crystal Ball experiment suggest that substantial hard $\mathrm{x}$ ray or hot electron preheat is penetrating the capsule. Time and spectrally dependent hard xray measurements are needed to help quantify hot electron production. Other experiments in keyhole geometry can detect preheat that penetrates the capsule using "calibrated" materials interior to the capsule that lose transparency to laser light at known volumetric energy absorption.

\section{Non-LTE Effects}

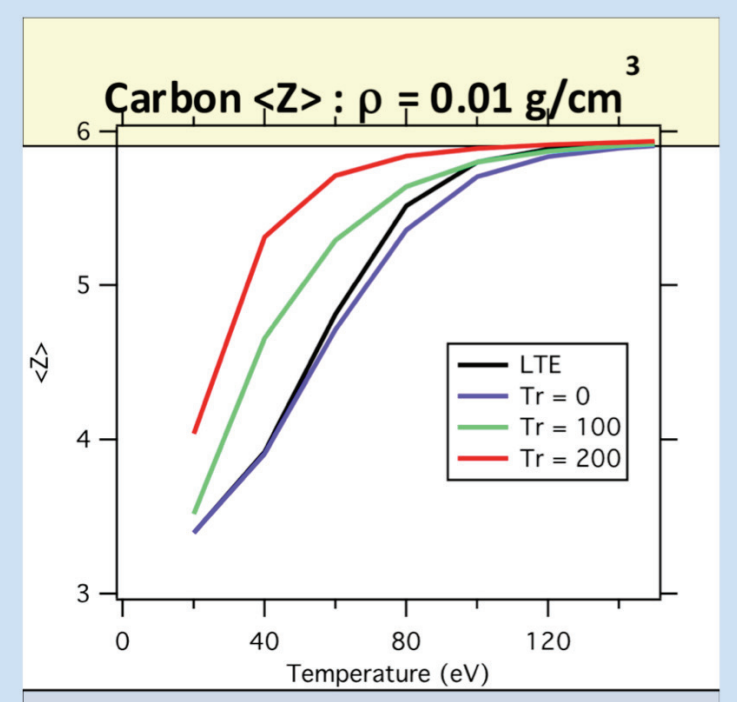

\section{Radiation drives the material away from LTE}

Non-local thermodynamic equilibrium (non-LTE) effects manifest themselves through changes in the atomic kinetics of the plasma. When collisions with thermal electrons are the dominant atomic processes responsible for atomic excitation and ionization (and de-excitation and recombination), the plasma is in LTE. Thus, as the density increases, plasmas approach LTE. Radiative processes driven by atomic processes in the plasma or by an intense radiation field with radiation temperature larger than the plasma temperature move the plasma away from LTE. In general, non-LTE effects become important for low densities, high radiation fields and/or large transition energies. In NIF plastic ablator plasmas, the differences between LTE and non-LTE in the absence of the radiation field are modest. However, when a radiation field with a temperature characteristic of a NIF hohlraum is included in the model calculation, the atomic kinetics of the carbon in the plastic ablator become non-LTE and the ionization increases substantially relative to the case without radiation field. Non-LTE effects impact opacity, which affects radiation transport, equation of state and thermal transport. Lack of consistency between the microscopic, atomic physics description of these phenomena is an issue that requires attention since it impacts implosion hydrodynamics. The figure for the average ionization state of carbon was produced by $\mathrm{H}$. Scott from LLNL using the Cretin non-LTE atomic kinetics code. In the figure, $T_{r}$ is the radiation temperature. 
Finally, thermal transport in the ablator also needs to be explored since some portions of the ablator expand into parts of the hohlraum directly heated by the laser, where the high electron temperature is high, and electron thermal conduction dominates over radiative transfer.

\subsection{Conclusions}

The highest priority for understanding why the implosion velocity is lower than predicted is to measure the $x$-ray radiation incident on the capsule. That will determine a path forward for understanding what accounts for the "missing energy." The path toward understanding the $\mathrm{x}$-ray drive as well as a better understanding of how the ablator behaves has been outlined above in the areas of capsule $\mathrm{x}$-ray drive, ablator hydrodynamics, hohlraum energetics and ablator radiative coupling. A good understanding of what is referred to as " $1 \mathrm{D}$ " physics of the ablator and fuel forms the underpinning for understanding ICF capsule ignition physics as a whole. In fact, it would be desirable to consider a simpler implosion system - perhaps employing ramp compression, or a single shock - to try to establish an easily understandable baseline from which to test our basic understanding. Subtle 3D effects such as ablator microstructure or early time drive asymmetry may play a role in seeding deviations from "1D." The multi-dimensional physics of asymmetry, instability and mix that is thought to ultimately limit capsule performance cannot be well modeled or characterized without knowledge of both the "unperturbed" state and initial or early time conditions of the matter before these processes become dominant.

\subsection{References}

1. S. W. Haan, J. D. Lindl, D. A. Callahan, D. S. Clark, J. D. Salmonson, B. A. Hammel, L. J. Atherton, R. C. Cook, M. J. Edwards, S. Glenzer, A. V. Hamza, S. P. Hatchett, M. C. Herrmann, D. E. Hinkel, D. D. Ho, H. Huang, O. S. Jones, J. Kline, G. Kyrala, O. L. Landen, B. J. MacGowan, M. M. Marinak, D. D. Meyerhofer, J. L. Milovich, K. A. Moreno, E. I. Moses, D. H. Munro, A. Nikroo, R. E. Olson, K. Peterson, S. M. Pollaine, J. E. Ralph, H. F. Robey, B. K. Spears, P. T. Springer, L. J. Suter, C. A. Thomas, R. P.
Town, R. Vesey, S. V. Weber, H. L. Wilkens, and D. C Wilson, "Point Design Targets,

Specifications, and Requirements for the 2010 Ignition Campaign on the National Ignition Facility," Phys. Plasmas 18, 051001 (2011).

2. H. Scott and S. Hansen, High Energy Density Phys. 6, 39 (2010).

3. C. A. Iglesias and F. J. Rogers, Astrophys. J. 464, 943 (1996).

4. VISTA is a relativistic LTE opacity code developed by M. H. Chen based on STA theory [A. Bar-Shalom et al., Phys. Rev. A 40, 3183(1989)] using Dirac-Hartree-Slater methods with Breit and QED corrections for the atomic data.

5. B. Wilson, V. Sonnad, P. Sterne, W. Isaacs, Journal of Quantitative Spectroscopy and Radiative Transfer, Volume 99, Issue 1-3, p. 658-679.

6. R. More, K. Warren, D. Young, and G. Zimmerman, Phys. Fluids 31, 3059 (1988).

7. M. D. Rosen, LLNL Technical Report No. LLNL-PRES-428527 3/18/10, 2010. R. P. J. Town, M. D. Rosen, P. A. Michel, L. Divol, J. D. Moody, G. A. Kyrala, M. B. Schneider, J. L. Kline, C. A. Thomas, et al. Phys. Plasmas 18, 056302 (2011).

8. D. G. Hicks, B. K. Spears, D. G. Braun, R. E. Olson, C. M. Sorce, P. M. Celliers, G. W. Collins, and O. L. Landen,"Convergent Ablator Performance Measurements," Phys. Plasmas 17, 102703 (2010)

9. R.E.Olson, G.A. Rochau, O.L. Landen, and R.J. Leeper Phys. Plasmas 18, 032706 (2011). 


\subsection{PANEL 3 - IMPLOSION HYDRODYNAMICS}

\subsection{Introduction}

Using an ablation pressure of $\sim 100$ Mbars over a period of $\sim 10 \mathrm{~ns}$, the implosion hydrodynamics of an ICF capsule have always been a balance between obtaining high implosion velocities $(>370 \mathrm{~km} / \mathrm{s})$ necessary to create sufficient fuel densification $\left(\rho R>1 \mathrm{~g} / \mathrm{cm}^{2}\right)$ and stagnation pressure ( $>400$ Gbars), while simultaneously attempting to avoid hydrodynamic instabilities and avoid raising the DT fuel entropy much -- either of which frustrates fuel densification and hotspot creation. Ideally, an ICF implosion should have yield within about $50 \%$ of $1 \mathrm{D}$, but present evidence from NIC suggests that is not the case, and it is presently difficult to estimate the degree to which irradiation asymmetries or high-mode mix, respectively, are contributing to this behavior. If the NIC implosion can resolve any low-mode implosion hydrodynamics issues, there remains the significant challenge of achieving the needed convergence ratios in excess of $\geq 30$ without generating unacceptable mid- to high-mode instability growth. Indeed, for those NIC shots in which the implosion speed was pushed up above $\sim 300 \mathrm{~km} / \mathrm{s}$ and had less than $8 \%$ ablator mass remaining, hotspot ion temperature and yield both dropped significantly, with an increase in x-ray brightness, which is consistent with excessive perturbation growth due to hydrodynamic instabilities.

\subsection{Status of the Physics}

Presently, simulations can only approximately predict the ablation pressure history during the laser pulse even in a 1D average sense. Since code inadequacies were known before the NIC campaign began, several experimental platforms were developed to normalize the implosion as necessary. Data needed to normalize the implosion come from convergent ablator (ConA) trajectory experiments that measure ablator radius, $R(t)$, implosion velocity, $V_{\text {imp }}(t)$, and thickness, as well as experiments that measure velocity of a leading shock up to the time of coalescence of the fourth shock launched during the rise of the main drive to peak power. It appears that the ablation pressure of the implosion is currently over predicted by $\sim 1.5 \mathrm{X}$ during the $2^{\text {nd }}$ pulse and the $4^{\text {th }}$ rise acts as if delayed and reduced, with the peak radiation drive being over-predicted by $\sim$ $15 \%$ in flux. This could be due to some combination of hohlraum physics affecting the $\mathrm{x}$ ray drive incident on the capsule and/or the efficiency of the ablator of converting x-ray energy into ablation pressure through ionization. In addition to influencing the apparent efficiency of the implosion, the detailed temporal history of the ablation pressure or ablator response can affect the fuel adiabat $(\rho R)$ and mix.

A performance cliff attributed to mix has been observed at an implosion velocity of $\sim 300 \mathrm{~km} / \mathrm{s}$ in several NIC implosions. Simulations in 2D have not been able to assess correctly the observed mix. Experimentally, the observed mix could be due to a combination of factors including less efficient rocket (more mass ablated at given velocity, hence more feed-through), more ablation front growth possibly due to unfavorable 1D pulse shaping, 3D effects that are not included in current 2D simulations, and enhanced growth in thin spots due to low mode asymmetry. Theoretically, it is possible that the simulation predictions of instability growth are too optimistic possibly due to (but not limited to) overestimating the smoothing effects of ablation-front stabilization or insufficiently representing the structure on and in the ablator and ice (coming from manufacture or from physics such as species separation effects).

Evidence from nuclear spectra from multiple lines of sight suggests significant $\rho R$ asymmetry in the assembled cold fuel (with the $\rho R$ at the pole of the capsule measured to be $\sim 2 \mathrm{x}$ that of the waist). This can reduce the efficiency with which implosion kinetic energy is converted to stagnation pressure, resulting in lower stagnation pressures, densities, and yields. For the inferred in-flight fuel adiabat $\sim 1.5 \pm 0.1$, the inferred hotspot density is $\sim 2 \mathrm{x}$ too low, and the fuel $\rho R$ is low by $\sim 20 \%$. A number of factors could contribute to this including (but not limited to), mix, low mode asymmetry (hotspot volume) and 
associated vortex flows

$\left(p_{3 D} \sim p_{1 D}-\frac{1}{2} \rho v_{\text {non-radial }}^{2}\right)$, kinetic effects as well as a significant " 5 th shock".

\subsection{Opportunities for Progress}

Uncertainties in laser propagation and $\mathrm{x}$-ray conversion physics (Panel 1) and $\mathrm{x}$-ray transport and ablation physics (Panel 2) compound uncertainties in implosion hydrodynamics (topic of this Panel) primarily through uncertainty in the drive pressure history as a function of solid angle and time, $P(\theta, \phi, t)$, and preheating of the capsule. With a drive that is calibrated to mimic the capsule trajectory and shock timing observables, it is found that 2D simulations, with measured surface and ice roughness, still over-predict stagnation pressure (but not $T_{i o n}$ ) by $\sim 2 \mathrm{x}$ and yield by $\sim 4 \mathrm{x}$ (with alpha-particle deposition turned off) to $\sim 10 \mathrm{x}$ (with alpha-particle deposition turned on). By construction, these 2D simulations match the measured down-scatter ratio $(D S R)$, which is proportional to $\rho R$.

If drive-related asymmetries can be resolved and the desired time-dependent $1 \mathrm{D}$ ablation pressure is recovered, the problems of the implosion hydrodynamics reduce to managing instability and mix, while delivering the required hotspot formation (via ablation of the inner $\sim 10 \%$ of the DT ice and set by thermal conductivity in implosion kinetic energy) and hotspot stagnation pressure, which is most strongly affected by implosion speed $\left(p_{\text {stag }} \sim p_{a b l}^{1 / 3} v_{\text {imp }}^{3} / \alpha\right)$.

\subsection{Priority Research Directions}

\subsubsection{Investigation and Control of Ablation Front Instability}

It is likely that the ablation front instability is presently under-predicted in simulations for a variety of reasons. Current code simulations indicate that many of the targets now being shot have more simulated ablation front growth than was expected for the NIC point design. More than expected ablation front growth results primarily from higher ablator opacity, which has been increased as a consequence of the higher than expected $\mathrm{x}$-ray preheat. Also, there are strong indications from NIC data that the ablative instability is more unstable than originally expected. Due to the way the ignition capsule hotspot is formed, ablator material only needs to penetrate into the last $10 \%$ of the DT ice to show up in the hotspot.

The following research directions on this topic are recommended:

- Face-on radiography Richtmyer-Meshkov (RM) and Rayleigh-Taylor (RT) experiments with a perturbed ablator and the full NIC drive pulse to compare against simulation predictions. While many high energy density physics experiments have been performed previously for one or two shock RM instabilities at an interface, four shock (e.g., NIC-like pulse) studies of RM instability have not been performed. Testing the veracity of simulation predictions of the ablator after the passage of the fourth shock is key for correctly calculating the RT instability growth that subsequently follows. Validating simulation unstable growth predictions under multiple shocking is particularly prudent for the NIC capsule point design that presently has many interfaces within the ablator (the dopant layers). Since ICF target design trade-offs of doping levels, doping profile, and shell thickness are made based upon simulation predictions of unstable growth, it is clearly desirable for the predictions to be as correct as possible.

- Design and shoot a series of implosions with varying picket peak to trough ratios to possibly find an implosion less sensitive to ablative Rayleigh-Taylor instability and improved compression. Unvalidated simulations of ICF capsule instability growth presently indicate that the ablation front instability growth factor at peak implosion velocity as a function of mode number can be significantly modified through control of the amplitude of the first picket in the drive pulse with negligible affects on adiabat. Similarly, simulations of ICF implosions with different drive troughs immediately after the 1 st picket show a reduction in growth factor vs. mode number at peak velocity (see Figure 3.1). These results suggest the existence of a potential knob to improve the instability performance of ICF capsules. 

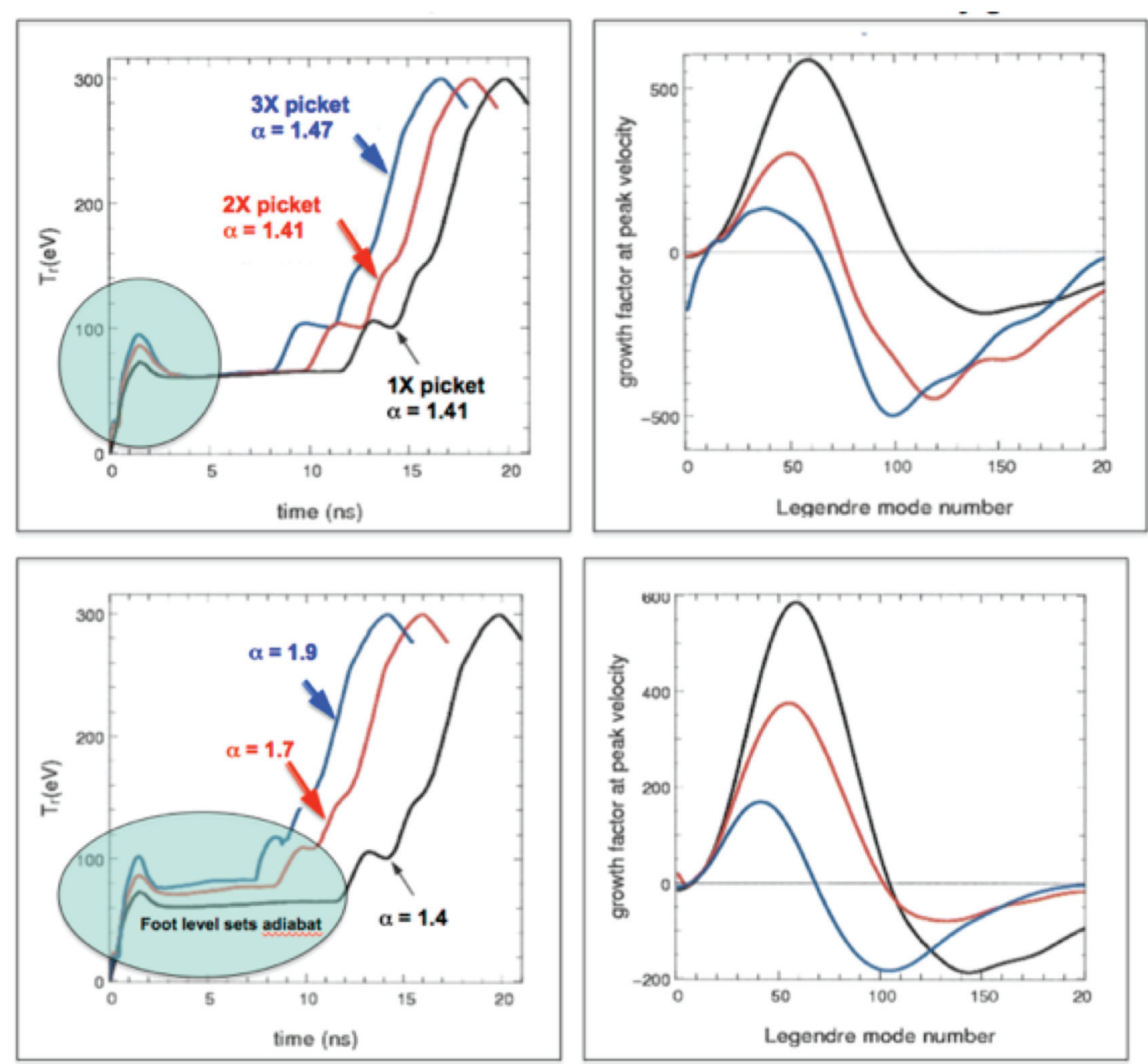

Figure 3.1: Altering the first picket in the drive (upper left) potentially shifts the extrema of the ablation from instability growth (upper right) without much impact on fuel adiabat. Increasing and shortening the foot level of the "trough" of the drive pulse (lower left) potentially reduces the instability growth for many mode numbers (lower right). Figures courtesy of the NIC target design team.

- Thicken the DT ice layer ( $20 \mu \mathrm{m})$ or thicken the ablator and ice together in order to move the hotspot forming inner ice region away from the ablator and delaying the time at which the central shock reflection re-enters the ablator. Thickening the ablator may help to control penetration of material into the hotspot, but owing to the density difference between the DT ice and the ablator, thickening the ice has much less impact on implosion speed than does thickening the ablator. Altering the ice thickness requires no capsule fabrication changes and is a simple test of the depth of penetration of fingers of ablated material.
- Perform planar ablator experiments with graded $\mathrm{CH}$ and alternate ablator materials and diagnose the ablation profile side-on and use VISAR on the face of the ablator to diagnose $v(t)$. Some of the unexpected ablation front instability growth in the NIC point design could be due to poor understanding of the material properties (e.g., equation of state, kinetic effects, etc.) under ICF conditions. Testing alternate ablator materials (in a planar geometry for simplicity and efficiency of target fabrication) would illuminate whether other 
choices of ablator materials behave in a more predictable fashion.

- Test implosions with alternate ablator materials. Presently, beryllium and high-density carbon (HDC) ICF targets are "on-the-shelf," and while they are not necessarily optimized ignition designs, testing them could quickly illuminate questions about hohlraum-ablator coupling and instability growth. In particular, is the observed behavior more-or-less what is expected from simulations? Some target fabrication research and development is required to field other alternate ablator capsules.

- Use scaled implosions (with less energy) to perform a more comprehensive study. Data is at a premium here and since laser glass damage effects are cumulative, more shots can be performed if targets can be designed to use less energy per shot.

\subsubsection{Mix in Extreme High-Acceleration Implosions Driven by Multiple Strong Shocks}

Experience with the simulations of implosions with large Atwood numbers and low remaining mass is that the simulations can produce results that look like "turbulence." For well-designed implosions, simulations do not look turbulent even in 3D with mode numbers up to $\sim 1000$, about where ablative stabilization sets the minimum scale length. When simulations are adjusted to recover the measured 1D implosion hydrodynamics, the results do not look turbulent, but these same simulations still over-predict the measured neutron yields. While turbulence and mix are often associated, diffusion processes and successive folding flows, for example, can produce "mix" in the absence of turbulence. Furthermore, recent theoretical analysis shows that the statistical properties of accelerated mix should depart substantially from canonical turbulence. In particular, when compared to canonical turbulence, accelerated mix has a higher level of correlation, smaller fluctuation contributions, stronger dependence on initial conditions, and steeper spectra.

NIC data show indications of mix at smaller implosion speed than was anticipated by pre-NIC simulations. Clearly, the simulations are missing something. So far, it is not known if the observed mix is "independent" from initial conditions. Obviously capsules with different surface-finish but the same 1D implosion parameters would be the way to determine if mix is independent of initial conditions. On the calculation side, if 2D simulations with an enhanced thermal conduction (to mimic gas/ice atomic mix) successfully explain the data at hand (yield and $T_{i o n}$ ), then it is a strong argument for introducing a sub-grid scale model in the code. In fact, 2D simulations performed with DT thermal conduction multipliers show that a conductivity multiplier of 2.0 gets the $T_{i o n}$ about right, but is still far from getting the right yield [1]. A thermal conductivity multiplier of $\sim 10 \mathrm{x}$ appears necessary to explain the observed yield degradation, but this would drive the $T_{i o n}$ too low. It is possible that excess numerical ablation at the ice-gas interface is a cause of underestimation of hotspot mix.

The panel recommends the following research directions on this topic:

- Perform implosions with increased adiabat of the main fuel to reduce the sensitivity of target performance on mix and obtain a scaling relationship for measured yield/simulated yield (YOC - "yield over clean"). Presently, the NIC point design has focused upon keeping the fuel adiabat as low as possible to obtain the maximum amount of convergence. Stiffer fuel implosions would converge less and likely suffer less from mix. Mapping out the scaling of Y.O.C. with adiabat may provide data useful for mapping out the cliffs associated with mix for the purpose of avoiding such cliffs in subsequent target design iterations.

- Test implosions with roughened ablators and ice and observe the sensitivity to initial conditions. A systematic study in which key target interfaces are roughened would quickly help isolate from where the material responsible for the observed mix cliff originates, helping target amelioration efforts.

- Design and perform implosion experiments with separated reactants (e.g., CD ablator + pure $T$ fill) with a measurement of reaction history if possible. If diagnostically measureable, a separated reactant experiment could quantify the 
amount of ablator material that mixes into the hotspot of the implosion as a function of capsule and drive shape design.

- Develop a large eddy simulation (LES) representation with a parameter free sub-grid model, elaborating the analytical and numerical modeling needed to couple microscopic and macroscopic scales for instabilities and mix induced by strong shocks and strong accelerations.

\subsubsection{Hotspot Formation and Fuel-Shape Physics}

The final phase of the capsule implosion hydrodynamics culminates in the ablation of the innermost part of the DT fuel that creates the hotspot. The optimization that leads to successful hotspot formation is a balance between having enough implosion velocity and a tolerable amount of mix ( 10s of ng). As the imploding shell of fuel decelerates, distortions in the hotspot/main fuel boundary shape grow. Reduced thermal conduction and mass ablation from the shell of fuel into the hotspot could lead to enhanced RT growth during deceleration and smaller stagnation pressures.

Obtaining more data on the hotspot and cold fuel condition are key to resolving issues with NIC capsule performance and model-data inconsistences (see Figure 3.2).

The panel recommends the following research directions on this topic:

- Design and, if justified, test implosions using a small-pore-size doped, wetted foam to enhance imaging of the hotspot and possibly improve the imaging of the cold fuel. Use spectroscopy to obtain direct measurement, through the dopant, of the hotspot density. Presently, hotspot shape in the NIC implosion is only roughly inferred from emission measurements, while the cold fuel shape is not directly known. For the purpose of performing the required low-mode symmetry tuning and for code validation, obtaining accurate imaging of the hotspot and cold fuel shape are highly desirable.
- Perform high-mode "direct" 3D simulation, with known initial conditions, of a practical number of NIC shots and also compare results from various codes using a simplified, but representative, $3 D$ high-mode implosion test problem. Since the observed stagnation pressures is only a factor of 2 above simulation, it is in the realm of possibility that fully $3 \mathrm{D}$ simulations with fully represented initial conditions may, without the need for additional physics, capture the observed degradation of pressure and yield. In particular, a 3D simulation that has no symmetry boundary or axis may have enough non-radial motion (at mesoscopic scales) at stagnation time to explain the stagnation pressure problem.

- Improve the physical database conductivity tables and address more quantitatively the effects of magnetic field on electron conduction. Inaccurately representing electron thermal conductivity can obviously impact the simulations ability to calculate the transfer of heat in an implosion and impact the formation of the hotspot plasma that originates from the inside layer of the DT ice.

\subsection{Conclusions}

To demonstrate gradually increasing performance of ICF targets on NIF will require improvements in many aspects of the implosion hydrodynamics that can be carried into the next stage of hotspot formation and stagnation. The exact steps needed to be taken will depend upon what is found from the experiments and studies outlined above. The elements described above hold the highest leverage on improving the implosion hydrodynamics of ICF targets on NIF.

\subsection{References}

1. D. Clark, Private Communication 2012

2. C.A. Meakin and D. Arnett, Ap. J., 667 (2007) 448 

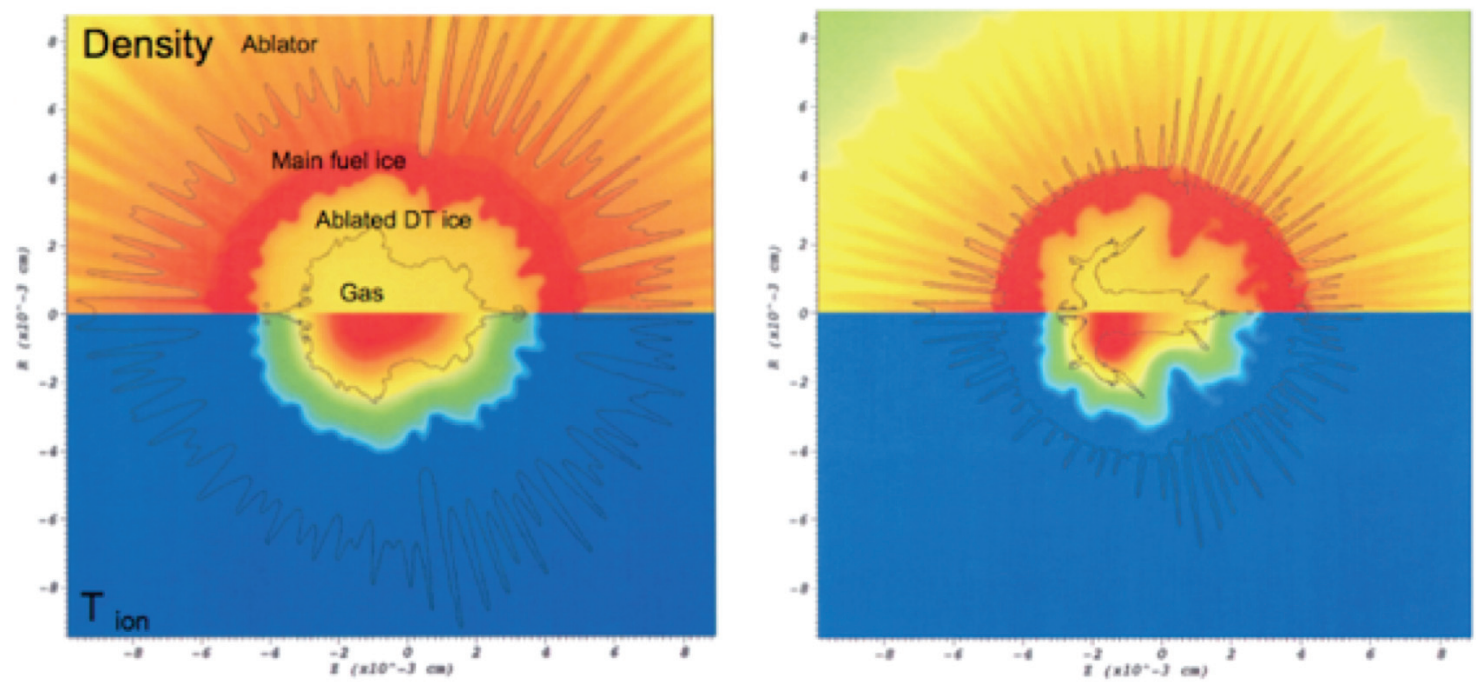

Figure 3.2: A 2D mode 100 ARES simulation of an ICF implosion with realistic surface roughness shows the expected late-time implosion morphology and hotspot. Prior to self-heating from alpha-particle deposition, mechanical pdV work produces heating that spreads radially outward into the DT fuel via electron conduction (assuming conduction and radiation losses don't dominate). This ablated fuel forms the hotspot and is many times the mass of the DT gas that occupies the volume at the center of the capsule.

\section{The Difference between 2D and 3D Calculations and Physics}

Simulation of ICF implosions need integrated modeling using complex multi-physics codes. The physics operates over scales from the hohlraum size to the hot-spot radius. With present capability of computing, to achieve useful time-to-solution of the modeling, assumptions are generally made that the physics can be approximated with azimuthal (along the hohlraum axis) symmetry in two-dimensions (2D).

However, the physics of hydrodynamic motion are very different in $2 \mathrm{D}$ and 3D. An example is shown from astrophysics where there are significant differences in flow morphology and velocity amplitude in simulations of stellar burning, although the size of the mixed region was similar in both 2D and 3D. An active area of future research will be to appropriately link more realistic 3D hydrodynamic simulations to the integrated modeling and better calculate effects of instability and mix.

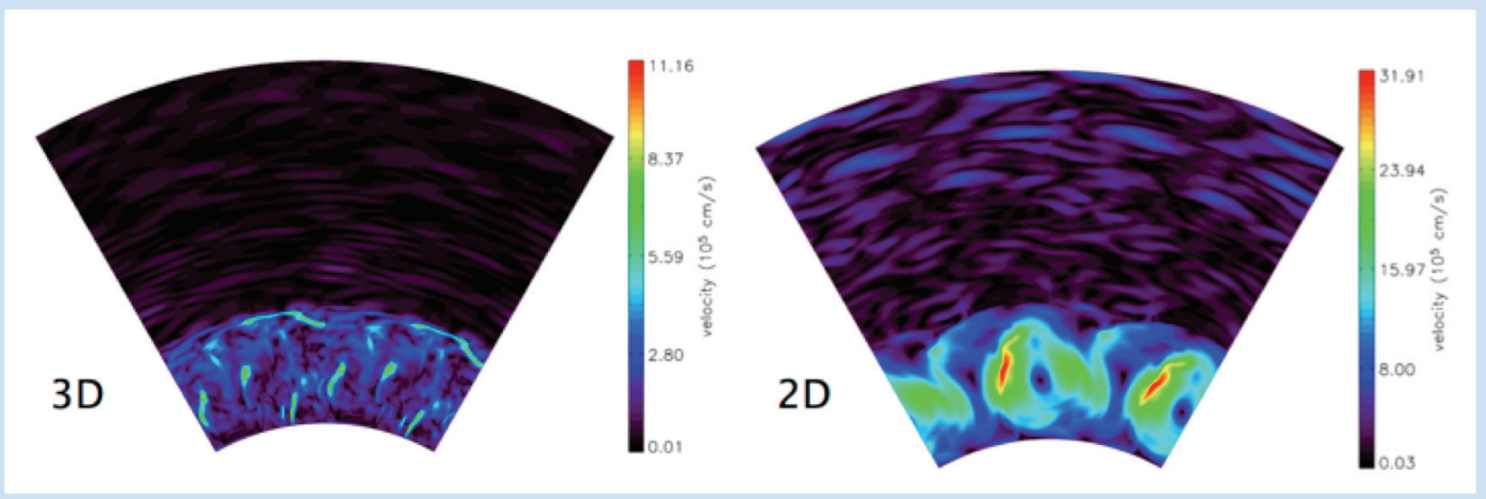

Figure from C.A. Meakin and D. Arnett, Ap. J., 667 (2007) 448 [2]. 


\subsection{PANEL 4 - STAGNATION PROPERTIES AND BURN}

\subsection{Introduction}

To achieve hotspot ignition of an inertial confinement fusion (ICF) implosion, it is necessary to maintain the deuterium-tritium (DT) fuel on a low adiabat during the implosion and to ensure that the shell kinetic energy is efficiently converted to hotspot thermal energy upon stagnation. Analysis of the cryogenically layered capsule implosions performed to date on NIF indicate, first and foremost, that the stagnation pressure of the hotspot and cold fuel are significantly lower than predicted, even when the models are tuned to reproduce shock timing and implosion velocity measurements. An understanding of the physical basis of this pressure deficit is essential to achieving ignition.

\subsection{Status of the Physics}

\subsubsection{Underlying processes and properties}

Insight into the underlying physics of fuel assembly can be gained by studying a zerodimensional (0D) physics model, in which an incompressible, infinitely thin fuel shell compresses a small mass of DT gas at a given initial pressure. In this model, it can be shown that stagnation pressure is inversely related only to the density of the DT gas present upon stagnation. The DT gas pressure is in turn controlled by the initial configuration of the capsule, the DT equation-of-state, the 1D pressure profile of the shell as determined by shock timing and capsule dynamics, and by 3D effects, discussed further below.

Shock mistiming or the presence of extra shocks can affect the stagnation pressure by introducing additional DT mass from the solid fuel layer early in the implosion. Shock timing is achieved empirically using a surrogate liquid D2 target that may give rise to surrogacy errors. In particular, this technique does not account for the shock release at the DT ice surface. Correct shock timing also relies on correct modeling of the shock propagation and density profile of the $\mathrm{CH}$ ablator and DT fuel to set the experimental requirements. There is a possibility that kinetic effects, especially during the final strong shock leading to formation of the hotspot, can modify shock propagation compared to hydrocode predictions. The density profile largely depends on the accuracy of the equation of state for both DT and the ablator materials and may be further impacted by uncertainty in the ablator dopant opacity and preheat. In addition, if there is a period during the implosion when the shell "coasts," the DT fuel may decompress, raising the stagnation adiabat and the minimum kinetic energy required for ignition.

Stagnation pressure may also be sensitive to low-mode $\rho \mathrm{R}$ asymmetries in the cryogenic fuel. Possible sources for asymmetries include laserpower imbalance, intrinsic hohlraum flux asymmetry on the capsule surface, geometrical asymmetries introduced by the capsule mounting (e.g., the tent that holds the capsule, fill tube, slight offsets in the capsule location relative to the hohlraum), imperfections in the capsule fabrication and possible asymmetries of the ice layer due to thermal gradients. Asymmetries may lower the efficiency with which kinetic energy is converted to thermal energy, resulting in a lower hotspot pressure at stagnation.

Finally, higher mode instabilities or discrete perturbations, such as the fill tube or ablator surface features, may cause injection of ablation shell material into the fuel. Some 3D calculations indicate that large growth of hydrodynamic instabilities can result in jets, which divide the assembled hotspot.

\subsubsection{Status of theory and modeling}

While some progress has been made in developing 3D models of capsule implosions, much of the physics basis behind the NIF was developed using 1D and 2D radiation-hydro codes (e.g., LASNEX). These codes are intrinsically limited in their ability to model the development of the asymmetric 3D implosion structures and the myriad of possible sources for these structures. This dimensional restriction is manifested most significantly in the treatment of mix. Even though direct Numerical Simulations (DNS) of mix in 3D space and time are computationally intensive, 3D simulations are essential to correctly model its 
effects and to interpret observed experimental mix signatures.

The equation of state (EOS) data used by these codes is also based on theory, with no direct experimental validation at the high pressures achieved in ignition experiments. The EOS of the $\mathrm{CH}$ ablator has already been modified in response to ignition experiments, leading to improvements in understanding shock timing; there is likely to be similar room for improvement in the EOS of the DT fuel. The DT exists in the moderately to strongly coupled plasma regime where accurate evaluation of plasma models and material properties is difficult.

Bremsstrahlung losses or reabsorption in the high-density shell might also be more poorly understood than existing models indicate. In addition to unexpected dense fuel layer effects, the boundary between the hot core and fuel layer might contribute to enhanced thermal flow from the compressed core through non-local effects, magnetic fields, and turbulence. Separation of these effects will undoubtedly be difficult but it will allow a more quantitative understanding of energy balance between the core and fuel layer.

\subsubsection{Impact of experimental results}

Several important observations and key inferences have been made from recent cryogenic layered capsule-implosion experiments. First, a pressure at stagnation up to 150 Gbar has been inferred from a variety of observations, interpreted using a static, uniform pressure (isobaric) model for the stagnating plasma [1]. While this is a significant achievement, this pressure is still significantly lower than the point design of $\sim 300$ Gbar that is needed to initiate the burn (i.e., significant alpha particle heating). Secondly, from trends in observed neutron yields and $\mathrm{x}$-ray yields (low neutron yield, but higher $\mathrm{x}$ ray yield), there is strong evidence for mixing of ablator material into the hotspot for certain shots, further reducing the yield significantly. Thirdly, although the observed average fuel $\rho \mathrm{R}$ is about $85 \%$ of the point design, neutron-spectrometry data clearly indicate $10-20 \%$ low-mode $\rho$ R asymmetries that are generally repeated from shot to shot when hotspot mix is low. At higher levels of hotspot mix, these asymmetries are significantly smaller. The data also seems to suggest systematic pole-to-equator $\rho \mathrm{R}$ asymmetry (the $\rho \mathrm{R}$ up to $50 \%$ higher on the poles).

The $x$-ray and neutron imaging data suggest that the burning material in the hot core could be separated from the denser fuel layer by an intermediate "warm" layer. An intermediate region of "warm" moderate density material between the hot and tenuous hotspot and cold dense fuel at stagnation would contribute to lower stagnation pressure and would cause alpha deposition and x-ray absorption to occur over a larger volume, decreasing the ablation pressure from this region. Such regions are expected from heat flow with 3D features, or convection, but another explanation is that the current understanding of heat transport from hot to colder dense regions might be inadequate.

\subsection{Opportunities for Progress}

\subsubsection{What are the most important uncertainties and why?}

In this section, the uncertainties are discussed in order of importance. First, the most important uncertainty is the impact of large- and small-scale instabilities on the capsule implosion. As noted in 4.2.2, some of the large-scale perturbations can be $3 \mathrm{D}$ in nature and would have to be modeled all the way to stagnation to understand their impact. This is computationally challenging, and the impact of large-scale, 3D perturbations is therefore potentially poorly understood. On the other end of the spectrum, there can be significant growth of small-scale instabilities resulting in 3D perturbations. These perturbations can potentially create turbulent flow on scales too small to be realistically captured by hydro simulations. Capsule radiography data illustrating these limits are shown in the sidebars. The potential impact of these instabilities on the implosions is high, and some effects include the loss of implosion kinetic energy into small-scale turbulence, anomalous thermal transport of energy between the shell and the fuel, asymmetry in the assembly of the fuel, and more. Experimental progress in this area is hampered somewhat today by the lack of adequate $2 \mathrm{D}$ imaging radiography and experiments dedicated to these issues. Section 4.4.1 further 
elucidates these issues and makes an attempt to address them.

Second, the uncertainties associated with the shocks used to couple energy to the shell, and eventually to the DT gas, are also important. If too much energy is transferred to the DT gas, its adiabat will rise and the energy coupled to the stagnated fuel will not be sufficient to reach ignition. The laser pulse profiles creating the shocks are designed using modeling and feedback from liquid surrogate target experiments. This modeling could be wrong if the initial conditions are not as expected. For example, variations in the DT layer composition would change the speed of shocks through this layer. Also, the impact of material released from the inside surface of the ice layer is not assessed. These variations are not present by definition in the surrogate experiments, which do not use an ice layer. Other uncertainties in the modeling of the shocks can affect the shock timing, especially near stagnation as the combined shock begins to strengthen through convergence. Hydro codes are used to model the shocks that fundamentally do not include kinetic effects that may be present, such as non-local ion transport at the shock front, barometric diffusion, and nonMaxwellian particle distributions. Section 4.4.2 discusses these issues in more detail and how to potentially address them.

Third, uncertainties in the EOS of the DT fuel at high density, especially due to moderate coupling effects, could significantly alter late time compression of the main fuel as it begins to stagnate and reach high density. Feedback on hotspot formation will then change the configuration of the hotspot and main fuel under pressure equilibrium. This is a potentially significant uncertainty, considered further in Section 4.4.2.

Fourth, the conditions of the shell and DT gas prior to or at the start of the implosion need to be investigated carefully, as discussed in Section 4.4.3. If the DT gas density and/or temperature are different than expected, then the modeling of the implosion is fundamentally flawed. These conditions are sensitive to the vapor pressure and condensation rate of the central DT gas that is cooled 1.5 Kelvin below the triple point, in the final 30 seconds before the shot. The cooling is expected to reduce the central gas density a factor of 2 , from the value at the triple point. There is also the possibility that radial variations in the DT composition due to fractionation during the manufacture of the ice layer are arising, though this is not thought today to be a significant issue.

Another effect that could have an impact on the stagnation pressure is the incomplete elimination of the "coasting" phase (resulting from lack of understanding of hohlraum energetics and ablation dynamics), which can result in decompression of the main fuel piston prior to stagnation. To address this potential problem, the duration of peak laser power was extended until the shell begins its deceleration, but it is possible that some residual effect remains. However, it is believed that any residual effects are insignificant since the shell is at low radius and high velocity when the drive is turned off. Even allowing for a rapid fall in drive temperature, it is argued that the rarefaction from the rear surface has insufficient time to communicate with the hotspot before bang time.

During the implosion, preheating of the capsule by electrons and $x$-rays produced by the laser spots is also a concern. For example, the NIC has measured a significant number of highenergy electrons $(E>170 \mathrm{keV})$. The fuel preheat from these electrons is thought to be acceptable, but there may be significant additional heating from lower-energy electrons and non-thermal xrays that can preheat the capsule ablator, modifying the density profile of both the ablator and the shell, and again changing the modeling predictions for the shock behavior and symmetry of the drive pressure in these plasmas.

\subsection{Priority Research Directions}

\subsubsection{The origin and 3D structure of $\rho R$ asymmetries}

\section{Introduction}

Low-mode $\rho \mathrm{R}$ asymmetries, which are generally observed in cryogenic layered implosions, reduce the pressure at stagnation and the kinetic energy available to compress the fuel, which can be fatal to ignition. Indeed, the isobaric pressure model suggests that some of the available 
kinetic energy is not being coupled to the hotspot. The highest priority research direction is thus to explore how 3D asymmetries arise, evolve, and impact stagnation.

\section{Near-term improvements and approaches}

In recent years, the increasing availability of 3D simulation tools (e.g., HYDRA) has made it possible to better assess $3 \mathrm{D}$ effects computationally. These capabilities make it possible to simulate a broad range of perturbation scenarios to quantify the impact of 3D effects on critical processes and observables. Without necessarily resolving the detailed physical origin of how these instabilities might arise, we can use simulations to explore the magnitude of the perturbations required to have a measureable impact on experimental observables. For example:

- What is the impact on implosion performance of a few regions in the shell with very low $\rho R$ ?

- Can 3D effects invalidate the use of the isobaric pressure model to infer stagnation pressure?

- What level and morphology of perturbation is required to mix a significant mass of cold fuel or ablator material into the hotspot?

The goal of such simulations would be to broadly explore the types of physical processes that could be contributing to the observations and assess whether they are likely explanations. Validation experiments would be required to test the trends predicted by 3D modeling. Some example 3D simulations illustrating this approach are shown in the sidebars on 3D effects on stagnation.

There are multiple potential sources for the low-mode $\rho$ R asymmetries, such as beam imbalance, hohlraum diagnostic windows, tents, and fill tubes. In the near term, the plan is to experimentally and quantitatively assess these effects using the keyhole VISAR diagnostic that will be upgraded from its current dual point (waist and pole) to a line-imaging capability (in either polar or azimuthal directions). This would in particular allow the ability to assess capsule drive, adiabat variation versus polar and azimuthal angle, and combined with $\mathrm{x}$-ray radiography to assess how drive asymmetries affect the growth of low-mode $\rho R$ asymmetries during acceleration. In addition, these data could be complimented by improvement of the reemit ball technique to assess time-dependent drive uniformities in the foot of the laser pulse and by the examination of the emission uniformity for the modes up to 10 . These data would test some of the key modeling uncertainties associated with the 3D symmetry of the drive and of the first shock adiabat at a period when the control of the laser "quad-to-quad" power is most difficult. These data, coupled with the imaging VISAR response of the capsule, would also allow us to assess the impact and mitigation strategies for 3D hohlraum features using hydro models.

The use of the 2D ConA radiography platform planned for the summer of 2012 would allow us to measure RT growth factors of low mode drive and shock timing asymmetries to study resulting $\rho \mathrm{R}$ asymmetries during the period of high acceleration, examine the implosion symmetry in flight, and explore the presence on non-radial mass flow.

There are also non-cryogenic experiments that could explore the origin and 3D structure of $\rho R$ asymmetries. For example, using radiography to explore the growth of fill-tube-induced perturbations in an ablator during the early part of an implosion [2]. Such experiments would provide critical data and constraints for understanding how the fill tube can mix ablator material into the fuel. Implosion experiments with pre-seeded low-mode and or high-mode perturbations in the ablator, diagnosed by radiography, would likewise provide key data on instability growth in the imploding shell for validation of the physics models. Such experiments may need to artificially enhance the perturbation amplitudes above that expected in standard implosions to make the $\rho \mathrm{R}$ variations visible to radiography.

\section{New capabilities needed}

The current diagnostics suite on the NIF includes a number of high-quality diagnostics that provide a relatively comprehensive look at the plasma conditions and from which the stagnation pressure is inferred. The selection of diagnostics for the NIC was largely chosen to enable the tuning of "known unknowns" and to diagnose 
neutron-producing shots. The largest opportunities for building on this diagnostic suite that could help significantly with stagnation and burn experiments targeted at understanding sources of $\rho R$ asymmetry and its impacts are in the areas of two-dimensional x-ray radiography, $\mathrm{x}$-ray spectroscopy, and time-resolved neutron spectrometry.
$\mathrm{X}$-ray radiography is a powerful tool for diagnosing $\rho \mathrm{R}$ asymmetries. This technique has been used at numerous high energy density facilities worldwide and would be a significant enabling capability for the NIF. A sample simulated radiograph is shown in the sidebar, part 2, which illustrates the potential for radiography to provide detailed information about wavelengths and amplitudes of $\rho R$ asymmetries in an experiment.

\section{D Effects on Stagnation, Part 1}

As the capsules are hydrodynamically unstable during the implosion, $3 \mathrm{D} \rho \mathrm{R}$ and drive asymmetries are expected to develop and grow during the implosion and are generally observed in cryogenic layered implosions, which may reduce the pressure at stagnation and the kinetic energy available to compress the fuel.

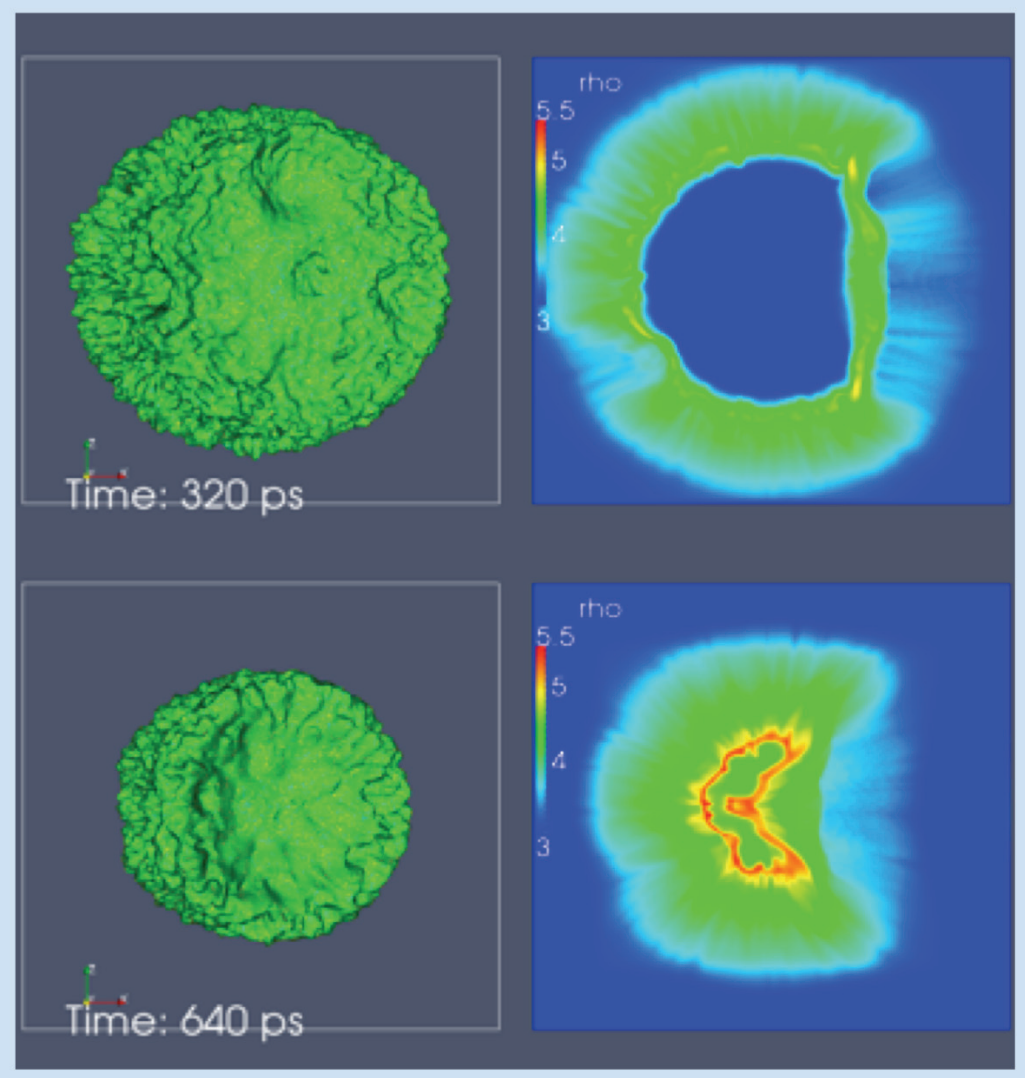

These are sample images from a 3D simulation of a cryogenic capsule implosion during the deceleration phase. The simulation was initiated with total of 13000 bubbles mapped onto the surface according to five levels of geodesic contours. Each bubble was given a random amplitude and orientation. Because each bubble converges to a different point, there is no axis of symmetry and the fuel momentum is never completely extinguished. In addition, a large amplitude single-sided push is applied from the right consistent with a capsule offset. Both the large-scale and intermediate-scale perturbations contribute to mix of cold fuel with the hot spot, which quenches the yield. The two images on the left show a surface contour of the outside of the fuel at a density of $10 \mathrm{~g} / \mathrm{cm}^{3}$. The density slices on the right illustrate the non-uniformity, which can arise in the dense fuel layer. Figures are courtesy of J. Chittenden, Imperial College. 


\section{D Effects on Stagnation, Part 2}

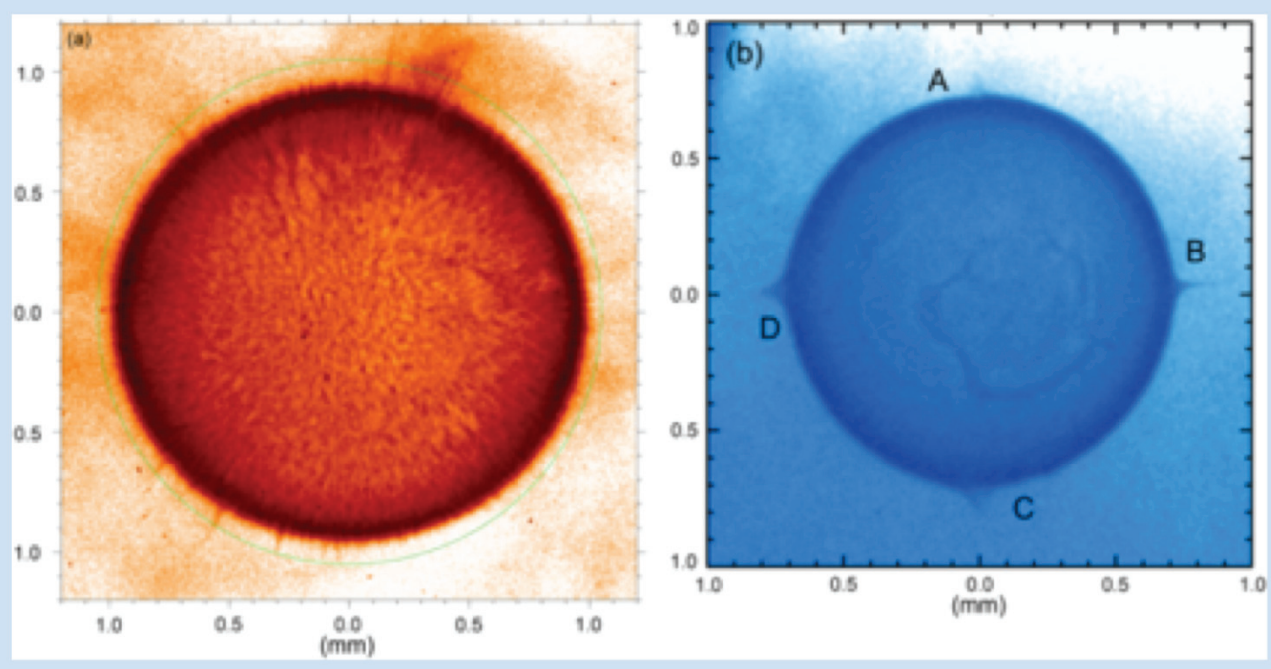

Examples of $x$-ray radiographs from indirect-drive experiments on the Sandia $Z$ facility. In the left image, the 70$\mu \mathrm{m}$-thick $\mathrm{CH}$ capsule had a 1.8- $\mu \mathrm{m}$ glass layer buried inside it, which contributed to the formation of thousands of 10- to 20- $\mu \mathrm{m}$-diameter dome defects on the capsule surface with heights $<1 \mu \mathrm{m}$ [3]. While this level of perturbation growth far exceeds anything expected on NIF, the image illustrates the power of $x$-ray radiography for providing detailed information about $\rho \mathrm{R}$ asymmetries. The image on the right is from experiments studying the growth of fill-tube-induced mass perturbations [4]. Four tubes of varying dimensions were placed at locations A, $\mathrm{B}, \mathrm{C}$, and D around the capsule, leading to varying perturbation levels. The structure in the central region of the capsule may be a perturbation induced by the formvar plastic that suspended the capsule inside of the hohlraum. Analogous experiments on the NIF could potentially provide a wealth of information.

While it may not be possible to take useful radiographs under all scenarios due to $\rho \mathrm{R}$ constraints and/or intense backgrounds, it should be possible to design experiments specifically aimed at understanding key physics with radiography as the primary diagnostic.

Obtaining sufficiently high photon energy to provide radiographic imaging of the dense fuel layer at stagnation with sufficient resolution to reveal the detailed structure requires the development of new capabilities on the NIF, such as the Advanced Radiography Capability (ARC). Whether asymmetry in the final phases is dominated by instability growth or by global asymmetries can, however, be established using in-flight radiography of the capsule with lower photon energies and spatial resolution requirements accessible using the existing facility. Such data would be invaluable in constraining theoretical models and helping to identify the dominant source of asymmetry.
The second approach is $\mathrm{X}$-ray spectroscopy. High-Z x-rays, such as $\mathrm{Kr} \mathrm{K}$-shell radiation, can be excellent surrogates for neutrons because the photon production rate scales with the square of the density in much the same way as the neutron production rate. Moreover, the detailed structure of the x-ray emission from various plasmas can change dramatically as the conditions change, which makes a spectrally resolving measurement a powerful tool for diagnosing the plasma conditions. This is illustrated in a paper by Hansen [5], which compares spectra produced by two different plasmas that otherwise produce identical neutron yields. While some of the plasma parameters can be measured with existing nuclear diagnostics on NIF, complementary x-ray spectroscopy can be used for controlled experiments isolating portions of the implosion and thus increasing the capability of the NIF. For example, the ablator is frequently doped with materials to vary its x-ray absorption efficiency, but those same materials can serve as 
spectroscopic tracers for how much ablator mass is mixing into the fuel, as has been done already on NIF [6]. Similarly, one can add Kr or Ar gas tracers to gas-filled capsule implosions (or $\mathrm{Br}$ to foam surrogate targets) to provide information about the behavior of the fuel. Tracer spectroscopy can both constrain current models for inferring the stagnation pressure and also provide information on the relative contributions of different portions of the capsule to $\rho \mathrm{R}$ asymmetries and related effects such as fuel mixing.

The third diagnostic capability is timeresolved, high-resolution neutron spectrometry for detailed measurements of the neutron spectrum. This is the next generation neutron spectrometry that has the potential to probe the time evolution of $T_{i}$, fuel $\rho R$ and kinetic effects during burn, in addition to burn history and bang time. Having time-revolved information about these implosion parameters will be essential for better addressing issues with the assembly of the hotspot and main fuel and thus greatly improve understanding in this area. The technique is based on an extension of the Magnetic Recoil Spectrometry (MRS) technique, successfully used on both OMEGA and the NIF, which utilizes a combination of a magnetic field and a streak camera to break the dispersion issue and measure the neutron spectrum with a time resolution of about $20 \mathrm{ps}$. It is anticipated that this type of system should work for neutron yields above $\sim 5 \times 10^{14}$.

In addition to these new diagnostic techniques, more conventional neutron-Time-ofFlight (nTOF) neutron spectrometers fielded on the poles are required to better diagnose the poleto-equator $\mathrm{QR}$ asymmetry often observed in cryogenically layered implosions.

\section{Long-term goals and outlook}

It is very probable that $3 \mathrm{D}$ effects on stagnation are affecting current attempts to achieve ignition. But this area of research and associated diagnostic development has considerable value independent of whether ignition is achieved in the near term. Progress in optimizing ignition platforms requires understanding the relative importance of various sources of asymmetry and mix and how these contributions are related to capsule design. Since many of the potential sources of asymmetry are fully $3 \mathrm{D}$ in nature, the long-term goal should be to develop an experimental database that can be used to validate and improve modeling capabilities as they mature, particularly since robust 3D computing is still in its infancy. While there have been excellent experiments done on smaller-scale facilities for precisely this purpose, the reality is that the modeling codes contain adjustable parameters tuned to those experiments (e.g., flux limiters), and it is not known whether those parameters have the same values at the conditions found at NIF.

A multi-year plan of experiments and diagnostic implementation should be developed in support of this research direction. Time should be allocated to this effort each year and maintained on the schedule regardless of progress toward ignition. The ordering of experiments will depend on the ability to bring the corresponding capabilities to bear. Early experiments should focus on relatively easily measured sources of yield degradation, such as understanding the effect of the fill tube (low-mode asymmetry) or the impact of increasing levels of gas dopants in gas-filled capsule (mix). As high-resolution radiography diagnostics become available, experiments looking at high-mode instability growth and asymmetry will become possible. Concurrently, 3D modeling efforts should be pushed to assess the relative importance of the various possible sources of $\rho R$ asymmetry. These should be a combination of coarsely- and finelyresolved simulations to get a handle on the issues.

\subsubsection{In-flight characteristics of the DT fuel}

\section{Introduction}

The configuration of the hotspot and surrounding cold fuel at stagnation is sensitive to the in-flight density profile of the high-density DT shell. Achieving ignition depends on compressing the fuel by a factor of 30 , on a low adiabat at high density, while maintaining a sharp boundary between the cold fuel and the hot gas. If fuel is prematurely introduced into the nascent hotspot, the stagnation pressure is reduced. Preheat, EOS uncertainties, coasting, and additional shocks are 
effects that can contribute to a less than optimal fuel configuration.

\section{Near-term improvements and approaches to theory and modeling}

Near-term improvements to modeling can help by assessing the sensitivity of radiationhydro simulations to unquantified physics, exploring kinetic effects absent from the standard fluid modeling approaches, or improving the analysis of observations.

Simulations to assess sensitivities are useful where the cause of any performance discrepancy has not yet been identified or where it is difficult to directly quantify aspects of the implosion performance. Given that it takes time to develop and commission additional experimental platforms, sensitivity studies represent a near-term measure to assess the likely importance of hypotheses. For example, simulations could be performed to study the impact of different preheat levels (from both hot electron and non-thermal xray sources) on the calculated shell density profile and subsequent fuel assembly. Sensitivity to preheat levels may have consequences for shock timing requirements.

While it may be pragmatic to adjust the simulations (for example, by tuning the DT EOS) to match observed stagnation conditions, there is no substitute for better physics models based on improved theory or models informed by direct measurements. For long-term fidelity of the simulations, it is imperative to improve the EOS models for both the DT and ablator, as well as radiative and transport models that affect ablation pressure, as these are fundamental to the fidelity of the design tools. Improved material EOS and radiative models for both DT and ablator materials informed by direct measurements and better theory should be pursued. Meanwhile, tuning the simulations to bring them in line with observed stagnation properties could highlight the sensitivity of the simulations to uncertainties in the materials properties, and lead to modified code predictions that could change the required shock timing for ignition. If timing requirements were found to be significantly different, this study could be used as a basis for performing an empirical scan of shock timing.
Kinetic modeling of convergent shock propagation through the capsule would allow the effect of additional physics not present in the hydrocodes to be assessed. In particular, the extent and impact of non-local ion transport on the shock propagation and on the conversion of kinetic to thermal energy at the center of convergence must be investigated, as the longer range energetic ions could cause the shock front to be spatially smeared. Self-generated electric fields at the shock front may also produce ion species fractionation through barodiffusion, although a full assessment of this would require appropriate collision models to correctly capture relaxation of the ion species ratio behind the shock. In addition, the development of non-Maxwellian distributions within the shock front could occur through reduced rates of electron-ion relaxation at the high-energy tail of the distribution.

These same kinetic effects may also play a role in the formation of the hotspot, with its return shock, and the subsequent transport of ions and reaction products out of the hotspot. This is important as escape of energetic ions from the hotspot was also suggested as a mechanism by which the DT fusion reactivity could be significantly reduced, since the reactivity is dominated by the tail of the ion distribution in current NIC experiments. Kinetic simulations could be used to study the balance between ion loss and ion-ion relaxation.

Experimental studies on the NIF and OMEGA can systematically study the effects of varying the duration of the $4^{\text {th }}$ pulse on coasting. Simulations do not predict the extent of coasting observed experimentally. In part, this is due to the lower hohlraum-capsule coupling efficiency. While progress has been made in eliminating coasting by extending the $4^{\text {th }}$ pulse, a more detailed study should be used to parameterize the effect and help understand its origin. It would be useful as well to further extend the $4^{\text {th }}$ pulse to confirm empirically that any residual coasting has been suppressed. An empirical study of stagnation conditions as a function of the launch time of the $4^{\text {th }}$ shock should also be undertaken. Comparing trends in the data with those expected from simulations would highlight any disagreement and would help guide corrections and the identification of missing physics. For example, the empirical trend would 
establish whether hotspot conditions could be improved by optimization. A platform to perform DT-layered keyhole shock timing experiments should be established to measure the impact of inner surface release, which does not occur in the existing surrogate liquid-Deuterium keyhole target. Other improvements that could be pursued include spectrally resolved preheat characterization via improved resolution of nonthermal x-rays and measurements of hot electrons at $\mathrm{E}<170 \mathrm{keV}$ and studies of the scaling of hotspot pressure with implosion velocity. Theory predicts that $\mathrm{P} \sim \mathrm{v}^{3}$. Any deviation from this trend may be indicative of additional physics effects.

\section{New capabilities needed}

New capabilities are required to support the suggested modeling and experiments. The current diagnostics suite on the NIF does not include a DT-layered keyhole platform, which is required for performing shock timing experiments that will address questions about the release from the DT inner surface. A modified version of the target, which includes DT ice on the surface of a roundended re-entrant cone could be used to study the stagnation shock as the imploding ice layer impacts on the central ice.

Improved $\mathrm{x}$-ray preheat characterization would require a spectrometer mounted to view the hohlraum wall. The Dante diagnostic currently in use has insufficient channels in the relevant $\mathrm{x}$-ray region $(\sim 2 \mathrm{keV})$ of the spectrum to adequately constrain the non-thermal drive. To measure hot electron flux at lower energies than currently possible will require a new diagnostic platform.

Kinetic modeling of effects such as ion species separation by barodiffusion, the impact of non-local ion transport on shock stagnation and electron-ion relaxation will require appropriate collision models for kinetic codes, valid at the high densities encountered during stagnation. This may be beyond the current state of the art.

\section{Long-term goals and outlook}

For long-term fidelity of the simulations, it is imperative to improve the EOS, radiative, and transport models, including possible kinetic effects, that determine ablation pressure, as these are fundamental to the fidelity of the design tools.
Issues such as "coasting" or the development of a deleterious 5th shock are ultimately tied to the accuracy of our EOS and radiative models, as these govern how the radiation is produced, how it is transported, and how the ablator and DT respond to it. Improved theory and models for the EOS and radiative properties of the hohlraum, DT, and ablator, validated by direct experiments, should be pursued to improve the long-term fidelity of simulations.

\subsubsection{Probing energy balance at stagnation}

\section{Introduction}

The simplicity of the consequences drawn from the picture described in Section 4.2.1 lead to equally straightforward suggestions for progress based on experiments and simulation or simulation of the expected properties of the implosion conditions at stagnation [7]. These directions fall into three broad categories: an assessment of early material release into the hot core, an investigation of unconventional heat transport, and unanticipated high-density opacity effects.

\section{Near-term improvements and approaches}

Pertaining to the first category, experiments to directly measure fuel-ablator mix at early times well before the implosion has undergone significant acceleration should be designed and fielded, examining both early shock release and unexpectedly large hydrodynamic instabilities. These experiments need to be supported by appropriate analytical models and more fully developed 3D simulations (discussed in Sec. 4.2.2), which will directly assess the effects of surface perturbations seeded by the measured roughness spectrum. It is anticipated that the relevant experiments should be designed to be much more limited in scope than a full capsule implosion, so that different effects can be more readily diagnosed and simulated. It is also expected that existing nuclear and $\mathrm{x}$-ray techniques, or extensions of these techniques, will be used to diagnose mix and stagnation pressure. The expected experimental deliverable will be a quantitative estimate of any cold material released prior to stagnation into the eventual hot core. 
For the second category, experiments are needed to provide a more detailed understanding of the heat transport from the hot core to colder, dense regions at stagnation. Both NIF and OMEGA experiments, which might require cryogenic layered capsules, should be fielded to examine this potential issue. The main thrust of the proposed experiments will be the development of diagnostics that could directly measure the spatial profiles of the plasma conditions in the hot core and dense fuel. Both x-ray emission and charged particle nuclear tracers should be used by varying the tracer implantation depth to probe different density and temperature conditions. Dedicated simulations supporting these experiments should also provide some insights into the magnitude of the transport mechanism. In particular, different simulation platforms already exist that should be exercised to evaluate nonlocal effects, magnetic field strength, and turbulent flow (Tipton, CALE). The expected deliverable will be an accurate transport model valid under stagnation conditions.

Finally, the energy balance in the stagnated assembly should be directly addressed. The mechanical (PV), thermal (kT), and kinetic $\left(\mathrm{MV}^{2}\right)$ contributions at stagnation must balance the experimentally determined maximum kinetic energy available to the remaining ablator and fuel, accounting for radiative loss and alpha heating. Critical to this accounting is an understanding of energy lost to radiation. Thus, experiments designed to quantify the opacity of the dense fuel and ablator material are necessary. If the conventional treatment of radiation transport is correct in this density regime, then any energy deficit must arise from 3D asymmetric implosion effects. This determination would have an immediate, profound impact upon future experiments, since experimental effort would be refocused to eliminate these effects.

\subsection{Conclusions}

Analysis of the cryogenically layered capsule implosions performed to date indicate, first and foremost, that even when implosion modeling is tuned to reproduce shock timing and implosionvelocity measurements, the observed stagnation pressure of the hotspot and cold fuel are significantly lower than predicted. Since an understanding of the underlying physics of this pressure deficit is essential to achieving ignition, this chapter discusses the issues with the models used to infer the stagnation properties and outlined priority research directions that should shed some light on this issue. The high priority research directions are:

1. The origin and 3D structure of $\rho R$ asymmetries.

2. In-flight characteristics of the DT fuel.

3. Probing energy balance at stagnation.

\subsection{References}

1. P. Springer et al., Proceedings Inertial Fusion Sciences Applications (2011).

2. G. R. Bennett et al., Phys. Rev. Lett. 99, 205003 (2007).

3. M.E. Cuneo et al., IEEE Trans. Plasma Sci. 39, 2412 (2011).

4. G.R. Bennett et al., Phys. Rev. Lett. 99, 205003 (2007).

5. S. Hansen, Phys. Plasmas 19, 056312 (2012)

6. S. Regan et al., Phys. Plasmas 19,056307 (2012).

7. P. Clark Souers: Hydrogen Properties for Fusion Energy (1986) University of California Press. 


\subsection{PANEL 5 - HED PROPERTIES AND PROCESSES}

\subsection{Introduction}

Optimizing implosions for igniting and propagating thermonuclear burn at the NIF requires understanding HED states over an unprecedented range of conditions. To date, none of the physical models integral to ignition simulations are benchmarked against data over most of the ignition implosion regime. In this report, results from the NIC are used to guide and help prioritize a plan to benchmark and improve key physical models used for ICF calculations, including EOS; opacities, both in LTE and nonnon-LTE cases; transport quantities (thermal conduction, electron-ion equilibration, ion stopping power, viscosity), kinetics, electric and magnetic field generation, nuclear reactions, and the interplay between these areas of physics in an HED environment.

In an ICF implosion, the initial laser energy is transferred to the fuel through a complex chain of energy conversion processes. When laser energy of $\sim 1.6 \mathrm{MJ}$ is incident on an ignition hohlraum, it is absorbed collisionally and reemitted as $\mathrm{x}$ rays with approximately $80 \%$ efficiency. Losses include back-scattered light and the acceleration of non-thermal, high-energy electrons that can preheat the capsule, making compression more difficult. The transport of $x$-ray energy from the hohlraum wall to the capsule results in $\sim 140 \mathrm{~kJ}$ of energy at the ablation front of the ignition capsule. Modeling the emission, absorption and propagation of $\mathrm{x}$-rays in the hohlraum requires accurate LTE and non-LTE opacities, and EOSs for regions of both pure and mixed materials. Understanding the effects of "hot-electrons" requires an understanding of electron transport in a wide range of hohlraum plasma states. Ablation of the capsule surface, which drives the implosion, depends on x-ray absorption and reemission, the ionization state and sound speed of matter near the ablation front. These quantities control the "rocket effect" that accelerates the shell to a kinetic energy of $\sim 20 \mathrm{~kJ}$.

During the early stages of the implosion, the $\mathrm{x}$-ray energy increases with time, driving a series of shock waves through the ablator and into the DT fuel. The cold DT fuel is compressed to a dense, Fermi-degenerate layer on the interior of the ablator shell, and then accelerates to peak velocities of near $370 \mathrm{~km} / \mathrm{s}$ at $10-20 \mathrm{~g} / \mathrm{cm}^{3}$ and $\sim 10 \mathrm{eV}$. During this phase, the stability of the fuel-ablator interface depends on the density profile of the ablator and fuel, which are set by the $\mathrm{x}$-ray absorption, thermal transport, and EOS. At stagnation, the hotspot must reach a density of $100 \mathrm{~g} / \mathrm{cm}^{3}$, an aerial density of $300 \mathrm{mg} / \mathrm{cm}^{3}$, and a temperature of $5 \mathrm{keV}$, while the dense fuel surrounding it must reach $1 \mathrm{~kg} / \mathrm{cm}^{3}$ at just $50 \mathrm{eV}$. At these extreme conditions, there is little data to constrain physics models. Hotspot initiation is a race between energy production and transport, including nuclear reaction rates, thermal transport from the hotspot into the cold fuel, electron-ion equilibration, ion stopping power, and $\mathrm{x}$-ray emission and reabsorption.

Of course many other physical processes may be important to optimizing ignition implosions, such as ion kinetics, large electric or magnetic fields, viscosity, etc. While significant effort has gone into developing the materials models used in designing ICF capsules, most are untested in the extreme conditions accessed by the ICF implosion. This situation is addressed below in four PRDs covering EOS, opacity (LTE and nonLTE), transport and kinetics, and nuclear science.

\subsection{Status of the Physics}

Equation of state

EOS controls the hydrodynamic response of materials involved in the ICF implosion. The DT fuel is compressed to more than $1.5 \mathrm{~kg} / \mathrm{cm}^{3}$, three orders of magnitude higher that the density at which benchmarking data currently exist. Given the many recent discoveries in high-pressure science at compressions of only a factor of a few, this new NIF regime of ultra-high density matter is ripe for discovery [1]. Strong shocks in the lowdensity DT gas together with the release physics of dense states of hydrogen help determine the hotspot entropy, a crucial parameter controlling hotspot assembly.

The EOS of the ablator under compression, release, and in hot expanded states is also integral to optimizing the implosion efficiency. The hot 
expanded states are often not in LTE, an effect that is not traditionally included in detailed EOS models. Errors in the ablator EOS can lead to significant discrepancies between hydrodynamic simulations and capsule implosion data. Some of these differences (shock timing, implosion velocity...) can be compensated for using multipliers on the radiation flux driving the implosion, but this procedure can push other material states that are not measured (i.e., final hotspot assembly, dense confining fuel assembly) further from simulation.

Equations of state integral to ICF simulations have typically been represented by tables based on quotidian EOS (QEOS) [2], a Thomas-Fermi model tuned to match data. Ab initio calculations for hydrogen, and, to a more limited extent for $\mathrm{CH}, \mathrm{Be}$ and diamond ablators, have recently been performed over many of the regions relevant to ignition. In cases where no data exists, $a b$ initio calculations are being used to constrain the QEOS-based tables. The methods used, density function theory (DFT) and path integral Monte Carlo (PIMC), though based on first principles, use uncontrolled approximations. DFT calculations are based on an exchange-correlation functional that is relatively untested in the density-temperature regime of interest. The PIMC treats degeneracy effects under the fixednode approximation. Also, statistical errors have to be considered. For example, when the degeneracy parameter is of the order 0.1, PIMC calculations give error bars of the order of $10 \%$. In DFT calculations, the ions are treated classically even at very high density and low temperature, where zero point quantum effects can make a nonnegligible contribution. Practical

implementations of DFT are also typically limited to temperatures below $10 \mathrm{eV}$ or so. Despite these limitations, these $a b$ initio theoretical methods offer the best approach we have at present for determining the EOS of hydrogen under conditions relevant to ICF implosions. At higher temperatures, a model based on solutions to the Dirac equation for an average ion-in-jellium ("Purgatorio" code) [3] can be used to improve on the Thomas-Fermi physics.

Problems with standard models of the EOS of the $\mathrm{CH}$ ablator have arisen most visibly in the NIF keyhole shock timing experiments, which have revealed significant deviations from predictions [4]. Each new ablator data set has led to a new

Phase Diagrams for CH Ablator and DT Fuel

Summary of pressure-density states of $\mathrm{CH}$ ablators (left) and temperature-density states for the hydrogen fuel (right). The CH figure shows the shock Hugoniot (pink dashed curve), the cold curve (blue solid line), and the trajectory for one particular $\mathrm{CH}$ zone a few microns from the DT-CH interface in an ignition simulation. The hydrogen figure shows the compression path for a DT zone again a few microns from the DT-CH interface. The only data constraining these EOS models is on the Hugoniot to $10 \mathrm{Mbar}$ for $\mathrm{CH}$ and $3 \mathrm{Mbar}$ for $\mathrm{D}_{2}$.

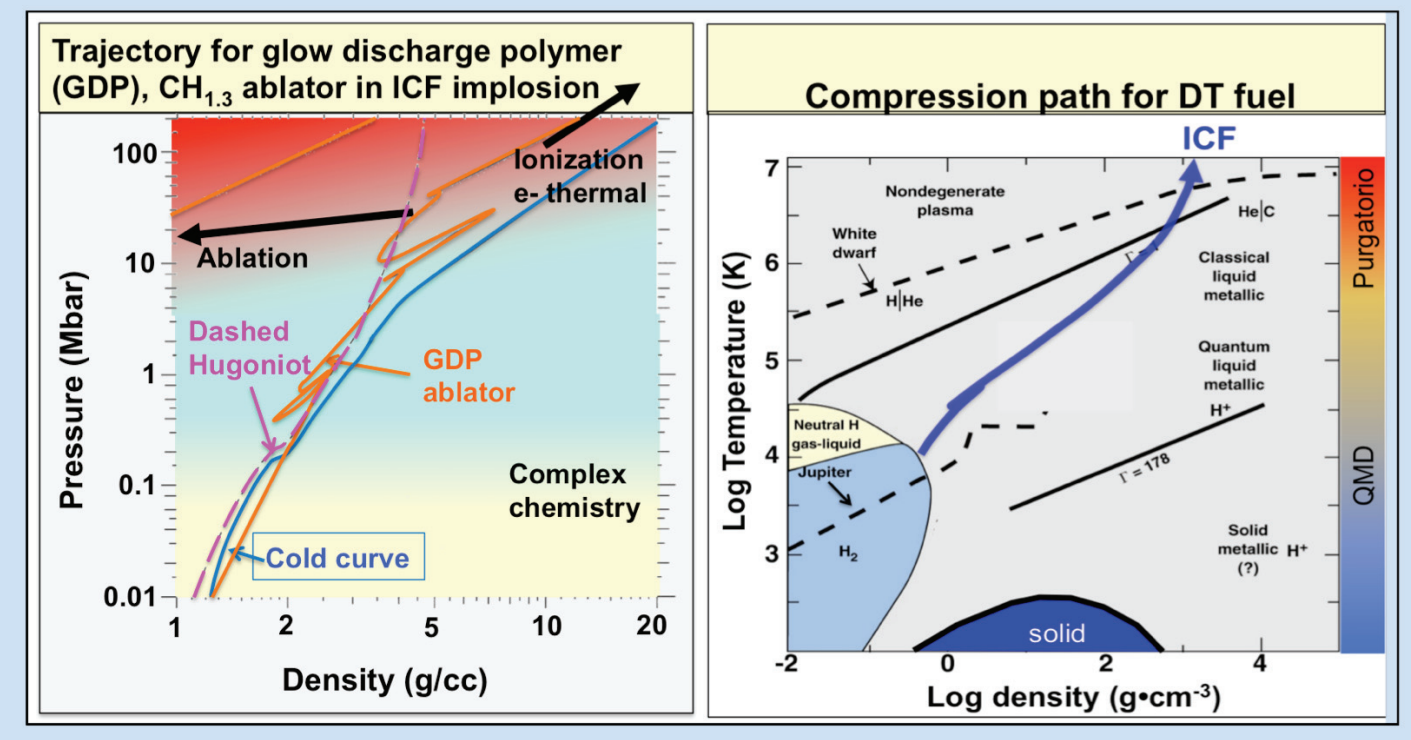

Panel 5-HED Properties and Processes 
ablator EOS model producing important changes to ignition designs. For example, changing the CH EOS to match single-shock Hugoniot [5] and release [4] data below 10 Mbar and changing the model at high temperature from Thomas-Fermi to Purgatorio, led to a change in the flux multiplier needed to match the strength and timing of the second shock from $\sim 50$ to $20 \%$. Almost any highpressure, high-temperature data on potential ablator materials is important for improving the predictive power of implosion simulations. This includes Hugoniot data for $\mathrm{CH}, \mathrm{Be}, \mathrm{C}, \mathrm{B} 4 \mathrm{C}, \mathrm{Al}$, and other materials to $>100 \mathrm{Mbar}$, ramp compression data to $100 \mathrm{Mbar}$, and release data from compressed and heated conditions.
Moreover, the DT EOS is still not well known in a number of regions important for ICF implosions:

1) The compression path to $1.5 \mathrm{~kg} / \mathrm{cm}^{3}, 2$ ) release paths from multiply shocked hydrogen, and finally 3 ) the gas shock dynamics. For example, scientists at CEA have developed a new DT EOS model which results in significantly different performance in simulations compared to the LLNL DT EOS [6].

There are a number of experimental approaches to obtaining more EOS data. Combining precompression in a diamond anvil cell (DAC) with laser shock compression can extend DT EOS data off the principal Hugoniot

\section{High-Pressure Equation of State Measurements}

Planar techniques for both shock and ramp waves have been routinely used and published in the literature. Convergent techniques shown below provide a new way to collect data into the many Gbar regime. Experimental setup is very close to the convergent ablator experiments routinely used to collect implosion velocity and remaining mass. In these experiments, both shock velocity and density are collected versus radius to provide an EOS trajectory for a convergent Hugoniot.

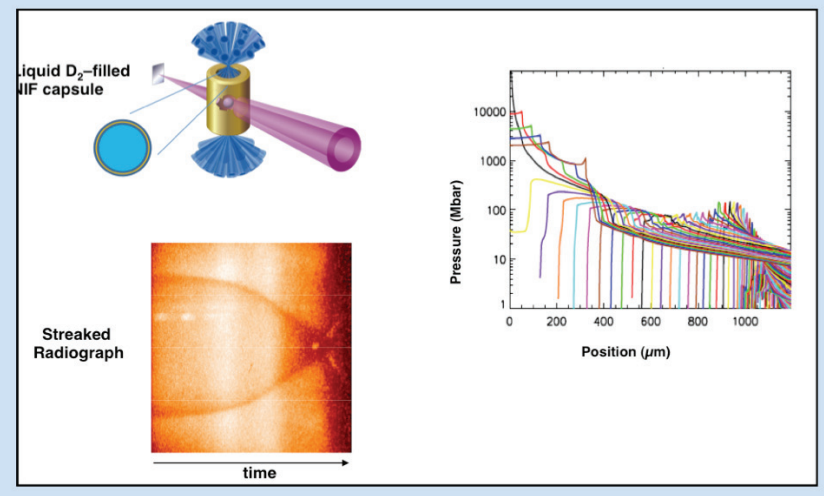

The release path for heated states can be mapped out with recently developed techniques that infer the EOS from the measured expansion of shocked or isochorically heated matter [7]. The figure below shows an experiment in which a thin copper foil was irradiated with a few-ps pulse of protons and its expansion was observed using x-ray backlighting. This method may be useful in understanding the EOS of the hot hohlraum wall material and the ablated material that comprises the corona around the capsule. The image on the right shows the setup for collecting data for hot expanded states. The image on the right shows a streaked side-on x-ray shadow of the foil before and after proton irradiation. The rightmost radiograph shows a faster streak record showing the expansion of the foil shadow over an 800-ps time.
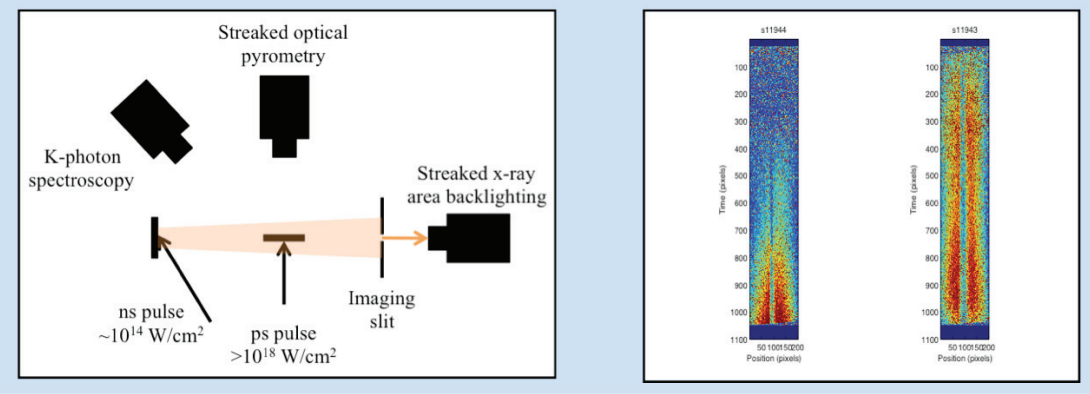

Panel 5-HED Properties and Processes 
[8]. A systematic use of this technique will produces a set of Hugoniot curves covering a broad phase space, and provide accurate EOS data over the density range of $0.06 \mathrm{~g} / \mathrm{cm}^{3}$ to $4 \mathrm{~g} / \mathrm{cm}^{3}$ and temperatures up to $10 \mathrm{eV}$. Multi-shock experiments in cryo- or DAC targets could be used to achieve the $10 \mathrm{~g} / \mathrm{cm}^{3}, 10-\mathrm{eV}$ range. Higher density states can be achieved in convergent geometries with radiography and Thomson scattering. Finally, keyhole experiments can be used to characterize DT under conditions corresponding to the interior of an imploding ignition capsule. This requires formation of $\mathrm{DD}$ and DT solid layers that have 'free surfaces' abutting a gas in a geometry that affords diagnostic access.

Simulations based on DFT have been carried out for DT and carbon-hydrogen systems similar to the $\mathrm{CH}$ ablator used in current implosion experiments. These suggest that the $\mathrm{CH}$ mixture is not ideal at high densities. Under second shock conditions, the carbon-carbon bonding seems to persist, with hydrogen moving like a fluid in the carbon network. At higher densities, there appears to be some evidence for separation between hydrogen and carbon. A systematic series of calculations for $\mathrm{CH}$ and all other ablator candidates integrating DFT, PIMC and Purgatorio needs to be done and integrated into EOS tables.

The EOS of hot $\mathrm{Au}$ or $\mathrm{U}$ is required to model the response of the hohlraum. This is important for modeling the expansion of the hohlraum during intense laser irradiation and the closure of the LEH. It can also affect the absorption of radiation by the ablated capsule material. NonLTE EOS modeling is desirable to maintain consistency between the EOS and the opacity. Accurate modeling of non-LTE physics is a complicated problem. Simplified EOS models that rely only on the effective ionization of the material may not be adequate. Models may need to account for the way in which ionization state changes modify the interatomic interactions in the material, thereby changing both ionic and electronic EOS components

\section{Opacity}

The "opacity" of a material controls its interaction with photons, including emission, absorption, and transport. It depends on the detailed atomic structure of matter and the distribution of population in accessible atomic states. In some cases, it can be approximated satisfactorily by simple simulations and represented by tabulated parameters. But in general, it is a complex, computationally intensive problem, and soluble treatments can entail significant shortfalls in accuracy. Predictive ICF simulations require accurate opacity modeling, since in many stages of the implosion, photons are the dominant mediator of the energy transfer. In some regimes, the complex interaction between the radiation and matter can lead to nonlinear feedback, strongly influencing the hydrodynamic path taken by the target. The opacity of the hohlraum is important in determining the $\mathrm{x}$-ray drive, and the ablator opacity helps determine the rocket efficiency. In the imploded DT core, opacity plays a role in deleterious cooling. Understanding opacities is also crucial to interpreting many of the diagnostics fielded in ignition experiments.

The accuracy of the tabulated opacities in current use may well be insufficient for predictive modeling. For example, most LTE opacity models use very similar basic assumptions to account for density effects in HED environments, assumptions that are now being called into question. Moreover, the simplifying assumption of LTE does not hold over much of the parameter space accessed at the hohlraum wall, hotexpanded ablator, and mid-Z material mixed into the hotspot. Non-LTE models are more difficult to implement in simulations than LTE opacities, but are required to account for effects such as a nonPlanckian incident radiation spectrum, fluorescence, and non-Maxwellian electron distributions (e.g., hot electrons generated by LPI). Finally, it is not clear how sensitive the hydrodynamic simulations are to the numerical (e.g., number of radiation groups) and algorithmic (diffusion/IMC/SN) choices for the treatment of self-consistent radiation transport.

Very few experimental data constrain the methodology for opacity calculations, and no opacity data exist for ablator or hohlraum materials at the most extreme of the implosion conditions. From NIC experiments, three clear observations motivate efforts to improve opacities. First, an improvement in modeling 
non-LTE atomic physics of the hot blow-off Au of NIC hohlraums was important for explaining through the "high flux model" - early NIC experiments [9]. However, high-spectralresolution measurements of the Au emitted x-ray spectra are needed to provide direct and stringent tests of these models. Second, worse than expected rocket efficiency of $\mathrm{CH}$ ablators may be connected with inadequate modeling of the hohlraum spectrum and or radiation absorption along the path to the ablation front. Other questions that persist also suggest limitations in current radiation production and transport modeling, such as the magnitude of difference in performance between $\mathrm{Ge}$ - and $\mathrm{Si}$-doped ablators. Finally, while spectroscopy of the shell dopant is a promising mix diagnostic, uncertainties in atomic physics limit the interpretation of the hotspot conditions.

Almost any accurate opacity or emissivity data on NIF or other HED facilities in relevant regimes will improve our opacity modeling. Even a systematic series of measurements of shock velocity and burn through times near $300 \mathrm{eV}$ at NIF will help constrain radiation transport models.

Modifications to the ionization potential depression modeling could have an impact on a NIF capsule in a number of ways. First, it modifies the charge state distribution and hence the opacity of the plasma. Second, it means that the $\mathrm{K}$-edge of the $\mathrm{C}$ ions within the plastic shell is unlikely to be at the photon energies predicted by the codes. Third, it has significance for our ability to diagnose implosion conditions using emission from high-lying states of dopants. It is thus desirable that further experimental effort be deployed to re-evaluate some of the fundamental, and largely untested, first-order assumptions that lie within the complex opacity codes.

Finally, advanced calculations that integrate better atomic physics models and self consistency between opacity, EOS and transport and that seamlessly transition from LTE to non-LTE depending on self-consistent determination of equilibrium, will be an important step in improving our predictive capability of indirectdrive experiments.

Transport
An accurate description of the mechanisms through which energy is transported to and redistributed within the imploded core is key to understanding the path towards fusion ignition. In the hotspot, ions, electrons and radiation are characterized by different energy distributions and equilibrate through collisional relaxation. Energy produced in the hotspot from both compression and DT fusion reactions generates non-thermal components in the velocity distributions. In addition to energy equilibration, energy is transferred from the hot core to the surrounding cold fuel through electronic heat conduction and radiation.

In a one-dimensional (1D) picture, all the time constants for energy transfer/production are comparable (i.e., electron-ion equilibration, ion stopping, electron conduction, confinement, bremsstrahlung, fusion). Beyond this 1D picture, three-dimensional asymmetries created by hydrodynamic instabilities during the compression phase will exist at several interfaces, including the hotspot boundary. These asymmetries may, in turn, lead to a turbulent state in the plasma, further redistributing energy and possibly generating magnetic fields. Depending on the details of the turbulent fluid motions and associated electromagnetic fields, one might expect to find significant alterations to the usual transport coefficients found in non-magnetized plasmas.

The energy transfer production processes help set a characteristic timescale of the implosion. The overall bootstrap heating time scale depends on the fusion reaction rate, electron-ion equilibration rate, and DT-alpha stopping time. In the NIC point design, these quantities are all of the order of picoseconds. Similarly, the cooling time scale is determined by the electron heat conduction and bremsstrahlung emission power, both $\sim 10 \mathrm{ps}$. Both of these time scales are comparable to the hydrodynamic disassembly time of several tens of ps in 1D - likely less in the presence of mix.

Many transport models used in the codes (electron-ion equilibration, stopping power) were developed in the 1980s [10]. The radiationhydrodynamic models currently used to design the NIC targets are based on a fluid-level of description for the plasma. This level of 
description assumes that the plasma is quasineutral, in LTE, and characterized by a Maxwellian distribution (i.e., one or two temperature parameters). Molecular dynamics computations of DT plasmas at fusion conditions can put the electron-ion collisions, radiation emission and absorption and the nuclear reaction processes all on an equivalent footing to provide an important platform to test several approximations used in the radiationhydrodynamic computations.

Almost no transport data exist to benchmark transport models in relevant ICF regimes. There are some optical reflectivity data and electrical conductivity data up to $2 \mathrm{~g} / \mathrm{cm}^{3}$ and a few thousand kelvin. These data differ from models by more than an order of magnitude, a figure that should be compared with sensitivity calculations, which show that conductivity variations of $3 \mathrm{x}$ at $10 \mathrm{~g} / \mathrm{cm}^{3}$ and $10-\mathrm{eV}$ change significantly the hydrodynamic behavior of ignition simulations. There are some discrepancies between NIC data and simulations, which could potentially be linked to an inadequacy in our transport models. For example, the measured x-ray burn width, $t_{x}$, and nuclear burn width, $t_{n}$, are both larger than calculated and often $t_{x}<t_{n}$ in measurements, while calculations suggest $t_{x}>t_{n}$. This discrepancy may be the result of our inability to estimate heat transfer among the hotspot constituents. A second example is that the larger than expected mix fraction in the hotspot can potentially be due to incorrect estimates of heat flow across interfaces in the capsule. Finally, our inability to correctly estimate yield may be linked to our inability to calculate heat transfer and production in the hotspot.

The need for validation of transport models can be summarized in four questions:

Is the assumption of thermalized Maxwellian distributions valid? The assumption of Maxwellian (or Fermi) distributions underpins most of the formulas for transport-related quantities in the hotspot such as the formulas for relaxation rates, electron-ion coupling, nuclear reactivity and stopping powers. The basic question as to whether the Maxwellian assumption is truly valid needs to be demonstrated.
Are electron-ion equilibration rates correctly modeled and can they be validated by experiments? Recent experiments [11] investigating this issue have suggested that the equilibration rates are slower than computed with the simple Landau-Spitzer expressions. More detailed theoretical studies are taking into account collective effects, but there remains a need to validate these theories.

Can current stopping power formulas be validated by experiments in dense plasmas? Currently, no strong experimental tests of stopping powers in plasmas have been reported. Formulas accounting for plasma effects have been developed, but they need testing.

Can current conductivity models be validated by experiment? The current rad-hydro designs exhibit sensitivity to the thermal conductivity of the compressed fuel, strongly affecting the stability of the capsule to Rayleigh-Taylor instabilities. Recent conductivity models have yielded differing results, thus, an experimental test would be useful to validate the models and understand this sensitivity better.

\section{Kinetics}

Since NIC radiation-hydrodynamics codes are largely based on an average-ion ("atom"), Eulerbased fluid description, they cannot model selfgenerated shock-driven electric fields in imploding capsules, species separation in a mixture such as $\mathrm{CH}$ or DT, non-standard binary species diffusion driven by pressure gradients, electric fields and temperature gradients, the morphology of plasma shocks or effects that depopulate the high-energy tail of a particle distribution. Modeling these phenomenon require new methods and techniques such as multi-fluid or PIC approaches. Existing data with exploding pushers on the OMEGA laser facility show a variety of anomalies that may be related to species separation and kinetic phenomena [12]. The PIC/multi-fluid simulation tool - Large Scale Plasma (LSP) - shows promise as a mainline tool to help describe and understand such potential phenomena in ignition experiments.

Several strictly kinetic phenomena may affect hot spot formation and ignition thresholds. Haines has recently proposed that energetic electrons with 
mean free paths on the order of the hotspot spatial scale can deposit energy in the inner layers of the cold dense main fuel [13]. This could critically affect the mass ablation rate of the DT fuel, which produces the majority of the final hot spot mass. The kinetic structure of the return shock as it outwardly transits the gaseous (inner) region of the fuel has a low Mach number $(<2)$, leading to a significantly increased shock width and ion and electron mean free paths that are not well described by fluid-based methods. Such kinetic ions and electrons could lead to anomalous diffusion and energy transport processes that affect subsequent hot spot formation.

A number of species-separation effects in ICF capsules have been predicted, including (1) deuterium and tritium fuel ion separation during shock passage due to differing charge-to-mass ratios; (2) reduced rocket efficiency in mixed species ablators, e.g. $\mathrm{CH}_{1.3}$; and (3) reduced x-ray drive transmission to the ablation front in a spherically converging geometry. These effects are based on simple physical models and have not yet been well validated by simulations. The first effect results from the strong intrinsic electric field and pressure gradient across a shock front that transiently creates a drift speed between the deuterium and tritium ions. Although the degree of physical separation is not significant, estimates of the degree of frictional heating generated in the cold dense main fuel as the deuterium moves through the tritium across a shock front thickness suggest on the order of 100 Joules are deposited. This level of predicted auxiliary heating is marginally tolerable for achieving ignition according to 1-D radiation-hydrodynamics simulations. The second effect is a reduced rocket efficiency for a mixed species ablator such as $\mathrm{CH}$ of $5-10 \%$ as compared to a single-species rocket efficiency (for carbon ionization state of 4-6). The third effect arises from a combination of species separation and spherical geometry in effectively increasing the opacity of the blow-off plasma to incident drive $\mathrm{x}$ rays. Species separation in a $\mathrm{CH}_{1.3}$ ablator leads to a hydrogen-rich plasma at large radii, and a carbon rich plasma closer to the ablation front [14]. The absorption of drive $x$ rays is proportional to the areal density of the intervening ablated plasma, and the spherical geometry leads to locally higher areal density at smaller radii where the carbon preferentially resides. Analytical estimates of this effect suggest a several percent reduction in mass ablation rate from the combined effect of spherical converging geometry and species separation.

Almost no data have been collected to benchmark these kinetic phenomenon. Early experiments by Rygg showed that for OMEGA implosions, significant species separation takes place and significant electric fields result in the implosion phase of the capsule [12]. While estimates suggest such phenomenon should be significantly reduced in ignition implosions due to the different collisional regimes, quantitative understanding of such effects do not exist. Given the integral nature of NIC implosions, it is difficult to isolate any particular effects due to kinetics. However, both the poor rocket efficiency of $\mathrm{CH}$ ablators and the higher than expected hotspot adiabat could be linked to kinetic effects.

Kinetic and species separation effects could have important implications for the demonstration of ignition on the NIF. A two-pronged research effort to clarify the potential importance of these phenomena is proposed. First, the LSP hybrid PIC simulation tool shows promise as a valuable platform for advancing beyond standard fluidbased approaches that underlie current modeling methods. The capability of LSP to run in a multifluid mode or in a kinetic mode provides the opportunity to test and quantitatively assess nonfluid phenomena. Second, the exploding pusher implosion mode provides a comparatively simple platform for isolating potential kinetic effects devoid of the complicating effects of mix and drive asymmetry. As the main shock reflects from the capsule center, locally high ion temperatures are achieved that initiate one or more nuclear reactions of interest that can be well-diagnosed experimentally. The reaction time, "bang time" and yield for each nuclear process can be assessed with current diagnostic techniques and compared with LSP (which includes several principal thermonuclear reaction rates). By comparing LSP simulations in single- and multi-fluid modes the effect of species separation and kinetic phenomena can be isolated and compared with the data. 


\section{Nuclear}

The nuclear fusion of deuterium and tritium provides the main energy-production mechanism in ICF experiments. At adequate densities, the 3.6-MeV $\alpha$ particles released during this fusion process heats the surrounding DT fuel, thereby enhancing the local DT fusion rate. This positive feedback causes the runaway burn that is central to the success of future ICF experiments at the NIF.

Nuclear physics also plays a central role in diagnosing ICF performance. These diagnostics constrain physics models used in ICF simulations, which in turn strengthens the understanding of complex physical processes that occur in HED environments. The nuclear reactions pertinent to diagnostics range from light-ion reactions that give prompt $\gamma$-ray lines and affect the neutron spectrum, to charge-particle reactions and neutron reactions on unstable nuclei. The former is important to prompt diagnostics, such as nTOF and gamma-ray spectrometers, while the latter is important to radiochemical activation analysis. Reactions that affect the neutron spectrum, such as elastic neutron collisions, impact the interpretation of areal densities from nTOF results. Charge-particle reactions probe the amount of mix and therefore provide insights into hydrodynamic instabilities. There are gaps in understanding in many of these nuclear processes, both empirical and theoretical, which must be addressed to ensure that ICF diagnostics reach their full potential and provide accurate descriptions of ICF environments.

With the recent increases in high-performance computing (HPC) resources, great strides have been made in theoretically understanding the spectrum and reactions of nuclei and the underlying origins of the nuclear force. Ab initio calculations, like the no-core shell model (NCSM) and Green's function Monte Carlo (GFMC), of the spectrum s- and p-shell nuclei are routinely performed using realistic two- and three-body nuclear forces. For heavier nuclei, DFT has made significant progress in understanding neutron-rich nuclei, and in particular, the region near the neutron-drip line. The exact lineage of the nuclear force is now being determined from stochastic calculations of quantum chromo- dynamics (QCD), the underlying theory of the nuclear strong interaction that governs the behavior of quarks and gluons.

Current nuclear surrogate experiments are now providing information about particular nuclear reaction cross sections on unstable nuclei, such as neutron capture on Yttrium-88. Planned accelerators of radioactive nuclear isotopes, such as the facility for rare isotope beams (FRIB), will further our understanding of reactions on unstable nuclei, ushering in a regime of 'inverse kinematics' where large unstable nuclei become projectiles on stable targets.

During the course of the NIC, expertise in the area of nuclear diagnostic measurements and the interpretation thereof has increased significantly. The teams in charge of the various diagnostics have overcome many practical issues associated with making measurements in such a challenging environment while simultaneously developing simple analytical and numerical models tying the measurements to more detailed calculations and ultimately to the physical quantities of interest. A recent example of this is the successful fielding of solid radiochemistry measurements [radchem] in indirect-drive experiments. NIF scientists have demonstrated the ability of the capsule neutrons to activate the Au-197 in the hohlraum wall as expected, collect the resulting unstable isotopes Au-196 and Au-198, and measure their activities as an independent indicator of the downscattered neutron spectrum and, hence, the capsule $\mathrm{QR}$. A second example involves a reassessment of the conventional wisdom of which nuclear interactions play a significant role in determining the details of the neutron spectrum escaping an ICF capsule to be measured. The original analysis of the early magnetic recoil spectrometer (MRS) measurements showed a significant deviation from the expected calculated spectrum that was comprised of DT and TT fusion sources and at most a single elastic scatter. Upon observing this difference, a more detailed multiphysics calculation was performed and showed that multiple elastic scatters occur in significant numbers under NIF capsule conditions and that the deuteron breakup reaction $\mathrm{D}(\mathrm{n}, 2 \mathrm{n})$ is a significant source of neutrons in the range of $0-10$ $\mathrm{MeV}$. Both of these results are now routinely 
incorporated in the analysis of both the MRS and the measured neutron images.

\subsection{Priority Research Directions}

\subsubsection{Equations of state for ultra-dense matter and conduction dominated "gas shocks" \\ Introduction}

There are no experimental data constraining the thermodynamic behavior of matter at the very extreme conditions accessed by ignition implosions. With NIF, OMEGA, and Z, physical models for these regimes can now be benchmarked. Three distinct areas that should be addressed as priorities are the compression paths to extreme density of the fuel and the ablator, the release physics of hot and dense matter, and the behavior of ultra-strong shocks in the hotspot. Combined theoretical and experimental efforts will greatly improve the predictive capability for ICF

Compressibility of ultra-dense matter to $2 \mathrm{~kg} / \mathrm{cc}$ : Testing Thomas Fermi Limit.

Determining the compressive equation of state for DT and ablators from the first shock Hugoniot to the extreme peak densities achieved in an ICF implosion is a new scientific frontier. While few calculations and no experiments exist in these extreme density regions, there are hints of new physics emerging for other materials at high pressure, albeit low temperature. For example, Li [15], $\mathrm{Na}[16,17], \mathrm{Al}$ [18], all show a behavior that deviates significantly from Thomas Fermi behavior at extreme densities. While these calculations are at low temperature, they show that at high densities the quantum mechanics becomes increasingly more difficult, not simpler, at least up to gigabar pressures. Moreover, in mixtures at high density, there is an increasing awareness that matter may not behave as an ideal solution. One particular example is the discovery of phase separation at conditions of 1 to many $\mathrm{g} / \mathrm{cm}^{3}$ for $\mathrm{H}-\mathrm{He}$ to near $\mathrm{eV}$ conditions [19]. Finally, recent DFT calculations on $\mathrm{CH}$ mixtures at high density show carbon bonding and fluid behavior hydrogen followed by potential separation of $\mathrm{H}$ and $\mathrm{C}$.
Principal Hugoniot and ramp compression data, combined with DFT and Purgatorio calculations for ablators is the first and easiest step in improving the EOS database. Planar geometry experiments can be carried out to 20 Mbar at OMEGA and $>100$ Mbar at NIF. Data at gigabar pressures can be obtained at high temperature using single convergent shocks and ignition-like implosions diagnosed with ultrahighresolution radiography and Thomson scattering.

For hydrogen fuel, the principal Hugoniot should be extended to 10s of Mbar at NIF. One way to access higher densities is to precompress the sample before shock or ramp compression. Convergent experiments to many gigabar should be performed first at OMEGA, then NIF. These experiments will set a new frontier in dense matter physics and may help us understand why so much energy appears to be stored in the compressed fuel. While several DFT and PIMC calculations have been performed, there are wide disagreements between calculations leading to significantly different predictions for ignition implosions. These differences need to be identified and resolved.

Principal Hugoniot data to 100 Mbar or more requires either new EOS standards or nontraditional techniques for measuring such highpressure states. One such technique is the use of symmetric impact experiments on lasers. This technique seems quite feasible given that the precision pulseshaping capability on NIF makes possible launching of flyer plates for near symmetric impact experiments with flyer velocities of more than $100 \mathrm{~km} / \mathrm{s}$. Another technique that will be required for measuring pressure, density and internal energy for the highest density/convergent experiments is high resolution imaging with advanced $\mathrm{x}$-ray optics such as KB microscopes operating from 1 to $\sim 20$ $\mathrm{KeV}$. To measure temperature of the ablator and fuel, Thomson scattering needs to be developed to be used with radiography. This will enable simultaneous measurements of $\mathrm{P}, \rho, \mathrm{T}$ and potentially collision times throughout the densest regions of an implosion.

In the short term, the conditions in the fuel and ablator need to be measured under ignition conditions. In the longer term, it is important to 
understand the path the materials take to achieve these conditions. Although the end-point may be the same, different paths may differ greatly in the efficiency with which they achieve the same final state. Determining where physical effects such as pressure-induced ionization actually occur may help optimize the achievement of the optimal ignition state.

Development of new standards and approaches for high pressure measurements will enable a wide range of fundamental science experiments with applications to astrophysics, planetary science and high energy density physics. Such precision EOS measurements will lay the foundation for a robust research program on materials at previously inaccessible regimes.

Existing EOS models almost exclusively rely on an assumption of homogeneity. The reliability of EOS models for heterogeneous materials under extreme conditions is essentially untested. It is not known if compounds should become disordered alloys under high compression, if ordering and other alloy-like effects will be important at gigabar pressures, or if more exotic behavior like electride structures can persist to ultra-high pressures. At high densities, the overall energy and pressure for the state will be increasingly dominated by the very-high coldcurve contribution, but differences among different states may still be large enough to favor unexpected structural and electronic phase changes. A platform like NIF is ideally suited for investigating fundamental science questions of this type.

\section{Release and expanded states of shocked and multiply shocked materials}

Release states of the ablator material help set the piston velocity and pressure of the fuel. The release of highly compressed DT helps to set the fuel density profile during later stages of the implosion and is important in setting the correct hotspot conditions. Very little release data exist for these materials, and the experiments that have been done largely show that our methodology for calculating release states (such as in shock timing experiments) is not robust. Finally, the release behavior of hot expanded $\mathrm{Au}$ in part determines the collisional absorption efficiency and charge state distribution for the radiating hohlraum wall.
Significant progress in developing new techniques for measuring release states makes it now possible to collect crucial, ignition-relevant data at OMEGA, Z and NIF. Historically, low pressure release data were collected using low density EOS "standards," such as silica aerogel foams, since their density can be tuned over a broad range below $\sim 1 \mathrm{~g} / \mathrm{cm}^{3}$. However, at higher shock release pressures, no standards are calibrated. Thus, either a significant effort is needed to calibrate new standards at higher pressures or new measurement techniques are required. Deuterium was used as a standard to collect $\mathrm{CH}$ release data in recent shock timing experiments. This technique should be systematically used for other ablator materials. One particular technique recently developed at OMEGA measures the acceleration of a thin SiN witness plate. Here, the shock compressed material releases across a vacuum gap to accelerate a thin $\mathrm{SiN}$ plate whose velocity is measured with a VISAR. This technique provides high fidelity release data into the Mbar regime and can be implemented at NIF or Z. Another technique to be pursued at OMEGA EP and Titan is to isochorically heat the sample with protons or $\mathrm{x}$-rays and image the release wave as it moves into the sample bulk [20].

For the highest density release measurements, phase contrast and/or very high-resolution x-ray imaging will likely need to be used. For example, imaging a multiply shocked ablator releasing into hydrogen could determine the thermodynamic evolution of both the ablator and hydrogen during this key part of the implosion. This will require a high magnification x-ray optic. To determine the temperature of release states, Thomson scattering needs to be developed and used at NIF.

Release measurements provide information that may be difficult or impossible to obtain by other means. First, the thermodynamic release path provides rigorous high-pressure yet lowdensity data in a crucial but largely untested region. Second, release measurements in the vicinity of a phase transition can provide insight into phase transition kinetics. Third, release into vacuum and near-vacuum from heterogeneous materials can provide otherwise-inaccessible information on ion-ion equilibration rates under extreme conditions 


\section{Gigabar wave propagation in the hotspot}

The leading shock wave in the gas interior of a DT implosion, its timing, strength, and structure, helps to set the hotspot adiabat and initiates the stagnation process. As the first gas shock reaches the center of the capsule, pressures rise to the Gbar level. The rebounding wave is so hot that the heat and radiation outrun the compression wave. These three components interact with the fuel to set the final hot spot configuration.

No data exists for the evolution of such strong shocks in gases. Data can be collected in planar geometry for moderate pressure gas shocks to gain experience and provide a first test for models. Ultimately, techniques need to be developed to monitor strong gas shocks in convergent geometry. These techniques may include proton imaging, high-resolution phase contrast imaging, and, possibly diffractive imaging of compression, conduction, and radiative waves. Lower-pressure experiments on capsules may help determine if there is anything fundamentally different about the convergent geometry that is not captured in planar geometries. Such verification may enable some separation of the effects of EOS, opacity and conductivity in a way that is not currently possible, given the highly integrated nature of the ICF experiments.

Keyhole experiments with carefully tailored cones may be useful in measuring the gas shock timing and strength in convergent geometry. This might be useful in separating out effects of the thermal conduction, radiative, and compressive waves both in layered and non-DT layered capsules. Combining radiography, magnetically separated proton time of flight diagnostics and wedge range filter data will help constrain both the shell-gas interface, gas shock arrival time and shock strength in convergent geometry.

Ultimately, techniques need to be developed to monitor strong gas shocks in convergent geometry. These techniques may include proton imaging, high-resolution phase contrast imaging, and potential diffractive imaging of compression, conduction, and radiative waves. For initial experiments, advanced keyhole experiments, magnetic proton time of flight combined with aluminum wedge range filters will begin to provide information on the shock dynamics early in the stagnation of the implosion.

The current understanding of the stagnation process is quite limited. It is likely crucial to gain a detailed understanding of the thermodynamics and kinetics of the initial gas shock, rebound shock and reverberation to higher density of the hotspot. In particular, taking apart the detailed dynamics of the radiation, conduction and compression waves of the initial gas "shock" may provide stringent tests on the physical models defining the hot spot of ignition.

\subsubsection{Opacity research for ignition Introduction}

Accurate opacity models are required for predictive ICF simulations, since photons are a major mode of energy transfer at many stages of the implosion. Absorption and emission of radiation is important in the hohlraum, the ablator, and the implosion core, for both LTE and nonLTE conditions. Key steps for improving opacity modeling include first collecting accurate selfconsistent opacity/emissivity benchmark data for ablator and hohlraum materials up to peak ignition drive temperatures. The second priority is the collection of microscopic data to test specific detailed approximations in opacity theory. To enable part of this effort a new suite of x-ray spectroscopy diagnostics should be fielded on NIF. Finally, a focused effort on advanced atomic modeling which allows practical implementation of LTE and non-LTE calculations for ignition simulations.

\section{Accurate benchmark opacity measurements}

Only a few high-quality benchmark opacity measurements exist, and even in LTE they are nonexistent above $\sim 200 \mathrm{eV}$. Benchmark non-LTE emissivity data for $\mathrm{Au}$ and $\mathrm{U}$ at hohlraum conditions exist, but at present are not absolutely calibrated. Both classes of experiments are key for ignition science. 
Experiments on the $\mathrm{Z}$ platform at Sandia have proven the capability to perform benchmark opacity measurements of $\mathrm{Fe}$ at temperatures up to about $150 \mathrm{eV}$, and while good overall agreement was found in the 800-1350 eV spectral region (corresponding to L-shell transitions in ions with L-shell vacancies), in certain regions of the spectrum, statistically significant deviations between models and experiment are present. Experiments at LCLS have for the first time directly measured the ionization potential depression (IPD) of a plasma - solid-density Al at temperatures up to $180 \mathrm{eV}$ - demonstrating that the ubiquitous Stewart-Pyatt model for IPD is inadequate at high densities. This is important because changes in the treatment of IPD can significantly alter the charge state distribution, thus the electron density, hence affecting both the opacity and EOS of the material.

Benchmark experiments at OMEGA, Z, and LCLS should continue, including experiment design, target fabrication and characterization, diagnostic characterization, post-shot simulations, and analysis and interpretation of the resulting data. While experiments should initially focus on directly relevant materials and plasma conditions, they should be extended to more complex atomic systems to more rigorously test the plasma codes. Further experimental effort should be deployed to

\section{Measuring Ionization Potential Depression (IPD)}

When an ion is embedded in a dense plasma, the energy required to liberate an additional electron is altered from that of an isolated ion in free space - a phenomenon known as IPD. At high densities, the large IPDs can significantly alter the predicted charge state distribution, thus the overall electron density, hence affecting both the opacity and EOS of the material. Almost all opacity codes rely on the half-century old Stewart-Pyatt model, which at high densities is very similar to the simple ion-sphere model. This model has been largely untested and unchallenged, owing to the difficulties in making a hot, uniform density sample, and measuring the ionization energy. However, this has been achieved in recent experiments at LCLS, using the X-ray laser to heat an Al sample. By observing the Ka fluorescence while tuning the photon energy of the LCLS, the IPD can be measured. An accurate analysis of the experiment [21] has shown that the Stewart-Pyatt model does not fit the data, and that the IPDs are far greater than those predicted by this model [22].

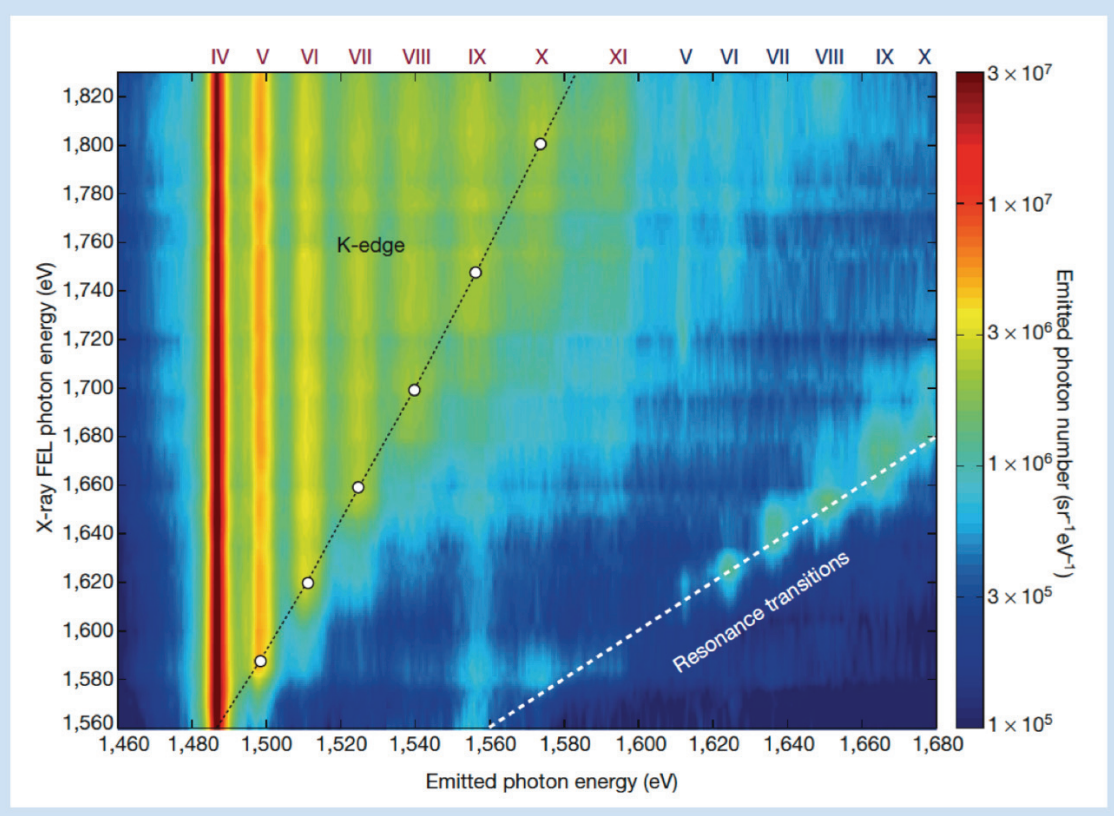

Spectrally resolved $\mathrm{K} \alpha$ emission as a function of $\mathrm{x}$-ray FEL excitation photon energy. The color-coding (bar on right) refers to the emission intensity on a logarithmic scale. Roman numerals (top) indicate the charge state of the emission peak. Open circles: K edges for the various charge states calculated in the SCFLY code according to a modified version of the Stewart-Pyatt model (taken from ref. 21). 
re-evaluate some of the fundamental, and largely untested, first-order assumptions that lie within the complex opacity codes. LCLS has demonstrated its capabilities in generating, via $\mathrm{x}$ ray heating, a uniform density plasma, and with the recent commissioning of the MEC end-station at that facility, which brings nanosecond and femtosecond optical lasers together with the x-ray laser, one might hope that there is further scope for extending these types of experiments, which can be complementary to those that are performed on stand-alone high-power laser platforms. Since physical processes such as the IPD are intimately entwined with other important thermodynamic properties of the plasma - most notably the EOS a key research direction must be the development of experiments in which the pertinent atomic physics and EOS are measured at the same time.

To perform benchmark opacity measurements at plasma conditions found within a NIF hohlraum, it is imperative that the opacity platform being developed on OMEGA be ported to NIF. This requires a spectroscopic capability not currently available on NIF, plus the ability to deal with the classified data produced by such an instrument.

High quality benchmark measurements, especially where codes agree without extant experimental data to test their assumptions, will help constrain simulations and further improve predictive capabilities. Assessing the accuracy of the models according to current theoretical understanding and existing experimental data and assigning explicit error bars would help to quantify uncertainties for target design simulations. Further, this development can help inform choices of materials with greater confidence, increasing design flexibility as the energy transport and loss processes are better understood. Improved understanding will benefit both scientific and programmatic high-energydensity physics research, including astrophysical, stockpile stewardship, and ICF programs.

\section{$X$-ray spectroscopy on the NIF}

While Dante provides a precise measurement of the hohlraum drive, there are significant advantages to be gained by deploying highresolution x-ray spectrometers on the NIF. Such instruments could measure details of the M-band
Au emission; the charge state distribution of the Au ions; the emission of high- $Z$ dopants, both in the ablator and mixed into the fuel; and could potentially provide information about the distribution of the hot electrons caused by LPI. These data will impact our understanding of the deposition of laser energy in the hohlraum, the coupling of the x-ray drive with the ablator, and the physical conditions in the hot spot, complementing information provided by the neutron diagnostics.

A time-integrated high-resolution spectrometer in the central channel of Dante should be installed, using either a variable-spaced grating to cover the soft $(\sim 0.3-2 \mathrm{keV}) \mathrm{x}$-ray waveband and/or a crystal spectrometer to cover the hard $(\sim 2-5 \mathrm{keV}) \mathrm{x}$-ray waveband, combined with an image plate readout. In addition, general high-resolution and time-resolved spectroscopic capabilities should also be fielded on the NIF to enable benchmark experiments on opacity/emissivity, drive and mix.

Measurements of hohlraum wall emission will provide crucial data to test predictions of the hohlraum plasma including hot electrons, plasmagenerated fields, and gradients. Tracers in the hohlraum walls would yield additional drive information and potentially provide a method to tailor the $\mathrm{x}$-ray drive. X-ray spectra from the hotspot can reveal the conditions of a doped-fuel fusion plasma as well as the conditions of any mid-Z materials introduced into the hotspot by mix. In summary, such spectral measurements would provide a new and stringent test of simulation codes, independent of other diagnostics being used to map capsule and hohlraum performance.

\section{Further development of advanced atomic models and opacity codes}

Although it is known how to do detailed, complete atomic models with sophisticated, multiangle transport and self-consistent non-LTE kinetics, there is no tractable way to do this within a multi-dimensional hydrodynamics simulation code. The current state-of-the-art is a Detailed Configuration Accounting (DCA) non-LTE atomic physics model, which, despite it known numerous shortcomings, has resulted in better agreement between simulations and experiments. 
In the near-term the DCA approach should be further developed, including benchmarking against more complete "off-line" opacity simulations. Code comparisons that request postprocessed modeled spectra from a snapshot of relatively simple plasma conditions based on a variety of atomic and transport models might help understand the critical components of what could be termed a "sufficiently accurate" approach to non-LTE modeling and radiation transport. Fundamental theoretical understanding of the properties of atoms within HED regimes must move beyond the average atom/ion in jellium model to realistic multi-center treatments that are not constrained to low temperatures.

Additionally, computational studies aimed at minimizing the uncertainty associated with the choice of radiation transport grouping and algorithms should be explored.

The fact that the EOS integral to the opacity models and the EOS used for modeling hydrodynamic motion are different, leads to some obvious inconsistencies (i.e., different mean ionizations being used within a code for the hydrodynamic response and opacity of an ablating plasma) and also potential inconsistencies not easily recognized in complex rad-hydro simulations.

Effort should be directed toward the development of a hybrid code that utilizes precomputed detailed (e.g., Atomic, Scram, Enrico) non-LTE opacity data tables indexed by electron density, electron temperature, and radiation temperature. While the first generation of such a code would necessarily ignore any non-Planckian aspects of the radiation field, such effects are not dominant, and the code could be extended in subsequent versions to explore these effects.

\subsubsection{Develop an understanding of transport Introduction and kinetic phenomena in ignition}

Several improvements can be made to the physical models that describe how energy is transported and redistributed in space, time, and configuration space, in ICF simulations. In particular, regions where statistical fluctuations do not rigorously permit the hydrodynamic approximation, a methodology is needed that correctly handles the kinetic and particle-based transport of mass and energy. Second in priority is the benchmarking of energy and particle transport models, predominantly for the acceleration and stagnation phases of an implosion. Finally, it is important to produce benchmark data to constrain kinetic models for implosions

Establish a connection between the kinetic and fluid descriptions

ICF design codes make the hydrodynamic approximation, wherein statistical fluctuations and individual particle trajectories are coarse-grained and replaced with species temperatures and guiding-center motion. Though this is certainly advantageous for computational efficiency, key components of the physics are then approximated in a manner that may degrade the predictive fidelity of the simulations. It is therefore crucial for us to study what may be left out in the fluid description, in order to bound any errors which may result from the omission of information pertaining to the lowest length and time scales.

At the most basic level, there is the particle description, in which individual electrons and ions mutually interact with each other and absorb radiation in the process. One of the most important features of plasmas in the fusionburning regime is that the close collisions (electron-electron and between electrons and low$\mathrm{Z}$ ions) are subject to quantum diffraction. In addition, for a plasma in any regime, the more distant collisions are strongly mitigated by screening. This physics of Coulomb scattering is accounted for explicitly in molecular dynamics (MD) simulations of plasmas (though in an approximate way, given that quantum diffraction is handled approximately), while in kinetic and fluid descriptions its effects are convolved into energy exchange rates and transport coefficients which arise from kinetic theory. Since the kinetic theories which are used to derive these rates are themselves only valid at weak plasma coupling and for weak fields, the fluid theories which make heavy use of them are necessarily suspect when addressing plasma conditions which are outside the range where accurate experiments have been performed. Indeed, the ranges of applicability of both the hydrodynamic and kinetic descriptions, 
and the various transport-related quantities of interest, are not precisely known.

Another concern is the level of detail used to describe the statistical distributions of the various particles (and radiation). At the particle (MD) level, all the particles and their different kinetic energies and positions are present, which makes the computations far too demanding to apply directly to, say, an imploding ICF capsule. In the hydrodynamic picture, fluctuations are averaged over, and electrons and ions are each lumped into their own fluids, which at any zone and at a given time are assigned a species temperature. But because the time-scales for plasma heating (shock heating, alpha particle stopping) and cooling (bremsstrahlung, electrical conduction) are comparable with each other and with the disassembly time, the construct of individual species temperatures needs to be carefully examined. In addition, most hydrodynamic codes aimed at studying ICF implosions lump all the ions into a representative "average ion", thereby discounting the very real possibility that different ions may separate in space and in time and behave altogether differently than as an assumed aggregate.

Validating the assumption of a well-defined Maxwellian temperature for a given species or an aggregate of species can be addressed by performing detailed molecular dynamics calculations to investigate the fundamental Coulomb collision processes and compare the results with the existing thermally averaged derivations found in the simulation codes. First, a way should be defined to test this most basic assumption. Kinetic descriptions of the fundamental plasma processes also provide a way to address these issues and investigate the basic transport formulas at a deep level. In particular they can also be used to explore the limits of the simpler transport formulas in the presence of the unique conditions found in the hot spot. Next, comprehensive study should be undertaken of the transport processes in the hot spot using kinetic codes (e.g., LSP).

LSP is a hybrid PIC simulation tool that can be run in explicit mode to assess the shortest spatial and time scales of an imploding plasma approaching ignition conditions. In current research efforts to understand shock morphology, the smallest spatial scales employed are larger than a Debye length and the simulation time steps much greater than an electron plasma inverse frequency. These computational practices need to be checked with finer resolution and ultimately against molecular dynamics simulations for numerical fidelity. The ability to run LSP as a mainline simulation tool for studying plasma shocks requires extensive vetting against explicit PIC methods and MD. The computational requirements are daunting, but not prohibitive.

Molecular dynamics simulations of an imploding ignition capsule are likely not practical over the near term, so approximate methods that bridge the particle and fluid descriptions are required. Hybrid PIC methods or multi-fluid simulations as embodied in LSP are a leading candidate tool to capture the most relevant physical effects in an igniting plasma. However, the integrity of such a numerical strategy requires extensive benchmarking with explicit PIC and MD methods regarding plasma shock morphology. Once the numerical integrity of such a tool is established, its mainline application to an ignition platform is warranted.

Stopping power, electron-ion equilibration, and conductivity in regimes of relevance to ICF

Hydrodynamic simulations used to design ICF implosion experiments rely heavily on numerous energy and particle transport coefficients derived from kinetic theories thought to be valid chiefly for weak plasma coupling and for weak fields and gradients. In fusion-burning plasmas, weakcoupling or not, essentially no experimental data for transport properties exist. Various recent particle-based simulation approaches, such as MD, have revealed discrepancies between the standard kinetic theories and the simulations for quantities such as electron-ion temperature equilibration and charged particle stopping power. Though these discrepancies are not always large in magnitude, fusion burn rates depend very sensitively on the ion temperature, so small differences can have substantial effects on predicted yields. Also, the computational/ theoretical studies completed heretofore have chiefly involved plasmas devoid of high-Z dopants; the presence of an appreciable amount of 
higher-Z ions from the ablator admixed into the DT fuel should complicate the situation greatly.

With new facilities becoming available (GSI, OMEGA-60 and -EP, ORION, LCLS) it may be possible to perform accurate stopping power measurements in dense plasmas. Several approaches are under discussion: using an ion accelerator source with a preformed plasma target, using sheath accelerated protons created by a short pulse laser, and using a fusion proton source. Experiments at TU-Darmstadt, GSI, and the U. of Rochester are about to begin. This effort should be extended and adapted to other facilities as the experimental experience develops and the measurement platform(s) are made more robust. Experimental methods to address electron-ion equilibration have improved over the last decade, in particular with the development of x-ray Thomson scattering techniques to infer the timeevolving electron and ion temperatures. New experimental investigations must be developed to take advantage of this unique probing method along with the large laser facilities that have come online (e.g., OMEGA-EP and ORION).

Measurements of conductivity of the compressed fuel at the dense cool conditions of the implosion adiabat are needed to validate the most recent conductivity models. Initial attempts to measure optical conductivity at these conditions have been performed by Rygg and others, but further work is needed.

On the theoretical front, particle-based approaches are being used to test the assumptions of fluid-based theories. However, these classical MD simulations approximate some of the key physics of quantum plasmas: (1) They represent quantum diffraction in an effective classical manner by employing regularized statistical potentials, and (2) They make use of atomic cross sections, which do not correctly incorporate the effects of high density. Thus, it is important that hybrid kinetic theory-MD approaches be developed so that the salient features of HED plasmas arising from the quantum nature of the electrons (diffraction, electron-ion scattering and radiative processes) can be merged with a proper accounting of time-dependent ion-ion correlations crucial to understand for the accurate prediction of fusion reaction rates.
Existing Purgatorio calculations for conductivities can be augmented with Purgatoriobased stopping-power calculations. These can provide tabulated stopping powers as a function of density, temperature, and ion velocity.

New experiments of ion stopping power in heated, dense matter are under way. The tentative conclusions are that the energy dispersion of the projectile beam and the diagnostic capabilities must be improved to reduce the uncertainties to the point where competing theories and models can be distinguished. For electrical and thermal conductivity, recent work has led to a new $a b$ initio conductivity model for pure H (and D, DT), which so far has been shown to produce similar results, when exercised in an ICF simulation, to that of atom-in-jellium models currently in use for ICF design. However, the electronic structure calculations used to produce this model are sufficiently demanding as to make a direct study of mixtures much less tractable. Ongoing work must involve algorithmic development to speed up such calculations, and the construction of wellvalidated mixing rules aimed at producing conductivity tables for more complex plasmas with some amount of high-Z dopants.

Platforms to measure accurately (in fusionburning conditions) electron-ion equilibration times, electrical and thermal conductivities, and species diffusivity are not available at present. Crucial foundational work must be done to design such focused experiments and to develop the in situ diagnostics capable of making measurements of the required accuracy. Hybrid computational approaches are needed in which classical MD is performed on ions, while the electrons are treated by means of quantum kinetic theory. This, together with the requisite detailed understanding of Coulomb collision physics elucidated by explicit-electron computations, will allow the ps time-scales inherent in the ICF implosion problem to be simulated while retaining the ingredients necessary to fully embody the electron and ion dynamics.

Scientists must ultimately strive for a sufficiently deep understanding of dense plasma energy and particle transport properties such that ICF simulations are not strongly hampered by their uncertainties. It is very likely the case that 
some (possibly multi-) fluid description of the ICF problem will suffice once these transport properties are sufficiently well known. However, obtaining them in a controlled way, from experiments that do not rely heavily on codes and models, which are themselves not well validated, will likely prove extremely challenging. This is why a parallel theory/experiment campaign of basic research is essential. Equally essential is the continued evolution of ICF design sensitivity studies aimed at incorporating the current views of transport uncertainties into the assessment of overall design uncertainties. When moving into uncharted territories of materials conditions (densities, temperatures, etc.) one should be hesitant to expect that paradigms believed to work in the vicinity of ambient conditions will necessarily work here.

\section{Species separation in a strong shock}

Recent LSP simulations of a NIF implosion near "shock flash" show significant D and T separation of the shock front. The implications of this bifurcating shock front on hot spot formation are under investigation. An interesting feature of this simulation is that both the deuterium and tritium components are hotter than the single species case when compared at the same average radius. One interpretation of this result, assuming satisfactory numerical integrity of the simulations, is that the frictional heating generated by the interpenetrating flow of the $\mathrm{D}$ and $\mathrm{T}$ ions within the shock front leads to auxiliary heating or energy dissipation. An important question is whether this effect also exists in the main DT fuel layer during shock transit and to what degree. The separation of $\mathrm{D}$ and $\mathrm{T}$ across a high-Mach number shock during the transit of the gaseous fuel region is also expected to occur, possibly leading to early-time anomalous heating of the hot-spot progenitor gas and a consequent deficit in stagnation pressure. It is important to mention that current shock timing experiments on the NIF use $\mathrm{DD}$ as the surrogate main fuel layer, and no EOS measurements of DT exist. VISAR-based experiments of shock propagation in a DT fuel should be executed to directly test the surrogacy of DD fuels. Such a test would provide muchneeded data on species separation effects and assess whether the adiabat of the fuel is anomalously high compared with fluid-based predictions.

Analysis suggests that a significant amount of resistive heating within the shock front may put the DT fuel on a higher adiabat than predicted by mainline radiation-hydrodynamics simulations, potentially leading to less efficient compressions. The ability of LSP to follow a spherically converging shock over sufficiently long time scales is required in order to test the hypothesis of anomalous shock-driven resistive heating in the DT fuel.

Species separation in thermonuclear fuels is likely to occur, but the degree and impact remain uncertain. Remedial measures are in need of development in the event that species separation is demonstrated experimentally and in simulations. Two candidate workarounds have been proposed: (1) T-rich fuels to approach the single-species limit, and (2) weaker Mach number shocks to minimize the degree of predicted resistive heating.

\section{Develop a tool to evaluate and model kinetic effects}

A hybrid PIC code, such as LSP should be developed into a tool to test the effects of kinetics in ICF experiments and to benchmark hydrodynamic-based simulations. Preliminary analyses and multi-fluid simulations with LSP suggest a potential importance of multispecies/kinetic effects during various stages of fuel assembly in an ICF implosion. Tantalizing exploding-pusher data from the OMEGA laser facility suggest significant differences with fluidbased modeling that may have a kinetic and species separation origin. For example, the measured DD neutron yield compares closely with radiation-hydrodynamics simulation results, but the $\sim 14 \mathrm{MeV}\left(\mathrm{D}^{3} \mathrm{He}\right)$ proton yields are 3-4x larger than modeled. This latter result represents an extraordinary discrepancy that challenges our fluid-based understanding. The impact of nonfluid effects on hot-spot formation and ignition thresholds is not yet known, but an effort to develop such a modeling capability benchmarked against experiments is timely and prudent.

From preliminary work, the "loss of electron tail" phenomenon in the hotspot in first-pass LSP simulations appears to have a minimal effect at 
early times $(<10 \mathrm{ps})$ because of the spherically diverging geometry for long mean-free-path electrons that propagate away from the fuel center. However, further LSP simulations that are conducted for longer time scales, e.g., hundreds of ps, are needed before ruling out this kinetic scenario. The episode following shock flash where electron and ion mean free paths are relatively long and their impact on subsequent hot spot formation uncertain is also amenable to LSP modeling. Such a simulation would assess whether nonlocal transport effects pre-condition the inner layers of the main fuel to significantly alter the mass ablation rates compared with fluidbased predictions. Finally, the late-time effect of Knudsen layer losses of energetic ions from the hot spot on ignition thresholds can be assessed with LSP kinetic modeling, but only over short time scales at present.

LSP simulations in the kinetic mode are being conducted over short time-scales ( $\sim 10 \mathrm{ps})$ to assess the importance of Knudsen layer depletion of energetic fuel ions in the hot spot. These techniques have been adapted to studying loss-ofelectron-tail kinetic phenomena on late-time fuel ablation processes and subsequent hot-spot formation.

A capability to self-consistently model evolving kinetic phenomena in LSP over a longer time-scale ( $>100 \mathrm{ps}$ ) when hydrodynamic profiles can change is needed.

Various kinetic phenomena during hot-spot formation when ion mean-free-paths can be a significant fraction of the hot-spot radius are likely to occur. Whether there importance is sufficient to thwart ignition is not yet established, but developing the capability of LSP to selfconsistently model the integrated effects of kinetic phenomena is needed forthwith.

The need to model shock morphology and propagation in ICF-relevant multi-species plasmas with self-generated fields is urgent. Efforts are ongoing to benchmark LSP's ability to model shock morphology and propagation in a slab geometry, e.g., the Riemann shock-tube problem and the (contact discontinuity) Sod test problem.

The ability to study non-steady shock-front propagation in mixed species plasmas in a converging geometry is a central requirement for adoption of LSP as a mainline multi-species simulation tool for modeling ICF implosions. To this end, the use of pressure and radiation temperature sources in LSP to drive shocks over ICF-relevant time scales is a critical need. Improved EOS and opacity models in LSP are planned. The use of a radiation temperature source in LSP allows a detailed study of ablation front physics in a multi-species plasma, including the role of electric fields on ablation efficiency. Preliminary analysis suggests that the rocket efficiency of a mix-species ablator suffers some degradation, which LSP could validate or refine.

The presumption within the ICF field over the past several decades has been that an averageatom fluid description of the ICF plasma state suffices. However, the growing ICF database suggests that kinetic and multi-species effects may matter at the 1-2 MJ driver scale. The need for a multi-species, kinetic modeling capability to adequately describe the high-energy-density plasma state is becoming increasingly evident. An investment in improving LSP to the point of becoming a mainline ICF modeling tool is justified and timely.

\subsubsection{Nuclear physics for ignition Introduction}

With the gaps in our understanding of key nuclear physics reactions relevant to diagnostics, and motivated by the recent nuclear-related findings at the NIF and other HED facilities, we can identify the three directions for future research:

- Reactions on unstable nuclei using accelerator facilities and reaction theory developments. Our understanding of reactions on unstable nuclei is limited precisely due to the radioactive nature of these nuclei. Surrogate and inverse kinematic experiments at current (LLNL/LBL/Texas A\&M) and future accelerator facilities (e.g., FRIB) provide the best opportunities for measuring cross sections on these nuclei, such as $(n, \gamma)$ and $(n, 2 n)$. Theoretical efforts are also needed in support of these types of experiments. 
- Light-ion reactions from first principles and nuclear diagnostics. Much progress has been made in determining the bound-state properties of light nuclei using first principles nuclear many-body calculations, but limited progress has been made in addressing light-ion reactions, particularly those relevant to ICF diagnostics.

- The interplay between nuclear and plasma physics. ICF conditions will probe new environments where the interplay between nuclear and plasma physics will be inevitable. Our current understanding of this phase space of physics, both theoretical and experimental, is very limited. Research in this area, dubbed plasma nuclear physics, is needed to fully understand ICF environments and shed light on our understanding of conditions observed in early universe evolution and stellar environments.

\section{Reactions on unstable nuclei}

The large neutron brightness obtainable at the NIF, particularly at experiments that achieve ignition, can induce multiple-order neutron reactions on nuclear isotopes far from stability. Radiochemical determination of the amount of production of these isotopes from trace atoms strategically placed within an ICF experiment provides valuable information on the performance of the ICF experiment. For example, proton-rich isotopes, induced through multiple ( $n, 2 n)$ reactions, give information on the neutron hard spectrum since these reactions have thresholds at relatively large energies $(\sim 10 \mathrm{MeV})$. On the other hand, neutron-rich isotopes produced through multiple (n,gamma) reactions give information about the neutron soft-spectrum. Other examples of the types of reactions that occur on trace atoms, and their ICF performance inference, are given in Table 1.
Future nuclear science experiments at the NIF will be integral in nature - the measured quantity of interest will result from a convolution of the underlying nuclear process with some timedependent ICF quantity, such as the neutron fluence. For precise nuclear measurements to be made at the NIF, the profile of the ICF experiment must be well determined. The radiochemical measurements of the nuclear isotopes produced during an ICF experiment is an important process in determining this profile. Table 1 rovides some examples of poorly known reactions of various nuclear isotopes and the accompanying ICF metric that could be constrained if these reactions were known to better precision.

Unfortunately, our understanding of reactions on unstable nuclear isotopes is limited precisely due to their radioactive nature. Surrogate and inverse kinematic experiments at current (LLNL/LBL/Texas A\&M/MSU/Argonne/FSU) and future accelerator facilities (FRIB) provide the best opportunities for measuring reaction cross sections on these nuclei that are directly relevant to ICF experiments, such as $(\mathrm{n}, \gamma)$ and $(\mathrm{n}, 2 \mathrm{n})$. Theoretical efforts are needed to support and supplement these types of experiments. Reaction calculations will have to be utilized to plan and interpret the experiments and to supplement measured cross section data. These (primarily statistical Hauser-Feshbach) calculations require improved input, such as optical-model potentials and level densities, as well as additional theory developments, in particular for describing reactions on those unstable isotopes that are produced in high neutron flux environments. Current optical models work reasonably well for neutron and proton-induced reactions on stable nuclei, but need to be extended to unstable species. Also, the optical potentials chargedparticle reactions, such as $(\mathrm{d}, \mathrm{n})$ and $(\mathrm{t}, \mathrm{n})$, are poorly constrained, even for many stable isotopes.

Table 1. Examples of poorly known reactions on nuclear isotopes and their inferred ICF quantity.

\begin{tabular}{lll}
\hline Isotope & Reaction & Inferred performance \\
${ }^{88} \mathrm{Y}$ & $(\mathrm{n}, 2 \mathrm{n})$ & Hard neutron spectrum \\
${ }^{89} \mathrm{Y}$ and ${ }^{90} \mathrm{Y}$ & $($ n,gamma) & Areal density \\
$\mathrm{Au}$ & (n,gamma) @ $14 \mathrm{MeV}$ & Areal density \\
$\mathrm{Kr}$ & Charged-particle & Mixing and $\rho \mathrm{R}$ of fuel \\
\hline
\end{tabular}


The large flux of 14-MeV neutrons at the NIF induces neutron reactions in an energy regime where pre-equilibrium physics becomes more pronounced, emphasizing the need for better models of pre-equilibrium processes.

Furthermore, contributions from direct capture reactions play a role in high-energy neutron reactions and for isotopes with low-level densities, such as weakly bound systems. These contributions need to be determined, in addition to those included in the statistical reaction models mentioned above. The integration of direct and statistical reaction theory is presently underdeveloped and requires extensions and improvements.

The nuclear reactions on tracer atoms mentioned above, when combined with radiochemical analysis, quantify various aspects of an ICF experiment, such as the neutron fluence. This is especially true when multiple types of tracer atoms with different thresholds are strategically placed within the chamber, hohlraum, and pellet. Utilizing these tracers to their full potential requires adequate knowledge of nuclear cross sections on their unstable isotopes, many of which are poorly known today but can be tackled through combined accelerator-based experiments and theory advances.

\section{Light ion reactions from first principles and nuclear diagnostics}

Much progress has been made in determining the bound-state properties of light nuclei using first principles nuclear many-body calculations, but limited progress has been made in addressing light-ion reactions, particularly those relevant to ICF diagnostics. Of particular importance are nuclear reactions that produce prompt $\gamma$-rays, such as neutron capture on deuterium, ${ }^{2} \mathrm{H}(\mathrm{n}, \gamma)^{3} \mathrm{H}$, the gamma-producing analog of d-t fusion, ${ }^{2} \mathrm{H}\left({ }^{3} \mathrm{H}, \mathrm{n} \gamma\right) \alpha$, and proton capture on tritium, $\mathrm{t}(\mathrm{p}$, $\gamma) \alpha$. These reactions produce $\gamma$ lines that should be readily detected above backgrounds using gamma spectroscopy, thereby giving direct information on the fusion yield. Various (n,n' $\gamma$ ) reactions on ablator material and the hohlraum, such as carbon, oxygen, and gold, are also important since they contribute to $\gamma$ background and therefore impact interpretation of ICF performance from gamma spectroscopy. As NIF experiments approach ignition, these reactions for gamma spectroscopy become more important since the utility of current $\mathrm{x}$-ray diagnostics diminishes due to increased $x$-ray backgrounds.

Reactions that affect the neutron spectrum are important to nTOF measurements, as they impact the interpretation of areal densities. Particular reactions include inelastic and elastic neutron scattering off ${ }^{12,13} \mathrm{C},{ }^{16,18} \mathrm{O}$, and ${ }^{7,8} \mathrm{Be}$. The fusion of two tritium nuclei also produces neutrons that can affect the neutron spectrum.

Two neutron/light ion reactions are in use as $\gamma$-ray diagnostic tools in plastic ablator DT capsule experiments currently under way on NIF: ${ }^{12} \mathrm{C}\left(\mathrm{n}, \mathrm{n}\right.$ ') $[4.4 \mathrm{MeV}]$ and ${ }^{3} \mathrm{H}\left({ }^{2} \mathrm{H}, \mathrm{n} \gamma\right)[16.7 \mathrm{MeV}+$ broad resonance from 9 to $16 \mathrm{MeV}$ ]. The first of these is an interaction with material that nominally only occurs in the ablator material, while the second is a reaction of material present only in significant quantities in the capsule fuel. This segregation of the target isotopes gives independent measures of the conditions within the ablator and the fuel as well as hints at the coupling of the two through mix.

In plastic ablator capsules like those currently being fielded at NIF, the $4.4-\mathrm{MeV}{ }^{12} \mathrm{C}\left(\mathrm{n}, \mathrm{n}^{\prime}\right) \gamma$-ray gives a direct measure of the interaction of fusion neutrons and the ablator material. This interaction can happen in one of two places. The first is in the ablator remaining mass. In this case, fusion neutrons are born in the DT fuel and then escape the capsule through the fuel and ablator having up to a few collisions along the way. In this scenario, ${ }^{12} \mathrm{C}(\mathrm{n}, \mathrm{n}$ ') $\gamma$-rays measured at a long distance from the target give a capsule averaged ablator $\mathrm{OR}$. The second place these collisions can occur is in the fuel itself if ablator material has mixed into the center of the capsule. If the ablator $\mathrm{QR}$ could be measured independently, then the enhancement of the 4.4-MeV $\gamma$-ray signal over that which would have been produced by the in tact ablator represented by the $\mathrm{QR}$ measurement could be used to quantify the amount of ablator mass that has mixed into the DT fuel. Work is well under way to establish this $\gamma$-ray signal as a mix diagnostic. Key uncertainties in this effort are (i) the detailed distribution of the mixed material and (ii) the cross section itself. Inelastic scattering cross 
sections on light ions are not well quantified, and more work still need to be done in this area.

The DT gamma ray is a direct measure of the total number of DT fusion reactions. Because ICF capsules are optically thin to $\gamma$-rays, this measurement is not subject to the uncertainties in the number of collisions through the fuel, ablator, hohlraum, and intervening chamber material.

Beyond experimental and instrumental uncertainties, the largest unknown in this process is the branching ratio between the two DT fusion channels ${ }^{3} \mathrm{H}\left({ }^{2} \mathrm{H}, \mathrm{n}\right)$ and ${ }^{3} \mathrm{H}\left({ }^{2} \mathrm{H}, \mathrm{n} \gamma\right)$. This has recently been measured to a much better accuracy giving a value of $(4.2 \pm 2.0) \times 10^{-5}$ with the former being the dominant reaction [1].

In addition to these diagnostic $\gamma$-rays, several light ion reactions generate what amounts to a $\gamma$ ray background. These reactions include ${ }^{1} \mathrm{H}(\mathrm{n}, \gamma)$, ${ }^{2} \mathrm{H}(\mathrm{n}, \gamma),{ }^{28} \mathrm{Si}\left(\mathrm{n}, \mathrm{n}{ }^{\prime}\right),{ }^{28} \mathrm{Si}(\mathrm{n}, \gamma),{ }^{16} \mathrm{O}(\mathrm{n}, \mathrm{n}$ '), and ${ }^{3} \mathrm{H}(\mathrm{p}, \gamma)$. Each of these reactions is included in production target design calculations, but the level of uncertainty in the cross sections as well as the resulting $\gamma$-ray energy and angle spectra are not always well quantified. Efforts are underway to identify shortcomings in the data and the current understanding of these reactions [23].

Current light-ion evaluation techniques, such as R-matrix and hybrid R-matrix/ab initio techniques, will be augmented with photonchannels to allow studies of light-ion reactions that include prompt gamma rays. Increased access to HPC resources should allow for a systematic study of neutron inelastic cross sections off light- to intermediate-sized nuclei. Nuclear database formats are currently being improved to accommodate these new light-ion evaluations, and the uncertainties and covariances that accompany these evaluations. A central database for these reactions should also be made available to the NIF community to avoid inconsistent use of cross section data, such as outdated (legacy) data and those constrained by poor experiments.

The reactions mentioned above are poorly known, both empirically and theoretically. The recent advances in high-performance computing, however, suggest that a deeper understanding of these reactions can be theoretically obtained within a reasonable timeframe. Continued theoretical development is also needed. Ab initio studies of light ion reactions, such as the resonating group no-core shell model (RG/NCSM), provide the tools and capabilities for performing these studies, while continued studies in lattice QCD and lattice EFT will help constrain the nuclear interactions that serve as input to these studies.

Many of the reactions mentioned above can also be improved upon by new measurements at extant experimental facilities. A dedicated program to perform such measurements should be put in place, with an eye towards reactions in the 14-MeV range. Example facilities are those located at the TUNL and the Ohio State University. The possibility of fielding a monoenergetic neutron source at LLNL should also be considered. Such a facility would allow timely experiments to be performed in support of the NIF.

Measuring ICF performance is essential to understanding and constraining the key physics issues at play at the NIF, both now and in the future. Gamma ray spectroscopy and nuclear time-of-flight diagnostics play a large part in providing this capability, but to adequately infer performance and constrain underlying physics from these diagnostics, particularly those aspects that relate to fusion yield and areal densities, a much stronger theoretical understanding of the nuclear reactions mentioned above is needed. A better understanding of these reactions, when incorporated in ICF simulation codes, will provide confidence in inferred ICF performance and constraints that come about from direct simulation/experimental comparisons.

\section{The interplay between nuclear and plasma physics}

ICF conditions will probe new environments where the interplay between nuclear and plasma physics will be inevitable. The current understanding of this phase space of physics, both theoretical and experimental, is very limited.

Research in this area, dubbed plasma nuclear physics, is needed to fully understand ICF environments.

The dynamic evolution of plasma conditions that could be achieved at the NIF motivates the need to study the complex interplay between 
plasma and nuclear physics. In such conditions, nuclear excited states within heavy nuclei can be populated by atomic processes, such as electron transition due to internal conversion (NEET), or through capture of a continuum plasma electron (NEEC). In addition, at sufficiently high temperatures, the background radiation field can couple directly to the nucleus and induce nuclear excitations and transitions. Because these processes are strongly interacting and many-body in nature, the theoretical (and empirical) understanding of these processes is poor.

Plasma conditions in the warm dense region are also of strong interest to nuclear physics, as electron-screening effects, for example, can potentially alter fusion rates of key light-ion reactions. At sufficient densities achievable at ignition, the bath of degenerate electrons can potentially act as an inhibitor to fusion. Again, our theoretical understanding of screening effects in these environments is limited. Headway into these issues can only be made with a concerted research effort involving both plasma, atomic and nuclear physicists.

Nuclear databases are being modified and adapted to include the effects of the plasma medium. Nuclear experiments are being planned that will verify level density assumptions which have direct bearing on plasma-induced nuclear reactions. Transport codes are being modified to incorporate medium-dependent nuclear cross sections. Simulations from these codes will help prioritize and determine levels of sensitivity of various plasma/nuclear couplings.

The NIF experiments that achieve ignition will usher in a new physics regime. The research efforts mentioned above will be key in understanding and interpreting processes in such HED environments, and guide future HED experiments. Further, this research in Plasma Nuclear Physics will shed light on our understanding of conditions observed in early universe evolution and stellar environments..

\subsection{Conclusions}

The facility infrastructure is now in place for reaching thermonuclear conditions as predicted with our current understanding of many physical properties integral to ICF simulations. The next step in optimizing such implosions at NIF requires a better understanding HED states over an unprecedented range of conditions. While to date, none of the physical models integral to ignition simulations are benchmarked against data over most of the ignition implosion regime, the same infrastructure being used to pursue ignition can be used to make a generational improvement to all of the physical models that underlie ICF simulations. Over the next 3 to 10 years, a focused and sustained effort to benchmark and improve physical models of HED will make such a generational change in our understanding and predictive capability for simulating HED conditions leading to a burning plasma on NIF.

\subsection{References}

1. C. C. Pickard and R. Needs, J. Phys. Condns. Matter 23, 53201(2011).

2. R. More, et al., Phys. of Fluids, 31, 3059(1988).

3. B. Wilson, V. Sonnad, P. Sterne, and W. Isaacs, Journal of Quantitative Spectroscopy and Radiative Transfer 99, 658-679 (2006).

4. S. Hamel et al., submitted to PRB. H. Robey et al., POP 19,42706(2012).

5. M.A. Barrios, D.G. Hicks, T.R. Boehly, D.E. Fratanduono, J.H. Eggert, P.M. Celliers, G.W. Collins, and D.D. Meyerhofer, Physics of Plasmas, 17, 056307 (2010).

6. L. Caillabet et al., PRL, 107, 115004(2011).

7. D. Hoarty et al., HEDP, 8, 50 (2012)

8. J. Eggert et al., PRL, 100, 124503(2008). P. Celliers et al. PRL, 104, 184503(2010).

9. M. D. Rosen et al., HEDP, 3, 180(2011).

10. Y. Lee, R. More, Phys. Fluids, 27 1273(1984).

11. A. Ng, P. Celliers, G. Xu, and A. Forsman, PRE, 52, 4299(1995); P. Celliers, A. Ng, G. $\mathrm{Xu}$, and A. Forsman, Phys. Rev. Lett. 68, 2305 (1992).

12. J.R. Rygg et al., Phys. Plasmas 13, 052702 (2006).

13. K. Molvig et al., PRL accepted July 25, 2012

14. P. Young, M. Foord, A. Maximov, and W. Rozmus, PRL, 77, 1278(1996).

15. I. Tamblyn et al., PRL 101, 75703(2008).

16. T. Matsuoka and K. Shimizu, Nature 458, 186(2009). E. Gregoryanz, et al. Science 320: 1054-1057 (2008). 
17. J. Neeton and N. Ashcroft, Nature 400, 141(1999).

18. C. J. Pickard and R. J. Needs, Nature Materials, 9, 642(2010).

19. J. Fortney and W. Hubbard, ICARUS, 164, 228(2003).

20. T. Bartal et al., Nat. Phys., 8, 139(1012).

21. S.M. Vinko et al, Nature, 482, 59, 2012

22. O. Ciricosta et al, PRL, to be published

23. Y. Kim et al., Phys. Rev. C 85, 061601(R) (2012). 


\subsection{PANEL 6 - INTEGRATED MODELING}

\subsection{Introduction}

This section deals with multi-physics radiation hydrodynamics codes used to design and simulate targets in the ignition campaign. These topics encompass all the physical processes they model and include consideration of any approximations necessary due to finite computer resources. The section focuses on what developments would have the highest impact on reducing uncertainties in modeling most relevant to experimental observations. It considers how the ICF codes should be employed in the ignition campaign. This includes a consideration of how the experiments can be best structured to test the physical models the codes employ.

\subsection{Status of the Physics}

\subsubsection{Underlying processes and properties}

Physical processes modeled in the codes include radiation transport, electron and ion thermal transport, thermonuclear burn and transport of burn products. Production of radiochemical isotopes is also modeled. These codes include models for transport of laser light and the various processes that affect it. They also include a treatment of magnetohydrodyanmics and the effects of magnetic fields on transport processes. Ingredients in the integrated models include atomic physics, in particular, models for opacities and equations of state, both LTE and non-LTE. These codes employ single fluid, twotemperature hydrodynamics, which resolves hydrodynamic instabilities directly.

\subsubsection{Status of theory and modeling}

Simulations used to model experiments in the NIC program fall into two broad categories: 1) integrated hohlraum simulations and 2) capsuleonly simulations. The former type models the hohlraum and capsule and may include patches and reentrant cones in the targets. These can calculate both intrinsic and extrinsic drive asymmetry (due to pointing and power balance errors). Recently, this technique has been extended in HYDRA to include the roughness on the capsule surfaces. The latter capsule-only simulations are often run with high resolution and include roughness on all surfaces, a representative ice groove, the effect of the fill tube and tent and drive asymmetries. The capability to perform these simulations has been developed and validated in the ICF program over many years. The ICF program relies heavily upon simulations carried out in 2D using LASNEX and 2D/3D using HYDRA. Modeling techniques employed in these codes have been tested extensively against experimental data obtained on the Shiva, Nova and OMEGA lasers for a wide variety of experiments. The RAGE code has also undergone experimental validation against ICF experiments to a less extensive degree. Historically, hohlraum simulations performed using the XSN non-LTE opacities and flux-limited conduction have been able to calculate radiation drive temperatures in a wide variety of hohlraums to within $10 \%$ [1]. These account for measured energy scattered out of the hohlraum due to LPI through adjustments to the input laser powers. Simulations of experiments on Nova and OMEGA demonstrated their ability to model pole-to-waist $\left(\mathrm{P}_{2}\right)$ asymmetry as the power balance was varied between the inner and outer cones [2]. The ability of these codes to model the ablative Rayleigh-Taylor instability itself has been validated experimentally in the linear and nonlinear saturated regime, with single mode, two mode, and multimode perturbations, including shape effects in both 2D and 3D [3, 4, 5, 6]. Capsule-only simulations have demonstrated an ability to calculate the variation in yield as the amplitudes of controlled surface perturbations were varied [7].

\subsubsection{Impact of experimental results}

Early vacuum hohlraum experiments on NIF in 2009 showed higher radiation drive temperatures than were predicted with hohlraum temperatures using the historical models.

Simulations using new more detailed models of non-LTE opacities/EOS obtained from the detailed configuration accounting (DCA) model and a detailed model for nonlocal thermal transport produced a higher radiation drive, which was a better match to experimental measurements. With this "high flux model" the plasma temperatures 
and densities calculated for gas-filled hohlraums were closer to values inferred indirectly from LPI measurements, using a linear theory for backscattered power. In contrast to the vacuum hohlraums, the most recent analysis of drive measurements for gas-filled ignition hohlraums indicate this high flux model predicts a flux that is systematically too high, by about $\sim 13 \%$ during the peak. We note that in these gas-filled hohlraums, the evolution of the gold bubbles is restricted by the gas fill and is quite different from the bubble dynamics in vacuum hohlraums.

To account for errors in the energy flow in hohlraum simulations a time varying multiplier is applied to the laser power, constrained to match shock-timing data from keyhole experiments and the bang time. With this adjustment to the "push" delivered, the capsule simulations can match many observables from implosions experiments, such as mass averaged velocity and radius vs. time, shell thickness and peak shell density vs. time. The implosion velocity versus remaining mass fraction agrees within experimental error bars. The shell areal density deduced from the neutron downscatter fraction can also match well the experimental values and is $\sim 85 \%$ the value specified for the point design. And, the ion temperature deduced from neutronic measurements is usually close to the simulated value. The experimental neutron yields are low by a factor of several to 10 times compared to $2 \mathrm{D}$ calculations using the renormalized drive. The hotspot pressure and density inferred from the experiments performed with cryogenic ignition capsules are also low. The calculations show a significant amount of bootstrapping from alpha particle deposition not observed in the experiments due to depressed yields. When bootstrapping due to alpha deposition is disabled in the simulations, the experimental yields are lower than calculated values by a factor of $2-5$ times.

Since energy transfer between crossed laser beams is important on NIF, as was anticipated, a semi-empirical model for the energy transfer is now routinely used in modeling. It is employed by post-processing results of a hohlraum simulation, accounting for the color separation specified between individual beam cones. The model for cross beam transfer contains a single adjustable saturation parameter, which is tuned to match the implosion symmetry. Making use of this model, the simulations match the variation in pole-towaist asymmetry as the color separation between beam cones is varied across a similar set of experiments. Benefitting from guidance provided by simulations, the hotspot symmetry that meets ignition specifications has been achieved by adjusting hohlraum geometry and power balance and wavelength shifts between inner and outer cones.

\subsection{Opportunities for Progress}

In the discussion of specific Priority Research Directions, it is shown in detail how simulations and experiments in each area can advance the ability to perform predictive simulations of NIF experiments and general understanding of the physics involved. Here, a brief overview of the opportunities for progress in these areas and a few others is provided.

Integrated modeling and simulation has a vital role to play in the design, execution, and analysis of NIF experiments. Given the vital role of integrated modeling and simulation in achieving ignition, it is exceedingly important to track down sources of error in the simulations, so the codes can be more effective in guiding the experiments and helping to identify possible missing physics. Simulating the results of experiments has many sources of error. As well as the codes themselves which combine many approximations, there are also the databases containing tables of equations of state, atomic and nuclear physics, and input files that describe the initial "as shot" target geometry, zonal and frequency resolution, laser pulse specifications and instructions to the code describing which models should be used and how. It is important to make progress in each of these areas to better understand and resolve the discrepancies between simulation and experiment. Integrated experiments with incomplete diagnostics are particularly difficult to use as a means to discover which particular models, databases or input errors are responsible for a discrepancy. It is likely that there is more than one thing wrong, and it should be expected that fixing some errors will move the simulated results farther from experiment. Only by doing less integrated experiments will it be possible to validate models 
and simulations of the individual processes involved.

For these reasons, execution of a rigorous, science-based validation campaign provides the greatest opportunity for progress in integrated modeling. This campaign has several components, including a hierarchical directed validation experiment strategy that breaks down the fully integrated experiments into separate parts that can better validate the simulation ingredients, and a hierarchical simulation strategy in which capstone simulations having the highest possible fidelity are done, as well as many modest fidelity simulations. Another component is enhancing the techniques for sensitivity analysis developed within NIC by combining them with quantifications of uncertainties in both inputs and measurements. This can be used to discriminate which inputs and model ingredients are most likely responsible for uncertainties in modeling and to help guide efforts to improve model ingredients. Unraveling the sources of the discrepancies between the predictions of the simulations and the experimental data in non-linear, tightly coupled, fully integrated experiments conducted in the NIC is nearly impossible. Several hypotheses can be put forward for each discrepancy. Directed validation experiments that single out one key physical process in the physical regime encountered in the NIF experiments are therefore crucially needed to cut this Gordian knot. The campaign also includes continued code-to-code comparisons for verification and validation.

Successfully modeling hohlraum energetics is crucially important. Presently, the location of some $170 \mathrm{~kJ}$ of energy $(\sim 10 \%)$ in the NIF ignition hohlraum is not well understood. This involves accurately modeling a plethora of challenging physics models and processes, including equations of state, non-LTE opacities, laser-plasma interactions, non-local electron thermal transport, fluid instabilities, and spontaneously generated magnetic fields.

Recently, several physical processes have been identified that could affect thermonuclear reaction rates in the hotspot. It is important to investigate these processes through both simulations and experiments because the neutron yield in the NIF experiments is a factor $\sim 5$ lower than simulations predict, in spite of the expected areal densities and reasonable ion temperatures being obtained. Physical processes that should be investigated include the separation of deuterium and tritium nuclei by electric fields and barodiffusion, depletion of the tail of the velocity distribution by various loss mechanisms, and non-local electron thermal transport that may play a role in the early formation of the hotspot and the flow of energy into the dense fuel.

In addition to these three Priority Research Directions, the ability to model non-local electron energy transport, a fundamental physical process that plays a key role in hohlraum energetics and may play an important role in the creation and evolution of the hotspot, should be improved. There should also be exploration using both simulations and experiments of the variety of effects spontaneously generated magnetic fields could have on energy transport, fluid velocities, and instabilities, as well as the possible dynamical importance of magnetic fields at and behind the shock fronts that compress the capsule.

\subsection{Priority Research Directions}

\subsubsection{A Science-Based Validation Campaign}

\section{Introduction}

Integrated modeling and simulation has a vital role to play in the design, execution, and analysis of NIF experiments, including those directed toward achieving ignition. For modeling and simulation to play this role, the simulation codes must faithfully predict complex, multi-scale, multi-physics experiments to within the accuracy of diagnostic measurements.

The pathway to predictive capability is a science-based validation campaign in which comparisons of simulation results to experimental data uncover limitations or weaknesses in the integrated model, which is followed by new experiments designed to illuminate further the origins of the discrepancies $[8,9]$. Improvement of models through validation is a continuous process leading to predictive capability.

Decades of validation experiments and predictive science model improvements for nonignition targets lead to a demonstrated ability to 
model well the wide variety of physical effects related to radiation drive and asymmetry in hohlraums and implosion physics, including hydrodynamic instabilities. The capability was developed by testing the models against a wide range of experiments, many of which focused upon specific, individual effects. For NIF ignition experiments, these models must be extended to systems with 100 times as much energy as Nova, much larger spatial and temperature scales, higher convergence ratios and rather different plasma conditions. As these models are extended to these new regimes, comparison with integrated experiments places the greatest stresses on their predictive capability; those simulations require a wide variety of model ingredients, with each having significant bearing on the ability to model the whole system. Isolating specifically the ingredient(s) responsible for disagreements in modeling the integrated experiments alone is exceedingly difficult.

Near-term improvements and approaches to theory and modeling

The Integrated Modeling Panel consequently believes execution of a rigorous, science-based validation campaign provides the greatest opportunity for rapid progress in integrated modeling. Such a campaign should have several components.

The Science-Based Validation Campaign includes continued code-to-code comparisons and appropriate studies of numerical convergence. Such comparisons are an important, though incomplete, way of checking that (i) the codes have implemented the physics models correctly, (ii) the algorithms used in the codes are as accurate and efficient as possible, and (iii) the infrastructure of the codes is operating correctly. In the ICF program code-to-code comparisons are generally done on simplified problems to reduce to a manageable level the effort required to locate sources of differences. Also some codes lack the full set of physics to do the actual problem. As an example, simulations of a simplified problem containing a fill tube run with dissimilar hydrodynamics methods can test if they converge to a similar result. Excessive dependence upon code-to-code comparisons should be avoided. For one, it would tend to result in different codes implementing the very same models. Also codeto-code comparisons produce information regarding similarity of results obtained, but they do not test which methods and models are best at modeling the actual experiments.

The Science-Based Validation Campaign includes a hierarchical directed validation experiment strategy that focuses on crucial aspects of the fully integrated experiments. This is essential because the fully integrated experiments are too complicated to be able to easily identify the reasons for the discrepancies between the predicted and the experimentally measured values, and because the ability to obtain data on the quantities of greatest interest is usually very limited and may at times be impossible. Close and continuing cooperation between simulators and experimentalists will be essential to the success of such validation experiments, as will be the willingness of project leaders to direct resources to the execution of such experiments.

The Science-Based Validation Campaign also includes a hierarchical simulation strategy that includes a number of simulations performed at the highest possible fidelity. High fidelity includes high spatial resolution, or a high level of detail of physics models employed, as well as detailed 3D simulations. The high fidelity simulations provide valuable information about the importance of the various physics models, enabling informed choices to be made about how to reduce the models to make it possible to do many simulations, while retaining the ability to accurately predict the experimental quantities of greatest interest. Many modest fidelity simulations will be needed to do parameter sensitivity studies and to understand the uncertainties in the predictions made by the simulations. The willingness of project leaders to provide the resources necessary to improve existing physics models, develop new physics models, enable the simulation codes to run at scale on existing and future platforms, and carry out a hierarchical simulation strategy will be essential to its success.

Some of the effort within the Science-Based Validation Campaign should be devoted to performing a sensitivity analysis for the most relevant performance metrics to specific model inputs. The NIC has developed a capability to 
perform sensitivity analysis by executing thousands of 1D and 2D capsule-only simulations with HYDRA [10] in which many parameters are varied to map out a multi-dimensional response function of the "quantities of interest" [8]. As has been noted [8], generating response function surfaces for every input parameter variation is not practical. It would result in an under-fit response function that would not be useful. Rather, one must choose a limited number of free parameters based upon physical intuition so that the number of simulations required to generate the response functions becomes tractable. Such a sensitivity analysis yields important insights on which inputs of the reduced model have the most important impacts on the quantities of interest. It is recommended that the program enhance the usefulness of capsule sensitivity studies by combining them with knowledge of the uncertainties in the individual model inputs to assess systematically the impacts of those uncertainties. This will help to identify the specific inputs for which the uncertainties have the greatest impact on quantities of interest. This analysis, combined with quantitative knowledge of the uncertainties in measured quantities of interest could help the program determine which model inputs and ingredients are most strongly associated with errors in modeling of capsule performance. Such an analysis could help the program to determine which focused experiments should receive the highest priority. Examples of approaches for performing this analysis have been reviewed $[8,9]$. As part of simulated ignition campaigns carried out previously within NIC, estimates for uncertainties in the ingredients of many models have been assembled.

With respect to 2D integrated hohlraum simulations, running many thousands of these is not practical at present. Nevertheless, we believe that a systematic assessment of the sensitivities to uncertainties in each of the physics models is feasible and should be performed. This could be accomplished by varying the appropriate settings for each, one at a time, within a set of hohlraum simulations. This version of a systematic sensitivity analysis, combined with knowledge of the uncertainties in inputs, could help to discriminate which model ingredients are most responsible for overall uncertainties in the calculated hohlraum drive. As the model ingredients are improved, this process can be employed iteratively to help speed convergence. [11]

\section{High impact experiments on HED facilities to} address uncertainties in critical physics models

The ICF program has a long history of validating the ability of its modeling tools to successfully simulate essentially every physical process regarded as important in an ignition target design. During the Nova technical contract and for subsequent experiments at OMEGA laser facility, simulations of the performance of various ablators was validated against the results of experiments that measured hydrodynamic performance and a range of hydrodynamic instability effects in planar $[3,4,5,6]$ and converging geometry $[7,12,13]$. The ability to model hohlraum drive [1] and implosion symmetry [9] were also validated for a variety of targets, as was the ability to control laser plasma instabilities $[14,15,16,17,18]$.

Ignition experiments at the NIF probe plasma densities, temperatures, and pressures that are well beyond the ranges that could be achieved and explored on Nova and at the OMEGA laser facility. Thus, the physics models used in the simulation codes for opacities, equations of state, conductivities, etc., have of necessity been applied well outside the regimes for which they were validated in previous experiments. For example, at radiation drive temperatures of NIF, the carbon $\mathrm{K}$ shell experiences significant ionization not present in previous experiments with lower drives. Due to the exigencies of the NIC, the experiments that have been carried out are complex and highly integrated. As a result, each of the experimental results can be related to multiple hypotheses, making it difficult to identify the reasons for the discrepancies between the predicted values and the experimentally measured values.

A series of directed validation experiments that will gather data on individual physical processes for the physical regimes encountered at NIF should be pursued. This data can then be used to validate the models used in the simulation codes in the appropriate regimes. The following are 
examples of the kinds of experiments that should be done:

- Planar foil experiments could measure the performance of various ablators for $300-\mathrm{eV}$ drives using side-on radiography. These could include position vs. time and the rate of foil burn-through. They could also include experiments with planar foils having preimposed perturbations, diagnosed through faceon radiography with a backlighter (see Figure 6.1). By employing foils of the same composition as the capsule ablators, the experiments could be used to validate directly the ability of the simulations to model ablation front growth for relevant drive conditions.

- Experiments measuring the growth of individual features imposed on a backlit capsule implosion diagnosed with radiography could be used to validate simulations of ablation front hydrodynamic instability growth for spherical geometry in the NIF regime.

- Of all the materials employed in the ignition capsules, the program has the least experience with cryogenic DT fuel. Measuring the release of DT in both the keyhole and planar geometry could help constrain the equation of state of DT in this cold, dense regime.
- In planar geometry, a CH/D2 composite target could release through a vacuum onto a witness plate. Measurement of the pressure at the witness plate vs. time using VISAR would help constrain the physics related to early stages of hotspot formation.

- The strength and timing of the stagnation shock could also be measured using the keyhole geometry. Uncertainties in shock timing measurements could be reduced by performing keyhole experiments using DT ice, rather than liquid D2.

- Experiments that measure the growth of fill tube perturbations using high-resolution radiography could further constrain modeling uncertainties.

An alternative path for validation is to identify an integrated experiment matched by the current model. Then, starting from there, march along various paths towards the point design and see where the model breaks down. The HEP4 experiments performed on Nova are a candidate for this approach, since the neutron yield was modeled well by simulations for a set of experiments in which various controlled perturbations were imposed on the capsule surface [9]. The capsules consisted of a single

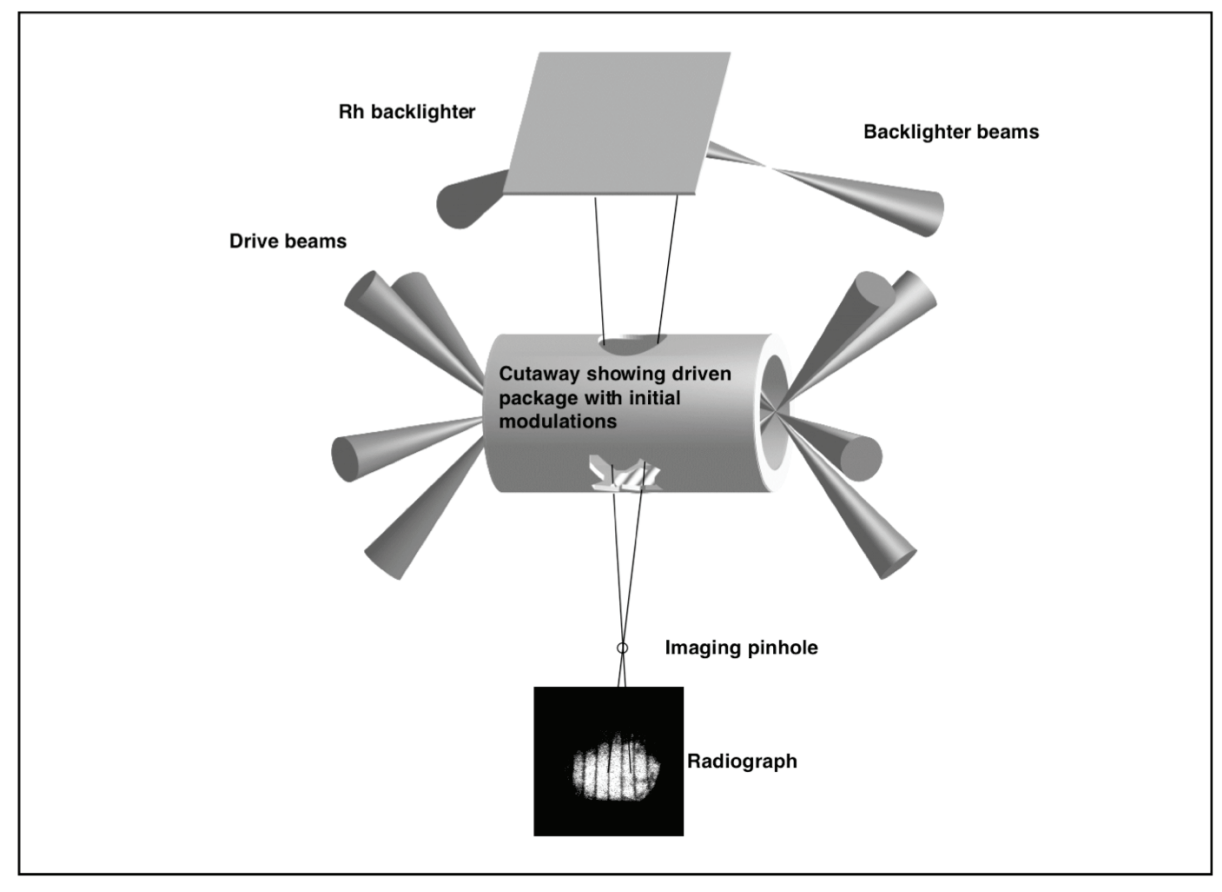

Figure 6.1: Schematic of the geometry for experiments with face-on radiography. 
germanium-doped polystyrene ablator layer of uniform dopant concentration with a thin interior mandrel. They were filled with deuterium gas at room temperature. The specific changes that would be required to march to the point design are the following: (i) going from a two shock to a four shock drive, extending out to $20 \mathrm{~ns}$, (ii) replacing GDP with silicon dopant, (iii) replacing D2 gas with cryogenic DT fuel, (iv) replacing a single dopant ablator layer with a graded dopant layer, (v) raising the peak drive to $300 \mathrm{eV}$, and (vi) obtaining a low fuel adiabat to increase the convergence ratio from $\sim 15$ to $\sim 37$.

\section{Long-term goals and outlook}

Executing a rigorous, science-based validation campaign will enable the ICF program to identify the specific physical effects responsible for the low neutron yields measured experimentally. It will produce a better understanding of where the discrepancy between simulation and experiment becomes significant as one marches away from points in the experimental parameter space where the simulation codes were successfully validated. This will provide a better way of discriminating among competing hypotheses. The end result will be increased ability to do predictive science and greater community and customer confidence in the simulation tools.

\subsubsection{Improved Modeling of Hohlraum Energetics}

\section{Introduction}

Integrated simulations of hohlraums model a wide variety of physical processes, including laser energy deposition, thermal transport and transport of radiation energy. The ablation of various surfaces and the implosion hydrodynamics, including hydrodynamic instabilities, must be resolved. Several factors add to the challenge of hohlraum modeling. The necessity of including detailed inline models for non-LTE kinetics to generate opacities and equations of state is one. In addition, the collisional mean-free-path $\lambda_{c}$ of electrons is long enough to violate the condition for local transport $\lambda_{c}<<L$; there are megagausslevel spontaneously generated B-fields [19] that are of sufficient strength to influence electron transport in the gas-fill, and the B-field topology is complicated. Laser plasma instabilities (LPI), principally stimulated Brillouin (SBS) and Raman (SRS) scattering, redirect significant amounts of energy through backscatter. The SRS electron plasma wave can produce hot electrons, which could preheat the capsule. Cross-beam energy transfer can shift substantial amounts of energy among laser beams. Distorted electron distribution functions due to non-local transport and from intense inverse bremsstrahlung heating can influence plasma profiles. They could also potentially directly affect the production of x-rays from the hohlraum wall through interplay with non-LTE atomic physics. Distorted electron distribution functions might affect the LPI by changing Landau damping rates.

With respect to spontaneously generated magnetic fields, simulations of gas-filled ignition hohlraums carried out using the LASNEX code have shown a Hall parameter $\left(\omega \tau_{c}\right)$ having a peak value of $\sim 5$ in the hohlraum [20], indicating the plasma is not strongly magnetized. And, the magnetic field effects on $\mathrm{x}$-ray drive and associated drive asymmetry are indicated to be rather weak in simulations. So these initial simulations indicate that the principal effect of these magnetic fields on hohlraum performance is a modest change in plasma densities and temperatures in low-density regions interior to the hohlraum, which might influence LPI, and some reduction in mobility of hot electrons.

During the experimental campaigns on Nova and OMEGA, hohlraums were routinely modeled with a high degree of success using XSN average atom opacities and flux-limited electron conduction with a flux limiter of 0.05 . With this long time model, the calculated radiation flux was regularly within $10 \%$ of the experimentally determined value. By comparison, Dante, the principal diagnostic to measure the hohlraum $\mathrm{x}$-ray flux, typically has an uncertainty of $12 \%$ on the flux. As computer power available has increased rapidly, the modeling tools HYDRA and LASNEX have been able to incorporate more detailed and complex models for non-LTE kinetics in the form of the Detailed Configuration Accounting model (DCA). Also, a multi-group model for non-local electron transport has been installed in HYDRA, and later in LASNEX [21], which in principal 
enables a more accurate treatment of the thermal electron heat flow in the hohlraum. Early experiments on the NIF in 2009 using vacuum hohlraums showed higher radiation drive temperatures than predicted with the historic model. Simulations run using the DCA model along with the nonlocal electron transport model, elements of the "high flux model," agreed better with the vacuum hohlraum drive measured on NIF. Plasma temperatures indirectly inferred from LPI backscatter measurements in gas-filled hohlraums on NIF using linear models for LPI backscatter, were also closer to the lower values calculated with the high flux model. In more recent NIF experiments performed with gas-filled hohlraums, the high flux model predicts a drive on the capsule, which is systematically too high by about $13 \%$ during the peak of the pulse.

Recent analysis of time-integrated images of the laser entrance hole aperture imply the numerical simulations, which employ the high flux model, are predicting that it closes somewhat faster than the experiment. This results in an area that is inferred to be $\sim 20 \%$ smaller than the experiment at late time. When this difference in aperture area is accounted for, the drive deduced from Dante is within $4 \%$ of the drive required to match the shock timing data. This is within the experimental error bars on Dante.

To match the shock timing data obtained from keyhole experiments and the bang time, a time varying multiplier on the laser power is applied to hohlraum simulations performed with the high flux model. We note that a series of very recent simulations of shock timing data from keyhole experiments, which use different physics models, is matching well the data for a range of ignition hohlraum experiments without any multipliers on the laser power at all. This module employs the older XSN non-LTE opacities and EOS, new LTE tabular opacities and a higher flux limiter, representative of results obtained with the nonlocal electron transport model. The shots matched include both gold and uranium hohlraums and different rates of rise for the laser pulse [22]. Clearly uncertainties in modeling of non-LTE kinetics and the equations of state can account for significant variations in the simulation results and should be the subject of further research. Other significant uncertainties include nonlocal electron transport, LPI and cross beam transfer and effects of self-generated magnetic fields.

\section{Near-term improvements and approaches to theory and modeling}

First principles modeling of the SRS and SBS backscatter is a grand challenge problem carried out at resolution comparable to the wavelength of light and over the light transit time. Because of the very short spatial and temporal scales involved, it is far too expensive to include inline in a radiation hydrodynamics simulation of a hohlraum. In practice, the effect of laser plasma instabilities has generally been accounted for in hohlraum simulations by subtracting off the backscattered light energy from the laser source. And the effect of cross beam energy transfer has been calculated by post-processing results of a hohlraum simulation. The energy at the laser lens is then modified to model the resulting effect of cross beam transfer. Recently, a model for energy transfer between cross laser beams [23] has been implemented inline in HYDRA. Also semiempirical models for energy backscatter due to SRS and SBS were implemented in HYDRA and LASNEX. A simulation run using these new models would improve modeling by producing a much more self-consistent treatment of energy flow due to LPI effects. For example, the changes in laser momentum deposition due to the cross beam transfer are included, as well as the absorption of redirected energy flow (see Figure 6.2). The resulting plasma conditions can thus be calculated self-consistently. These models should be employed and tested on simulations of experiments, including ignition targets.

Improving modeling of non-LTE kinetics is one of the highest leverage items for improving simulations on hohlraum energetics. There are several areas in the DCA non-LTE kinetics model that should be pursued. One such area lies in the transition from tabulated LTE data to non-LTE calculations. This should occur as soon as nonLTE effects begin to become significant. However, DCA results do not match the LTE data well for the relevant conditions in the hohlraum wall, so the transition region is not well modeled by either LTE or non-LTE methods, and simulation results end up being sensitive to this transition. Also, detection of when the transition 
should occur is not currently informed by kinetics, although this is under development.

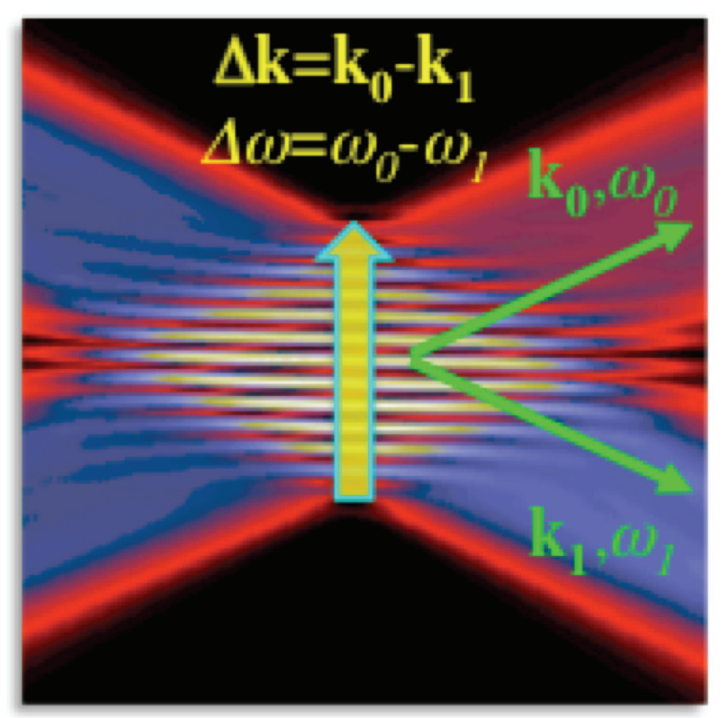

Figure 6.2: Power is transferred from laser beam 0 to beam 1 when the beat wave's phase velocity $\mathrm{v}_{\phi}=\Delta \omega / \Delta \mathrm{k}$ is near the plasma acoustic speed $\mathrm{C}_{\mathrm{s}}$. The process is tunable via $\Delta \omega$, the frequency difference between the two laser beams.

The limitations of DCA in this area are primarily due to the use of very simple atomic models that do not adequately characterize the complexities of ions with 40-50 electrons. A second area requiring improvement is modeling $\mathrm{M}$-band radiation. Here, DCA does a surprisingly good job, as measured by comparisons with benchmark calculations by detailed codes. However, that still allows uncertainties in the range of $20-40 \%$. The limitation here is likely due to an overly simplistic description of excited states of M-shell ions. These are approximated as having a single energy based upon the principal quantum number. Resolving the energy distributions of the levels would improve the overall model accuracy. Lastly an examination of the accuracy of the model for continuum lowering in DCA is warranted.

Atomic models with better fidelity are available from detailed non-LTE codes, but with a level of description that makes their usage several orders of magnitude too expensive for integrated simulations. The improvements necessary for using high-fidelity data inline lie in producing atomic models that reproduce these features with only moderately increased computational requirements over the models currently used with DCA. This is a challenging area for research.

Hohlraum simulations that employ the Schurtz non-local electron transport model [24] are believed to have the most accurate treatment of thermal transport. Alternatively, the simulations done with the high flux model may use fluxlimited thermal conduction to model thermal transport, with the flux limiter calibrated to produce results similar to those obtained from the non-local model. Kinetic codes for electron transport exist that are well suited for modeling non-local transport and include self-consistent Bfields. IMPACT [25] and IMPACTA [26] are implicit 2D (Eulerian x-y grid), parallelized Vlasov-Fokker-Planck codes using the "diffusive approximation" $\mathrm{f}=\mathrm{f}_{0}+\mathrm{f}_{1}$ and including anisotropic pressure $f=f_{0}+f_{1}+f_{2}$, respectively. They include Bz and full B, respectively. The Schurtz model should be tested more thoroughly against a Vlasov-Fokker-Planck calculation of a simplified system, perhaps using IMPACT / IMPACTA, with plasma conditions relevant to a NIF hohlraum. Besides improving our understanding of the accuracy of the non-local model under the conditions specific to NIF experiments, this effort could result in a betterreduced model, having an improved value of a flux limiter.

\section{High impact experiments on HED facilities to address uncertainties in critical physics models}

Opacities of the gold and uranium used in hohlraums are critically important in determining the amount of energy absorbed by the hohlraum wall and retained by coronal plasma ablated off the wall. Measurements of opacities for these materials can help constrain opacity models used in the simulations. Experiments of the type performed by Foord [27] and Heeter [28] produce detailed opacity data for plasma conditions of interest in hohlraums, including the relevant ionization states. More measurements should be made of the opacities of these materials for NIFrelevant plasma parameters to constrain better the opacities models used. Many of these experiments can be performed on the OMEGA laser and other facilities. 
A time-resolved measurement of the closure of the LEH is important for resolving outstanding questions regarding energy balance. Making this measurement for a set of relevant $\mathrm{x}$-ray energies would be desirable.

Hohlraum experiments using different fill gases and pressures to study laser plasma instabilities would be helpful. Varying the laser power would also allow a study of the variation of LPI with plasma conditions.

Dedicated experiments can help test the accuracy of the non-local electron transport models in a long-scale-length plasma. One approach would be to scale up the Gregori [29] and Froula [30] type JANUS (gas-jet) experiments to conditions more indicative of the NIF hohlraum. These involve higher electron density, higher power, two parallel beams (to mock up beam bundles in cones), and two crossed beams (to mock up LEH area). The experiment would use square aperture beams as are used in NIF.

Another approach would be a direct experimental measurement of the heat flow and electron distribution function. This would be a follow on of the experiment of Hawreliak et al. [31]. The experiment would produce more detailed and direct validation data for non-local and kinetic transport models. In particular, the distortion of the electron distribution function by the non-local transport and intense inverse bremsstrahlung heating and the resultant affect upon the heat flow would be of primary interest. This would be done for a laser spot intensity and size like those of a NIF hohlraum, creating similar plasma conditions, though it would not necessarily need to be done on the NIF laser.

\section{New capabilities (diagnostics, models) needed}

One feature of the high flux model is sharply lower predicted electron temperatures in the

\section{Non-LTE Modeling}

Experimental diagnostics that could help constrain non-LTE modeling choices would be particularly useful given that non-LTE modeling uncertainties account for at least 200 ps of the approximately 300 - to 800 -ps discrepancy between observed bang times and predictions.
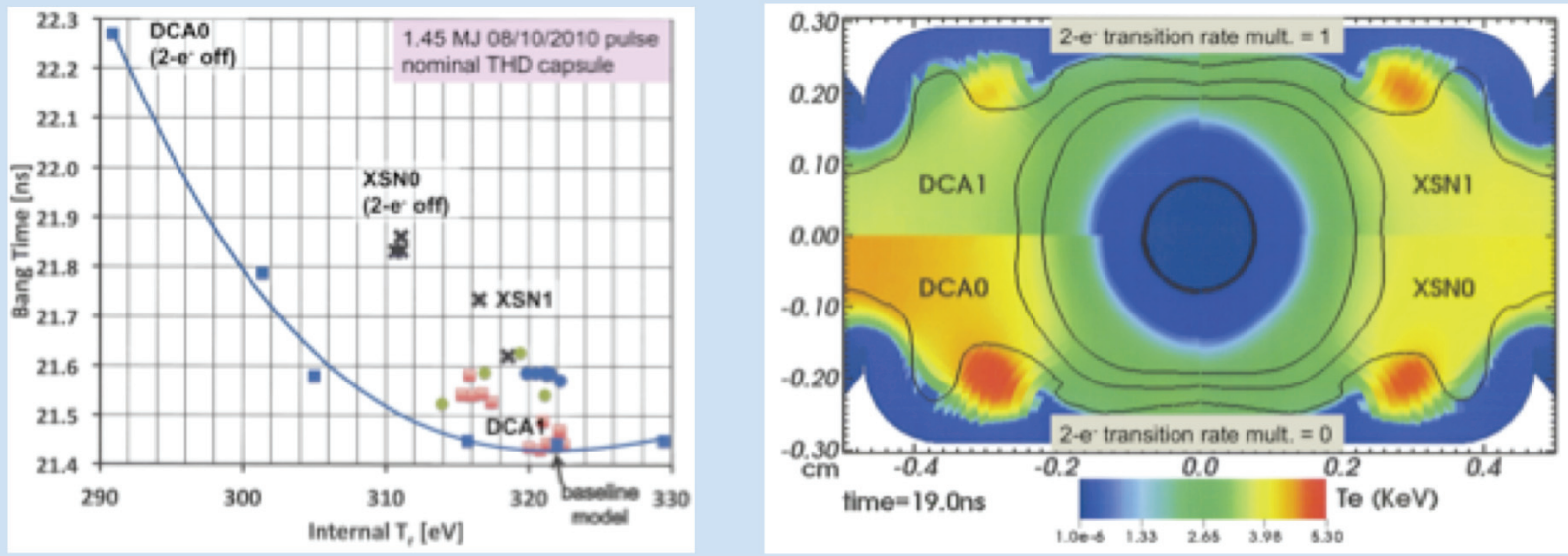

Left: Figure illustrating the sensitivity of the radiation drive and predicted bang times to non-LTE modeling parameter variations. Each point represents the result of a 2D integrated HYDRA hohlraum simulation of a nominal NIF target. Justifiable variations (e.g., dielectronic recombination (DR) rate multiplier of 0.1-2.0) can result in 200 -ps delays in bang times, bringing them closer to the measurements that typically come in over 400 ps later than predicted. The solid line fits through points in which the DR rate multiplier varies from 0.0 to 2.0. Right: Electron temperature maps taken from simulations corresponding to the four labeled points in the figure on the left. The solid lines indicate material boundaries. The top left (DCA1) corresponds to the current baseline model, while the map below (DCA0) shows the effect of turning off DR. The same variations for XSN are shown on the right, with XSN0 representing the old Point Design XSN model. The plasma conditions from the integrated simulations are used to obtain spectra of the backscattered light that can be compared with measurements. (Source: LLNL/M.V. Patel) 
hohlraum. There are presently no diagnostics on NIF that can measure electron densities and temperatures in hohlraum experiments. Electron densities and temperatures in hohlraums have been inferred indirectly, based upon backscatter measurements, using linear models for LPI backscatter. A direct measurement of the plasma electron density and electron temperature is needed to constrain models used in hohlraum simulations. A Thomson scattering diagnostic with a $4 \omega$ probe laser has been proposed for this purpose. In addition, a simultaneous measurement of the magnetic fields in the hohlraum would help the program to understand its role in modifying the plasma temperature profiles. This could be accomplished using proton radiography, measuring along the lines of the sight, similar to the approach taken on the OMEGA laser. With direct simultaneous measurements of the aforementioned plasma quantities, the program would be able to discriminate between the models

Measurements indicate that laser plasma instabilities such as SRS and SBS scatter about $\sim 17 \%$ of the laser energy back out of the hohlraum. This is of the order of the modification in energy input required to match capsule implosion dynamics with the high flux model. Presently, the backscatter is measured on only a handful of NIF beams. Given the importance of backscattered energy on the drive and uncertainties surrounding it, a more comprehensive set of measurements of LPI-induced energy redirection is warranted. LPI-induced energy redirection includes the generation of suprathermal electrons that could be detected by any resulting x-ray emission. To this effect, effort should be directed at improving the spatial and temporal resolution of the FABS (Full Aperture Back Scatter) diagnostic to enhance understanding of SRS and SBS processes and implementing simultaneous measurements on more beams. In addition, it is recommended that the temporal resolution of the Dante and filter fluorescer (FFLEX) diagnostics be improved.

With respect to modeling, improving the Vlasov-Fokker-Planck codes to model r-z and rtheta geometries would allow better applicability to hohlraum and greater overall flexibility. Improving the overall physics capabilities of those codes would make them more useful for assessing the importance of kinetic effects in actual hohlraum experiments.

A better theoretical understanding of the nonlinear saturation mechanism for energy transfer between crossed laser beams is important. This has significant impact on the energy flow in the hohlraum.

An improved, more comprehensive and robust treatment of magnetized transport effects,

particularly Righi-Leduc heat flow $(\mathrm{k} \wedge)$ and Nernst advection $(\mathrm{b} \wedge)$, into hohlraum simulation codes is important. This would enable a better assessment of the significance of magnetic field effects upon transport and resulting modifications to plasma temperature and density profiles. This work is under way in HYDRA and LASNEX at different stages of development in 2D and 3D and extends over a several year time frame.

\section{Long-term goals and outlook}

By validating individual elements of the models used in hohlraum simulations, the program will improve validation of the integrated model itself. The ultimate result of these efforts will be an improved predictive capability for modeling capsule drive and preheat. An improved understanding can ultimately lead to achievement of a higher radiation drive. The figure of merit of an ignition capsule implosion has a very strong dependence upon implosion velocity and thus the peak drive. As a result, achieving a higher radiation will strongly benefit the campaign to achieve ignition.

\subsubsection{Kinetic Effects on Thermonuclear Yield}

\section{Introduction}

Recently, several processes have been identified that could modify thermonuclear reaction rates due to the fuel plasma being a nonideal fluid. These processes include the separation of deuterium and tritium nuclei by electric fields and barodiffusion [32], the loss of reactive ions in the high-energy tail of the distribution function due to their long mean free paths [33], as well as various infinite medium processes such as tail enhancement by large-angle alpha particle 
collisions, tail depletion due to the DT reaction itself and lack of time to fill out the tail. Non-local transport can also play a role in the early formation of the hotspot and the heat flow from the hotspot into the dense fuel. These kinetic and non-local effects are more significant when the first shock reaches the center of the capsule. In a cryogenic NIF capsule, the hotspot mass increases many times during the implosion due to conductive ablation of fuel from the dense capsule shell. The compression of the hotspot, coupled with the increase in its mass, cause the collisionality to increase. For example, when the capsule has converged to minimum radius and the hotspot is approaching ignition, for a central density of 100 $\mathrm{g} / \mathrm{cm}^{3}$ and the ion temperature of $5 \mathrm{keV}$, the hotspot is over 1000 deuteron mean free paths in radius. As a result, most of these effects will be small as the fuel is beginning to ignite and will become more important during vigorous thermonuclear burn. Analysis done to date suggests these effects would not be expected to account for the yields being low by a factor of several in current NIC experiments with ignition capsules. Given that experiments show low yields in spite of the expected areal densities and reasonable ion temperatures being obtained, the Integrated Modeling Panel recommends that the aforementioned effects be assessed systematically using the best available models.

\section{Near-term improvements and approaches to theory and modeling}

In the near term, the best existing kinetic models and codes should be applied to ignition conditions to learn more about when these various processes are important and to estimate their impact. This effort should include using PIC codes such as LSP [34] to investigate simplified localized geometries and to validate the models used in larger-scale codes like Vlasov-FokkerPlanck [35]. Among PIC codes, LSP is well suited because it can run in 1D spherically converging geometry and contains and expanded set of physical models. The Vlasov-Fokker-Planck codes listed enable one to study ion kinetics or electron kinetics as needed. These tools enable the program to study all of the aforementioned processes. Specific effects can be studied using idealized problems that resemble the hotspot and capsule shell geometry of the ignition capsule. In addition, plasma profiles from a 1D simulation of an ignition capsule can be linked near peak implosion velocity for example to LSP and the simulation carried through. By comparing the evolution of the profiles between the radiation hydrodynamic code and the LSP PIC code, the significance of effects such as species separation can be assessed for a specific target design. If any of the specific effects described above appear to be significant, then reduced models of these processes should be developed in the short term and tested against PIC and VFP codes. Once validated, they would be implemented in multi-dimensional hydrodynamic and transport codes. This would enable these effects to be evaluated self-consistently in a full physics code. Efforts have begun to assess these effects using the LSP code [36].

\section{High impact experiments on HED facilities to address uncertainties in critical physics models}

Shock timing experiments performed in the keyhole geometry to date have used DD as a surrogate main fuel layer. In the actual DT cryogenic capsule, effects of rarefactions launched as individual shocks break out is accounted for by scaling data from DD equation of state measurements. This is a source of uncertainty in the shock timing obtained. Plans exist to conduct VISAR experiments of shock propagation in a DT fuel layer to test the surrogacy of the DD fuels. Such tests would provide required data on species separation effects. It would also allow for an assessment of whether the adiabat of the fuel is anomalously high compared with predictions of the radiation hydrodynamics codes.

\section{New capabilities (diagnostics, models) needed}

Assuming that further analysis reinforces the need for better non-ideal fluid models in the hydro codes, we should develop models that can capture the important physics without overburdening our computational resources. Adding electric fields and barodiffusion to multi-dimensional hydrocodes should be reasonably straightforward and inexpensive to run if limited to a single velocity "diffusion drag" model. Developing a non-local or multi-group model to capture the nonMaxwellian ion tail distribution and using it consistently should also be possible if limited to a 
nearly isotropic approximation. In the interests of speed and robustness, we should probably first develop these models in the absence of magnetic fields, but it will be necessary to include magnetic fields in true multi-dimensional simulations.

An experimental measurement of electric fields in the hotspot is possible using proton radiography. This technique has been carried out in direct-drive implosion experiments fielded on the OMEGA laser [37], where strong electric fields were reported to be measured inside the imploding capsule. Proton radiography measurements of electric fields in the hotspot of capsule implosions should be made on NIF. These would yield valuable information to compare with kinetic models of the aforementioned effects.

\section{Long-term goals and outlook}

In the long term, any kinetic model for the hotspot should be validated by experimental measurements. This seems difficult to do given the integrated nature of ignition experiments. The ignition campaign may have to be satisfied with models in hydro codes that can reproduce the effects seen in Fokker-Planck and PIC codes for relevant plasma conditions. By assessing the several aforementioned kinetic effects with the best existing models and codes, whether any are significant contributors to the yield discrepancy can be resolved. Eliminating specific effects allows the program to focus on other hypotheses. To the extent the investigations warrant, this research direction will result in the development of non-ideal fluid models for more accurate modeling of hotspot physics and associated yields.

\subsection{Conclusions}

The integrated modeling panel advocates the following high priority research directions:
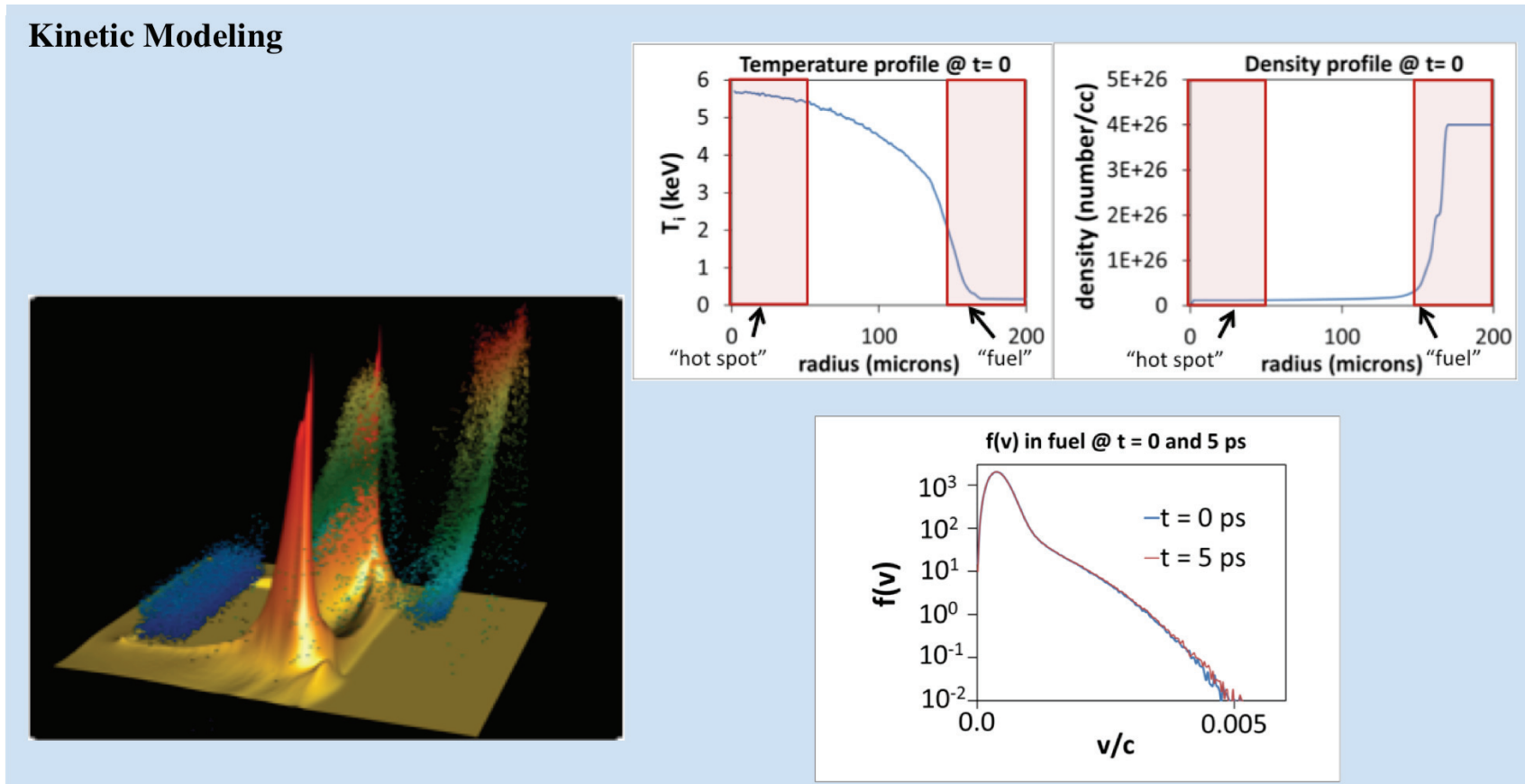

Particle-in-cell (PIC) codes can assess how thermonuclear yield is affected by the fuel plasma being a non-ideal fluid. The PIC simulation resolves plasma particles both in physical and velocity space with self-consistent electric and magnetic fields. Left: Electric field (surface plot) driven by laser light accelerating bunches of particles as they move through and exit a target in a simulation performed with the LSP PIC code. Particle height indicates particle energy. The PIC simulation resolves particles both in physical and velocity space with self-consistent electric and magnetic fields. Right: Top plots show radial electron density and temperature profiles respectively representing conditions in the hot spot of an ignition capsule. Bottom plot shows evolution of deuterium ion velocity distribution function in hot spot at 0 and $40 \mathrm{psec}$ from LSP simulation. Slight depletion of the tail ions results from energetic ions escaping the hot spot. LSP simulation can assess the affect upon fusion reactivity. 


\section{1) Science-Based Validation Campaign}

- Employ hierarchical directed validation experiments that focus on crucial aspects of the fully integrated experiments. These include planar foil experiments to test ablator performance and growth of imposed perturbations. Experiments in both planar and keyhole geometry to examine physics of stagnation for cryogenic DT or DD are also valuable.

- Employ a hierarchical simulation strategy that includes a number of simulations performed at the highest possible fidelity, including high spatial resolution, high detail in the physics models employed and detailed 3D simulations.

- Enhance usefulness of sensitivity analysis studies by combining with quantification of uncertainties in both inputs and measurements. Use this to discriminate which inputs and model ingredients are most responsible for uncertainties in modeling and to help guide efforts to improve model ingredients.

- Continue code-to-code comparisons, focusing on simplified problems.

2) Improved Modeling of Hohlraum Energetics

- Simulate experiments using the new inline models in the hydrocodes for cross beam energy transfer and SRS and SBS backscatter.

- Improve DCA non-LTE kinetics, including accuracy in transition region between LTE and non-LTE. Improve energy resolution of excited states. Assess more accurately the model for continuum lowering.

- Test the non-local electron model against Vlasov-Fokker-Planck code on simplified geometry for plasma conditions relevant to the NIF hohlraum.

- Make more extensive measurements of opacities of hohlraum wall materials under NIF-relevant plasma conditions.

- Perform gas jet experiments to test a non-local electron transport model in long scale length plasmas for NIF-relevant conditions.

- Experimentally measure of heat flow and electron distribution function for NIF-relevant plasma conditions.
- Measure plasma electron temperature and density via Thomson scattering.

Simultaneously measure magnetic fields via proton radiography.

- Deploy a more comprehensive set of measurements of LPI-induced energy redirection. This includes improving the spatial and temporal resolution of the FABS and measuring backscatter on more beams .

- Develop a better theoretical understanding of the non-linear saturation mechanism for energy transfer between crossed laser beams.

- Implement a more comprehensive and robust treatment of magnetic fields effects, including Righi-Leduc heat flow and Nernst advection.

\section{3) Kinetic Effects on Thermonuclear Yield}

- Apply PIC and Vlasov-Fokker-Planck codes to study several kinetic effects and quantify their effect upon the yield (see sidebar below). These processes include the separation of deuterium and tritium nuclei by electric fields and barodiffusion, the loss of reactive ions in the high-energy tail due to several effects. Nonlocal transport in the hotspot and surrounding dense fuel is also of interest.

- If results of kinetic simulations indicate a need, develop a reduced model for the physical effect and implement into hydrocodes for selfconsistent treatment.

- Conduct VISAR experiments of shock propagation in a DT fuel layer in keyhole geometry to test the surrogacy of the DD fuels. This would also provide required data on species separation effects.

- Deploy proton radiography to measure electric fields in the hotspot on NIF. This will yield valuable data for comparison with kinetic models of the aforementioned effects.

\subsection{References}

1. S. Glenzer et al., Phys. Rev. Lett. 802845 (1998)

2. R. Turner et al., Phys. Plasmas, 102429 (2003), UCRL-JC-135811

3. M. Marinak et al., Phys. Rev. Lett. 804426 (1998)

4. B. Remington et al., Phys. Plasmas 2241 (1995) 
5. S. Weber et al., Phys. Plasmas 13652 (1994)

6. B. Remington et al., Phys. Fluids B 4967 (1992)

7. M. Marinak et al., Phys. Plasmas, 32070 (1996)

8. M. L. Adams and D. L. Higdon. Assessing the Reliability of Complex Models: Mathematical and Statistical Foundations of Verification, Validation, and Uncertainty Quantification. The National Academies Press, 2012. ISBN 978052111301.

9. W. L. Oberkampf and C. J. Roy. Verification and Validation in Scientific Computing. Cambridge University Press, 2010. ISBN 9780521113601.

10. POP 19056316 (2012)

11. C. Graziani, T. J. Loredo, and M. Anitescu. Adaptive design of computer experiments and simulation fidelity hierarchies. Technical report, University of Chicago Flash Center for Computational Science, 210. http://flash.uchicago.edu/site/techreports/adapt ive design.pdf

12. P. Amendt et al., Phys. Rev. Lett. 89165001 (2002)

13. S. G. Glendinning et al., Phys. Plasmas 72033 (2000)

14. D. Froula et al., Phys. Plasmas, 14055705 (2007)

15. D. Froula et al., Phys. Rev. Lett. 101115002 (2008)

16. D. Froula et al., Phys. Rev. Lett. 103045006 (2008)

17. D. Froula et al., Phys. Plasmas 17056302 (2010)

18. H. Robey et al., Phys. Plasmas 17056313 (2010)

19. Li et al., Phys. Rev. Lett. 102, (2009)
20. R. Town, Bull. Am. Phys. Society, DPP, 49, 2004, pp. 25

21. G. P. Schurtz, P. D. Nicolai, and M. Busquet, Phys. Plasmas 7, 4238 (2000)

22. Harry Robey, private communication

23. P. Michel et al., Phys. Plasmas 17, 056305 (2010)

24. G. P. Schurtz, P. D. Nicolai, and M. Busquet, Phys. Plasmas 7, 4238 (2000)

25. R.J. Kingham and A.R. Bell, J. Comput. Phys. 194, 1 (2004)

26. A.G.R. Thomas, et al., New J. Phys, 11, 033001 (2009)

27. M. Foord, Phys. Rev. Lett. 85, 992 (2000)

28. R. Heeter,Phys. Rev. Lett. 99, 195001 (2007)

29. G. Gregori,Phys. Rev. Lett. 92, 205006 (2004)

30. D. Froula,_Phys. Rev. Lett. 98, 135001 (2007)

31. J. Hawreliak et al.,J. Phys B 37, 1541 (2004)

32. P. Amendt, S. C. Wilks, C. Bellei, C. K. Li \& R. D. Petrasso, Phys. Plasmas 18, 56308 (2011)

33. K. Molvig, N. M. Hoffman, B. J. Albright \& R. B. Webster, LA-UR 12-0150 (2012)

34. D. R. Welch, D. V. Rose, B. V. Oliver \& R. E. Clark, Nucl. Instrum. Methods Phys. Res. A 464, 134 (2001)

35. O. Larroche, Eur. Phys. J. D 27, 11 (2003), M. Casanova et al., PRL 67, 2143 (1991), and A. G. R. Thomas, M. Tzoufras, A. P. L. Robinson, R. J. Kingham, C. P. Ridgers, M. Sherlock \& A. R. Bell, J. Comp. Phys. 231, 1051 (2012)

36. S.C. Wilks, private communication

37. C. K. Li et al., Phys. Rev. Lett. 100, 225001 (2008) 
APPENDIX A

WORKSHOP ON THE SCIENCE OF FUSION IGNITION ON NIF

AGENDA

May $22-24,2012$

San Ramon Marriott Hotel

San Ramon, CA

Tuesday, May 22

5:00-

$8: 00 \mathrm{pm}$

Evening registration and reception

Wednesday, May 23

\section{Morning Plenary Session}

07:30 am Continental Breakfast

08:00 am Welcome (Penrose "Parney" Albright, LLNL Director)

08:10 am NNSA Welcome (Jeff Quintenz, NNSA ICF Director)

08:20 am NIF Welcome (Ed Moses, LLNL NIF \& Photon Science, Principal Associate

Director)

08:30 am Workshop Goals and Deliverables (William Goldstein, LLNL Physical and Life Sciences, Associate Director)

08:45 am Ignition Overview and Status (John Lindl, NIF Chief Scientist)

09:45 am Ignition Measurements (Joe Kilkenny, NIF Diagnostics Leader)

10:30 am Coffee Break

10:45 am Panel Introductions (10 minutes each)

1. Laser propagation and $\mathrm{X}$-ray generation

2. X-ray transport and ablation physics

3. Implosion hydrodynamics

4. Stagnation properties and burn

5. HED Materials cross-cut

6. Integrated modeling

1:45 pm Hosted Lunch and Group Photo

12:00 pm Afternoon Parallel Panel Breakout Sessions (lunch continues)

03:00 pm Coffee Break

05:00 pm Panel Adjourn

05:30 pm Hosted Dinner

07:00 pm Parallel Panel Breakout Evening Sessions (Optional) 
Thursday, May 24

\begin{tabular}{ll}
\hline & Morning Plenary Session \\
07:30 am & Continental Breakfast \\
08:00 am & Initial Panel Outbriefs (10 minutes each) \\
09:00 am & Parallel Panel Breakout Morning Sessions \\
12:00 pm & Hosted Lunch \\
01:00 pm & Parallel Panel Breakout Afternoon Sessions \\
02:30 & Coffee Break \\
03:00 pm & Final Panel Outbriefs (15 minutes each) \\
& \\
& 1. Laser propagation and X-ray generation \\
& 2. X-ray transport and ablation physics \\
& 4. Implosion hydrodynamics \\
& 5. HED Materials cross-cut \\
& 6. Integrated modeling \\
04:30 pm & Workshop Wrap Up \\
05:00 pm & Workshop Adjourns
\end{tabular}




\section{APPENDIX B：WORKSHOP PARTICIPANTS}

\begin{tabular}{|c|c|c|}
\hline Last Name & First Name & Organization \\
\hline Abarzhi & Snezhana & University of Illinois at Chicago, University of Chicago \\
\hline Afeyan & Bedros & Polymath Research Inc. \\
\hline Al-ayat & Rokaya & Lawrence Livermore National Laboratory \\
\hline Albright & Parney & Lawrence Livermore National Laboratory \\
\hline Albright & Brian & Los Alamos National Laboratory \\
\hline Amendt & Peter & Lawrence Livermore National Laboratory \\
\hline Ampleford & David & Sandia National Laboratories \\
\hline Arnett & Dave & University of Arizona \\
\hline Atzeni & Stefano & Università degli Studi di Roma "La Sapienza" \\
\hline Azechi & Hiroshi & Institute of Laser Engineering, Osaka University \\
\hline Bahukutumbi & Radha & Laboratory for Laser Energetics, University of Rochester \\
\hline Bailey & James & Sandia National Laboratories \\
\hline Baisden & Patricia & Lawrence Livermore National Laboratory \\
\hline Barnes & Cris & Los Alamos National Laboratory \\
\hline Barty & Christopher & Lawrence Livermore National Laboratory \\
\hline Batha & Steven & Los Alamos National Laboratory \\
\hline Betti & Rlccardo & Laboratory for Laser Energetics \\
\hline Bingham & Robert & $\begin{array}{l}\text { Science and Technology Facilities Council / Rutherford } \\
\text { Appleton Laboratory }\end{array}$ \\
\hline Birely & John & John H. Birely Sole Proprietor \\
\hline Boehly & Thomas & Laboratory for Laser Energetics, University of Rochester \\
\hline Callahan & Debra & Lawrence Livermore National Laboratory \\
\hline Casey & Daniel & Massachusetts Institute of Technology \\
\hline Casner & Alexis & Commissariat á l"Energie Atomique \\
\hline Celliers & Peter & Lawrence Livermore National Laboratory \\
\hline Cerjan & Charles & Lawrence Livermore National Laboratory \\
\hline Chittenden & Jeremy & Imperial College \\
\hline Clark & Tim & Los Alamos National Laboratory \\
\hline Clark & Daniel & Lawrence Livermore National Laboratory \\
\hline Clouse & Christopher & Lawrence Livermore National Laboratory \\
\hline Collins & Gilbert & Lawrence Livermore National Laboratory \\
\hline Correll & Donald & Lawrence Livermore National Laboratory \\
\hline Crandall & David & Department of Energy \\
\hline Curry & John & National Institute of Standards and Technology \\
\hline Delettrez & Jacques & University of Rochester \\
\hline Edwards & Johnv & Lawrence Livermore National Laboratory \\
\hline Epstein & Reuben & Laboratory for Laser Energetics, University of Rochester \\
\hline Fernandez & Juan & Los Alamos National Laboratory \\
\hline Fincke & James & Los Alamos National Laboratory \\
\hline
\end{tabular}




\begin{tabular}{|c|c|c|}
\hline Fisch & Nathaniel & Princeton University Plasma Physics Laboratory \\
\hline Freeman & Richard & The Ohio State University \\
\hline Frenje & Lars Johan & Massachusetts Institute of Technology \\
\hline Froula & Dustin & Laboratory for Laser Energetics, University of Rochester \\
\hline Fryer & Christopher & Los Alamos National Laboratory \\
\hline Garbett & Warren & Atomic Weapons Establishment \\
\hline Gauthier & Pascal & Commissariat á l'Energie Atomique \\
\hline Gericke & Dirk & University of Warwick \\
\hline Giuliani & John & Naval Research Laboratory \\
\hline Glenzer & Siegfried & Lawrence Livermore National Laboratory \\
\hline Glimm & James & Stonybrook University \\
\hline Goldstein & William & LLNL/Physical and Life Sciences Directorate \\
\hline Goncharov & Valeri & Laboratory for Laser Energetics, University of Rochester \\
\hline Grim & Gary & Los Alamos National Laboratory \\
\hline Haan & Steven & Lawrence Livermore National Laboratory \\
\hline Hammer & James & Lawrence Livermore National Laboratory \\
\hline Hansen & Stephanie & Sandia National Laboratories \\
\hline Haynes & Donald & Los Alamos National Laboratory \\
\hline Heeter & Robert & Lawrence Livermore National Laboratory \\
\hline Herrmann & Mark & Sandia National Laboratories \\
\hline Herrmann & Hans & Los Alamos National Laboratory \\
\hline Hicks & Damien & Lawrence Livermore National Laboratory \\
\hline Hill & Edward & Imperial College, London \\
\hline Hinkel & Denise & Lawrence Livermore National Laboratory \\
\hline Hockaday & Mary & Los Alamos National Laboratory \\
\hline Hoffman & Nelson & Los Alamos National Laboratory \\
\hline Hohenberger & Matthias & Laboratory for Laser Energetics \\
\hline Holzrichter & John & Lawrence Livermore National Laboratory \\
\hline $\mathrm{Hu}$ & Suxing & Laboratory for Laser Energetics, University of Rochester \\
\hline Hurricane & Omar & Lawrence Livermore National Laboratory \\
\hline Joshi & Chandrashekhar & University of California, Los Angeles \\
\hline Katz & Jonathan & Washington University in St. Louis \\
\hline Kauffman & Robert & Lawrence Livermore National Laboratory \\
\hline Keane & Christopher & Lawrence Livermore National Laboratory \\
\hline Kerman & Arthur & Massachusetts Institute of Technology \\
\hline Key & Michael & Lawrence Livermore National Laboratory \\
\hline Kilkenny & Joseph & General Atomics \\
\hline Kingham & Robert & Imperial College London \\
\hline Kline & John & Los Alamos National Laboratory \\
\hline Knauer & James & Laboratory for Laser Energetics, University of Rochester \\
\hline Koenig & Michel & $\begin{array}{l}\text { Laboratoire pour I'Utilisation des Lasers Intenses / Centre } \\
\text { national de la recherche scientique }\end{array}$ \\
\hline Kruer & William & Lawrence Livermore National Laboratory \\
\hline
\end{tabular}




\begin{tabular}{|c|c|c|}
\hline Krushelnick & Karl & University of Michigan \\
\hline Lamb & Don & University of Chicago \\
\hline Lee & Richard & Linac Coherent Light Source \\
\hline Leeper & Ramon & Sandia National Laboratories \\
\hline Levedahl & William & National Nuclear Security Administration \\
\hline $\mathrm{Li}$ & Chikang & Massachusetts Institute of Technology \\
\hline Lindl & John & Lawrence Livermore National Laboratory \\
\hline Logan & Grant & Lawrence Berkeley National Laboratory \\
\hline Loubeyre & Paul & Commissariat á l"Energie Atomique \\
\hline Luu & Thomas & Lawrence Livermore National Laboratory \\
\hline MacLaren & Steve & Lawrence Livermore National Laboratory \\
\hline Mancini & Robert & University of Nevada Reno \\
\hline Marinak & Michael & Lawrence Livermore National Laboratory \\
\hline Maron & Yitzhak & Weizmann Institute of Science \\
\hline Mattsson & Thomas & Sandia National Laboratories \\
\hline Matzen & Keith & Sandia National Laboratories \\
\hline Mauche & Christopher & Lawrence Livermore National Laboratory \\
\hline McKenty & Patrick & Laboratory for Laser Energetics \\
\hline Mercer-Smith & James & Los Alamos National Laboratory \\
\hline Meyerhofer & David & Laboratory for Laser Energetics \\
\hline Michel & Pierre & Lawrence Livermore National Laboratory \\
\hline Mikaelian & Karnig & Lawrence Livermore National Laboratory \\
\hline Miles & Aaron & Lawrence Livermore National Laboratory \\
\hline Millot & Marius & University of California, Berkeley \\
\hline Montgomery & David & Los Alamos National Lab \\
\hline More & Richard & Lawrence Berkeley National Laboratory \\
\hline Mori & Warren & University of California, Los Angeles \\
\hline Moses & Edward & Lawrence Livermore National Laboratory \\
\hline Moses & Gregory & University of Wisconsin-Madison \\
\hline Murakami & Masakatsu & Institute of Laser Engineering \\
\hline Murphy & Thomas & Los Alamos National Laboratory \\
\hline Myatt & Jason & Laboratory for Laser Energetics, University of Rochester \\
\hline Nakhleh & Charles & Sandia National Laboratories \\
\hline Nuckolls & John & Lawrence Livermore National Laboratory \\
\hline Obenschain & Stephen & Naval Research Laboratory \\
\hline Olson & Rick & Sandia National Laboratories \\
\hline Patel & Mehul & Lawrence Livermore National Laboratory \\
\hline Perkins & John L & Lawrence Livermore National Laboratory \\
\hline Pudliner & Brian & Lawrence Livermore National Laboratory \\
\hline Quintenz & Jeffrey & National Nuclear Security Administration \\
\hline Randewich & Andrew & Atomic Weapons Establishment \\
\hline Regan & Sean & Laboratory for Laser Energetics, University of Rochester \\
\hline Robey & Harry & Lawrence Livermore National Laboratory \\
\hline
\end{tabular}




\begin{tabular}{|c|c|c|}
\hline Rosen & Paula & Atomic Weapons Establishment \\
\hline Rosen & Mordecai & Lawrence Livermore National Laboratory \\
\hline Rosner & Robert & University of Chicago \\
\hline Roth & Markus & $\begin{array}{l}\text { Technical University Darmstadt / Los Alamos National } \\
\text { Laboratory }\end{array}$ \\
\hline Rozmus & Wojciech & University of Alberta \\
\hline Sangster & Craig & Laboratory for Laser Energetics \\
\hline Schmitt & Andrew & Naval Research Laboratory \\
\hline Scott & Howard & Lawrence Livermore National Laboratory \\
\hline Sefkow & Adam & Sandia National Laboratories \\
\hline Sepke & Scott & Lawrence Livermore National Laboratory \\
\hline Sherrill & Manolo & Los Alamos National Laboratory \\
\hline Shumlak & Uri & University of Washington \\
\hline Sinars & Daniel & Sandia National Laboratories \\
\hline Springer & Paul & Lawrence Livermore National Laboratory \\
\hline Sterne & Philip & Lawrence Livermore National Laboratory \\
\hline Stevenson & Richard & Atomic Weapons Establishment \\
\hline Synakowski & Edmund & Department of Energy \\
\hline Takabe & Hideaki & Osaka University \\
\hline Tarter & Bruce & Lawrence Livermore National Laboratory \\
\hline Vesey & Roger & Sandia National Laboratories \\
\hline Vogman & Genia & University of California, Berkeley \\
\hline Vogt & Rochus (Robbie) & Caltech/ LLNS BOG STC \\
\hline Wallin & Brad & Lawrence Livermore National Laboratory \\
\hline Wan & Alan & Lawrence Livermore National Laboratory \\
\hline Wark & Justin & University of Oxford \\
\hline Wiley & Larry & Lawrence Livermore National Laboratory \\
\hline Wilson & Douglas & Los Alamos National Laboratory \\
\hline Wootton & Alan & University of Texas \\
\hline Wurtele & Jonathan & Department of Physics \\
\hline Zarnstorff & Michael & Princeton Plasma Physics Laboratory \\
\hline Zimmerman & George & Lawrence Livermore National Laboratory \\
\hline
\end{tabular}




\section{APPENDIX C: PANEL SCOPE DESCRIPTIONS}




\section{Panel 1: Laser Propagation and X-ray Production}

The "outer" NIF laser beams enter the hohlraum, propagate towards, and primarily heat, the Au wall. They are collisionally absorbed in sub critical gold plasma, and electron thermal conduction then heats denser gold, which is the primary source of xray production. The x-rays are mostly sub keV ("capsule drive") photons, plus "M-band" (2-4 keV photons) that have capsule preheat implications. The "inner" beams, on their way to the Au wall near the hohlraum midplane, must traverse a longer path of plasma, much of which consists of both low Z "hohlraum fill gas" as well as rarefied, heated, capsule ablator material.

There are many issues involved in properly modeling the large number of physical processes involved in this narrative. Due to beam overlap in the plasma, there is transfer of power from outer to inner beams. This time dependent energy transfer is being used for capsule implosion symmetry control. Accurately calculating this crossbeam transfer requires knowledge of the plasma conditions (T, n, velocity field) near the Laser Entrance Hole (LEH), as well as a good theory for the saturation mechanisms for the transfer.

As the beams propagate they are collisionally absorbed. Accurately calculating the absorption also requires knowledge of the $\mathrm{T}, \mathrm{n}$, and $\mathrm{Z}$ of the plasma. The non-LTE radiation processes, the non-local electron conduction, and the non-Maxwellian particle distributions all affect these quantities. The mixing of the high $\mathrm{Z}$ Au blow-off plasma from the wall with the low $Z$ fill gas plasma can also be a factor in determining these quantities. The LEH itself is dynamically closing, and difficult to model properly, and magnetic fields are likely to form within its sharp gradients. The LEH affects laser beam propagation inward, as well as "Dante", the x-ray drive diagnostic which gathers x-rays emitted through that LEH.

Laser Plasma Instabilities (LPI) also occur. Stimulated Brillouin (SBS) and Raman (SRS) instabilities scatter the light off of ion and electron waves respectively. The SRS electron plasma wave can produce hot electrons that can preheat the capsule directly, or possibly indirectly through inducing high-energy photons from the Au wall. Accurately calculating these LPI processes has been a multi-decade, and multi-scale enterprise. Accurately knowing the plasma conditions and the local laser intensity, are necessary (though possibly insufficient) inputs to understanding what LPI to expect. As but one example of the non-linear cross-talk amongst all of the above, consider this: Cross beam transfer can intensify just a part of an inner beam. The resulting SRS from that intense part of the inner beam can send light back, from the interior of the hohlraum, towards the LEH. That scattered light can be collisionally absorbed and heat the plasma in the LEH, thus changing the plasma conditions that can then affect the cross beam transfer.

In this panel we will raise issues as to how to better model (and better diagnose experimentally) this panoply of issues, and discuss the implications thereof for better ignition target performance. 


\section{Panel 2: X-ray transport and ablation physics}

This panel will examine the physics that drives the "1D" hydrodynamics of NIC implosions, namely the x-ray transport in an indirect-drive hohlraum and the ablation of the capsule. Of particular interest are the coupling efficiency and drive pressure history of the capsule. The data show there are physics issues that need to be understood further.

NIC capsule implosions show discrepancies between models employing the nominal xray drive and observed global hydrodynamic properties such as velocity history, bang time and shell thickness. Inferred stagnation pressures are lower than predicted, suggesting that extra entropy is injected in the capsule hot spot that inhibits compression, possibly due to the global hydrodynamics, such as an extra shock. These discrepancies affect every aspect of the implosion; symmetry, stability, and mix. Understanding these effects and developing predictive models will allow us to design more optimal implosions. Sources of these discrepancies include the possibility that the x-ray drive seen by the capsule differs in unexpected ways from that observed through the laser entrance hole (LEH), and that the ablator response to the drive through material properties (equation of state or opacity) differs from the models.

The drive observed through the entrance hole with the Dante diagnostic is expected to be different from that seen by the capsule. Designers use integrated calculations of the hohlraum and capsule to connect them, but this depends on calculations of the distributions of hot, $\mathrm{x}$-rayemitting and cooler, absorbing matter that might shadow the capsule. As mentioned in the scope for Panel 1, mix of gas fill with the ablated hohlraum wall may alter those distributions. Recent data suggest that while models may match the measured DANTE signal they over-predict LEH closure, suggesting the actual radiation temperatures through the LEH and on the capsule are lower than simulated.

The ablator material properties play a critical role in capsule performance. Much of the NIC tuning campaign has been focused on adjusting for uncertainties in these properties. Different equation of state and opacity models as well as potential non-Local Thermodynamics Equilibrium (non-LTE) properties of the polymer ablator remain of active interest. Modeling with the Cretin non-LTE code shows that carbon in the low-density blow off can have an ionization state significantly higher that LTE predictions due to the high photon flux. The ionization energy of K-shell electrons of carbon is comparable to the characteristic photon energy of the incident $\mathrm{X}$-ray spectrum as the radiation temperature rises through the third and fourth pulses. In that regime, K-shell physics plays an important part in the energy balance, so differences in ionization state or level populations can affect ablator performance. Initial nonLTE models suggest as much as half of the timing discrepancy in capsule implosion time could be due to non-LTE effects, although a robust non-LTE model remains a work in progress. Some of the models show an undesirable double-ablation front structure during part of the drive, eventually collapsing into a single front that could launch an additional shock.

This panel will explore ways to improve understanding of the radiation transport to the ablation front and the way the ablator responds to that incident radiation flux. We will consider modeling improvements and experiments that could help unravel the source of discrepancies between models and NIC implosions. 
Panel 3: Implosion hydrodynamics

A challenge that the NIF ignition experiments are facing right now is low hot -spot pressure at stagnation and low neutron yield. This can result from a combination of 1D (fuel adiabat) and 3D effects (asymmetry, mix).

The hot-spot pressure scales with ablation pressure $\mathrm{p}_{\mathrm{q}}$, implosion velocity $\mathrm{V}_{\mathrm{imp}}$, and fuel adiabat $\alpha$ as $p_{m} \sim p_{a}^{\frac{1}{3}} V_{i m p}^{3} \alpha^{-1}$.

The fuel adiabat is set by properly timing four shocks at the beginning of an implosion. Matching the shock timing data with direct numerical simulations, however, requires using either time-dependent reduction factors on the laser energy or a "switch" between LTE and non-LTE models in gold which is set to $75 \mathrm{eV}$ to match velocities of the shocks launched by the second and third pickets. Only LTE tables are used to simulate velocity of the first shock. This threshold, however, might be dependent on resolution of the region where the laser interacts with the hohlraum wall. A self-consistent model explaining drive pressure evolution consistent with shock velocity data is desirable since it's not clear that the adjustment factors predict correct hydro profiles and drive pressure evolution during the main drive.

The strength of the $4^{\text {th }}$ shock is much weaker than in simulations, and the final implosion velocity is $5-10 \%$ lower than predicted (corresponding to $\sim 10-20 \%$ effective reduction in the drive the capsule experiences). The observed strength of the fourth shock can be matched in these standard simulations only if the incident laser power is reduced substantially during the rising edge of the $4^{\text {th }}$ pulse so that the predicted X-ray drive on the capsule rises more slowly than predicted. Currently, no model can explain entirely these observations.

Additional degradation of the shell adiabat could come from extra steepening in the adjustment compression wave, which is generated at the beginning of shell acceleration after all four shocks break out of the shell. Such a compression wave does not turn into a strong shock in an optimized design. In a scenario when the fourth shock is weak, the adjustment wave might be traveling through more relaxed density and pressure gradients and turn into a stronger $5^{\text {th }}$ shock.

Loss in drive pressure leads to shell decompression and a reduction in the stagnation pressure, as suggested by the scaling shown in (1). The experimentally measured timing of $\mathrm{x}$ ray emission at peak compression is always late compared to predictions. This suggests that the drive is reduced. At present time, there is no clear explanation for such a reduction. Possible scenarios include LEH dynamics, change in plasma opacity due to mix of shell blowoff with wall blowoff, and sidescaterring due to LPI.

Low neutron yield and stagnation pressure can also be explained by an increase in the volume of the vapor region due to multi-dimensional effects (low-1 mode hot spot distortion and mix). Current calculations of the hot-spot distortion growth do not include effects of magnetic fields which become important for highly distorted implosions. Including these fields is important step in understanding the hot-spot formation.

This group will focus on what the dominant physics issues appear to be, how we might unravel the importance of each through analysis of existing data, new experiments or measurements, and how to better model them. 


\section{Panel 4: Stagnation and Burn}

To achieve hot-spot ignition and energy gain using the indirect-drive approach at the NIF, it is essential to set the correct conditions for the acceleration, coasting and deceleration phase of an implosion. Stringent requirements on the design of the cryogenically layered DT capsule and drive are therefore critical to obtain correct stagnation properties for optimal burn. This requires excellent understanding of the underlying physics that dictates and connects the:

- $\quad$ Strength and timing of the four sequential shocks used to compress the DT ice layer and set the fuel compressibility without significantly raising the fuel adiabat.

- Acceleration of the DT layer to highest possible velocity for maximum implosion kinetic energy while maintaining mix under control.

- Minimization of the coasting phase, which can decompress the DT fuel, raise the stagnation adiabat and the minimum kinetic energy required for ignition.

- Hot-spot formation during the deceleration phase and stagnation. Here, it is important to effectively convert the kinetic to thermal energy while at the same time minimizing the effect of mix.

- $\quad$ Alpha particle transport through the hot sport and high-density fuel for maximum bootstrap heating and effective burn propagation, which dictates the ignition threshold and energy gain respectively.

There are many issues involved in properly understanding the physical processes associated with each phase of the implosion, all of which play a critical role for setting the correct stagnation properties for optimal burn. Accurate modeling of the physics connecting the drive pressure, stagnation adiabat and energy required for ignition is therefore required. In current NIF experiments, there are still issues in forming the hot spot. The yield and pressures are lower than post-shot simulations and the hot-spot entropy is too high (large volume, small pressure and small mass), which might be due to shock mistiming (the fourth rise is causing hot release of inner ice layer), a fifth shock, inefficient conversion of kinetic to thermal energy, early termination of drive that increases the coasting phase, hydro jets caused by the fill tube and/or ice inhomogeneity.

In this panel, we will discuss how to better model (and better diagnose experimentally) this panoply of issues, and discuss the implications thereof for better implosion performance. 


\section{Panel 5: High Energy Density Matter Crosscut}

Ignition implosions access an unprecedented range of high energy density (HED) states. In order to simulate and interpret these implosions a sufficiently accurate and complete set of materials data are needed. This panel will interrogate current models used "in-line" in ICF calculations, and discuss a plan to evolve a next-generation of benchmarked physics models expressly focused on the interpretation of experiments and optimization of future fusion designs. Physics areas include equation of state (EOS), Opacity (local thermodynamic equilibrium, LTE, and non-thermodynamic equilibrium, non-LTE), nuclear cross sections, nuclear decay spectra, kinetics, electric and magnetic field generation, and several transport quantities such as thermal conduction, electron-ion equilibration, ion stopping power, and viscosity.

Starting from an ignition hohlraum, 1.3 MJ of laser energy is converted to $1 \mathrm{MJ}$ of $\mathrm{x}$-ray energy through the collisional absorption of laser energy. Losses include scattered light and the acceleration of non-thermal high-energy electrons produced by laser plasma instabilities (PLI). These "hot-electrons" can pre-heat the capsule, making ignition more difficult. The detailed spectral dependence of the absorption and emission of x-ray energy requires detailed opacity (LTE and non-LTE), equation of state, and electron transport for a wide range of hohlraum states.

The transport of $\mathrm{x}$-ray energy from the hohlraum wall to the capsule results in $\sim 120 \mathrm{~kJ}$ of energy at the ablation front. Modeling the absorption and propagation of x-rays through the mix of hohlraum blow off and ablated plasma from the capsule requires LTE and non-LTE opacities and EOS's for both pure and mixed regions of materials. Modeling capsule ablation relies on accurate x-ray absorption, re-emission, ionization, and sound speed for matter near the ablation front. These quantities set the ablation velocity which lead to an imploding shell (fuel) kinetic energy of $\sim 20 \mathrm{~kJ}(12 \mathrm{~kJ})$.

During the early stages of the implosion, the x-ray energy increases with time, driving a series of shock waves through the ablator and into the DT fuel, so as to compress the cold DT to a dense-Fermi-degenerate layer on the interior of the ablator shell and then accelerate the ablator and fuel to peak velocities of near $370 \mathrm{~km} / \mathrm{s}$ with the ablator and fuel reaching $10-20 \mathrm{~g} / \mathrm{cc}$ and $\sim 10 \mathrm{eV}$. During this phase, the stability of the fuel-ablator interface is set by the density profile of the ablator and fuel, which are set by the $\mathrm{x}$-ray absorption, thermal transport, and equation of state. At stagnation, the hot spot $(100 \mathrm{~g} / \mathrm{cc}, 5 \mathrm{keV})$ is tuned to a size and density to be able to stop alpha particles $(\sim 300 \mathrm{mg} / \mathrm{cc})$. The dense fuel $(1 \mathrm{~kg} / \mathrm{cc}, 50 \mathrm{eV})$ (and remaining ablator) is tuned to allow enough inertial confinement time for hotspot initiation and setting up a propagating burn wave. Needless to say, there is not much data to constrain physics models at these conditions. The successful hotspot initiation is a race depending on many transport properties. Thermal transport from the hotspot into the cold fuel, electron-ion equilibration, ion stopping power, nuclear reaction rates, and x-ray emission/absorption.

Of course many other physical processes may be important in correctly optimizing ignition implosions such as ion kinetics, large electric or magnetic fields, viscosity etc. While significant effort was made to ensure that the materials models used in designing ICF capsules were good enough, more remain untested over the full range of extreme conditions accessed by the ICF implosion. 


\section{Panel 6: Integrated Modeling}

The simulation codes currently used to design and analyze ignition experiments include HYDRA, LASNEX, Draco, Lilac, RAGE, FAST3D, Allegra, and CHIC. Physical processes modeled in the codes include radiation transport, electron and ion thermal transport, thermonuclear burn and transport of burn products. These codes include models for transport of laser light and the various processes which affect it. They also include magnetohydrodynamics and the effects of magnetic fields on transport processes. Ingredients in the integrated models include atomic physics, in particular models for opacities and equations of state, both LTE and non-LTE. Approaches that include kinetic, and non-fluid effects are being investigated.

We will discuss what developments would have the greatest impact on improving the state of the art in modeling ignition experiments, in part guided by existing data. Among the questions we will consider are the following. How can the computational power of future platforms be exploited? Which approximations made in the models significantly affect the accuracy of the simulations and how can these models be improved? Are there processes not treated in our models which significantly affect simulation results? Are there new approaches for modeling physical processes that would substantially benefit the simulation codes? Are there better ways to verify and validate the simulation codes? 


\section{APPENDIX D: ACRONYMS}

CBET

CEA

ConA

DAC

DCA

DFT

DIM

DNS

DR

DT

EOS

FRIB

GDP

GFMC

GXD

HFM

HED

HPC

ICF

IPD

LCLS

LEH

LLNL

LPI

LSP

LTE

MRS

NCSM

NIC

NIF

non-LTE

NNSA

nTOF

PIC cross beam energy transfer

Commissariat a l'Energie Atomique

convergent ablator

diamond anvil cell

Detailed Configuration Accounting

density functional theory

Diagnostic Instrument Manipulator

Direct Numerical Simulation

dielectronic recombination

deuterium/tritium

equation of state

for rare isotope beams

glow discharge polymer

Green's function Monte Carlo

Gated X-ray Detector

high flux model

high energy density

high performance computing

inertial confinement fusion

ionization potential depression

Linac Coherent Light Source

laser entrance hole

Lawrence Livermore National Laboratory

laser-plasma interaction

large scale plasma

local thermodynamic equilibrium

Magnetic Recoil Spectrometer

no-core shell model

National Ignition Campaign

National Ignition Facility

non-local thermodynamic equilibrium

National Nuclear Security Administration

Neutron Time of Flight

particle in cell 


$\begin{array}{ll}\text { PIMC } & \text { path integral Monte Carlo } \\ \text { PRD } & \text { Priority Research Direction } \\ \text { QCD } & \text { quantum chromo-dynamics } \\ \text { RM } & \text { Richtmyer-Meshkov } \\ \text { RT } & \text { Rayleigh-Taylor } \\ \text { SBS } & \text { stimulated Brillouin scattering } \\ \text { SRS } & \text { stimulated Raman scattering } \\ \text { THD } & \text { tritium/hydrogen/deuterium } \\ \text { TS } & \text { Thompson scattering } \\ \text { VISAR } & \text { Velocity Interferometer System for Any Reflector } \\ \text { YOC } & \text { yield over clean }\end{array}$




\section{APPENDIX E: BIBLIOGRAPHY}

The following list includes representative peer-reviewed, journal publications from 2010 through May 2012 that are germane to the various Panel scopes. Additional article references are available from the Workshop website at:

○ https://lasers.llnl.gov/workshops/science_of_ignition/panel_material/

The following IOP papers are overview summaries on the status of the National Ignition Campaign at the time of publication.

Ignition and Inertial Confinement Fusion at The National Ignition Facility Edward I. Moses, Journal of Physics: Conference Series 244012006 (2010)

Progress towards ignition on the National Ignition Facility J.D. Lindl et al., Nuclear Fusion 51094024 (2011)

AIP Physics of Plasmas journal: the following representative papers, published since 2010, are in order of Vol. and Issue; the listing does not include papers 'submitted to' or 'in press.'

Capsule performance optimization in the National Ignition Campaign O. L. Landen, T. R. Boehly, D. K. Bradley, D. G. Braun, D. A. Callahan, P. M. Celliers, G. W. Collins, et al. Phys. Plasmas 17, 056301 (2010)

Experimental basis for laser-plasma interactions in ignition hohlraums at the National Ignition Facility D. H. Froula, L. Divol, R. A. London, R. L. Berger, T. Döppner, N. B. Meezan, J. Ralph, J. S. Ross, L. J. Suter, and S. H. Glenzer Phys. Plasmas 17, 056302 (2010)

National Ignition Campaign Hohlraum energetics N. B. Meezan, L. J. Atherton, D. A. Callahan, E. L. Dewald, S. Dixit, E. G. Dzenitis, M. J. Edwards, C. A. Haynam, D. E. Hinkel, et al., Phys. Plasmas 17, 056304 (2010)

Symmetry tuning via controlled crossed-beam energy transfer on the National Ignition Facility P. Michel, S. H. Glenzer, L. Divol, D. K. Bradley, D. Callahan, S. Dixit, S. Glenn, D. Hinkel, R. K. Kirkwood, J. L. Kline, et al., Phys. Plasmas 17, 056305 (2010)

Probing high areal-density cryogenic deuterium-tritium implosions using downscattered neutron spectra measured by the magnetic recoil spectrometer J. A. Frenje, D. T. Casey, C. K. Li, F. H. Séguin, R. D. Petrasso, V. Yu. Glebov, P. B. Radha, T. C. Sangster, D. D. Meyerhofer, et al., Phys. Plasmas 17, 056311 (2010)

Shock-Tuned Cryogenic-Deuterium-Tritium Implosion Performance on Omega Sangster, T. C., V. N. Goncharov, et al., Phys. Plasmas 17, 056312 (2010)

Convergent ablator performance measurements D. G. Hicks, B. K. Spears, D. G. Braun, R. E. Olson, C. M. Sorce, P. M. Celliers, G. W. Collins, and O. L. Landen, Phys. Plasmas 17, 102703 (2010)

Point design targets, specifications, and requirements for the 2010 ignition campaign on the National Ignition Facility S. W. Haan, J. D. Lindl, D. A. Callahan, D. S. Clark, J. D. Salmonson, B. A. Hammel, L. J. Atherton, R. C. Cook, M. J. Edwards, et al..Phys. Plasmas 18, 051001 (2011) 
Capsule implosion optimization during the indirect-drive National Ignition Campaign O. L. Landen, J. Edwards, S. W. Haan, H. F. Robey, J. Milovich, B. K. Spears, S. V. Weber, D. S. Clark, J. D. Lindl, et al., Phys. Plasmas 18, 051002 (2011)

The experimental plan for cryogenic layered target implosions on the National Ignition Facility - The inertial confinement approach to fusion M. J. Edwards, J. D. Lindl, B. K. Spears, S. V. Weber, L. J. Atherton, D. L. Bleuel, D. K. Bradley, D. A. Callahan, C. J. Cerjan, et al., Phys. Plasmas 18, 051003 (2011)

Analysis of the National Ignition Facility ignition hohlraum energetics experiments R. P. J. Town, M. D. Rosen, P. A. Michel, L. Divol, J. D. Moody, G. A. Kyrala, M. B. Schneider, J. L. Kline, C. A. Thomas et al., Phys. Plasmas 18, 056302 (2011)

Symmetry tuning for ignition capsules via the symcap technique G. A. Kyrala, J. L. Kline, S. Dixit, S. Glenzer, D. Kalantar, D. Bradley, N. Izumi, N. Meezan, O. Landen, D. Callahan, S. V. Weber, et al., Phys. Plasmas 18, 056307 (2011)

Stimulated Raman scatter analyses of experiments conducted at the National Ignition Facility D. E. Hinkel, M. D. Rosen, E. A. Williams, A. B. Langdon, C. H. Still, D. A. Callahan, J. D. Moody, P. A. Michel et al., Phys. of Plasmas 18, 056312 (2011)

The potential role of electric fields and plasma barodiffusion on the inertial confinement fusion database Peter Amendt, S. C. Wilks, C. Bellei, C. K. Li, and R. D. Petrasso, Phys. Plasmas 18, 056308 (2011)

Diagnosing and controlling mix in National Ignition Facility implosion experiments B. A. Hammel, H. A. Scott, S. P. Regan, C. Cerjan, D. S. Clark, M. J. Edwards, R. Epstein, S. H. Glenzer, S. W. Haan, N. Izumi, et al., Phys. Plasmas 18, 056310 (2011)

Kinetic simulations of stimulated Raman backscattering and related processes for the shock-ignition approach to inertial confinement fusion C. Riconda, S. Weber, V. T. Tikhonchuk, and A. HéronPhys. Plasmas 18, $092701(2011)$

Multiple spherically converging shock waves in liquid deuterium T. R. Boehly, V. N. Goncharov, W. Seka, S. X. Hu, J. A. Marozas, D. D. Meyerhofer, P. M. Celliers, D. G. Hicks, M. A. Barrios, et al., Phys. Plasmas 18, 092706 (2011)

Shock timing experiments on the National Ignition Facility: Initial results and comparison with simulation H. F. Robey, T. R. Boehly, P. M. Celliers, J. H. Eggert, D. Hicks, R. F. Smith, R. Collins, M. W. Bowers, K. G. Krauter, P. S. Datte, et al., Phys. Plasmas 19, 042706 (2012)

The velocity campaign for ignition on NIF D. A. Callahan, N. B. Meezan, S. H. Glenzer, A. J. MacKinnon, L. R. Benedetti, D. K. Bradley, J. R. Celeste, P. M. Celliers, et al., Phys. Plasmas 19, 056305 (2012)

Hotspot mix in ignition-scale implosions on the NIF S. P. Regan, R. Epstein, B. A. Hammel, L. J. Suter, J. Ralph, H. Scott, M. A. Barrios, D. K. Bradley, D. A. Callahan, C. Cerjan, et al.Phys. Plasmas 19, 056307 (2012)

Trapping induced nonlinear behavior of backward stimulated Raman scattering in multi-speckled laser beams L. Yin, B. J. Albright, H. A, Rose, K. J. Bowers, B. Bergen, R. K. Kirkwood, D. E. Hinkel, A. B. 
Langdon, P. Michel, et al., Phys. Plasmas 19, 056304 (2012)

Investigating inertial confinement fusion target fuel conditions through X-ray spectroscopy Stephanie B. Hansen, Phys. Plasmas 19, 056312 (2012)

APS Physical Review Letters journal: the following representative papers, published since 2010, are in order of Vol. and Issue; the listing does not include papers 'submitted to' or 'in press.'

,Generalized Measurable Ignition Criterion for Inertial Confinement Fusion Chang Py et al., 2010 Phys. Rev. Lett. 104135002

Demonstration of the Highest Deuterium-Tritium Areal Density Using Multiple-Picket Cryogenic Designs on OMEGA Goncharov, V. N., T. C. Sangster et al., 2010 Phys. Rev. Lett. 104, 165001

Observation of High Soft X-Ray Drive in Large-Scale Hohlraums at the National Ignition Facility Kline J L et al. 2011 Phys. Rev. Lett. 106085003

Demonstration of Ignition Radiation Temperatures in Indirect-Drive Inertial Confinement Fusion Hohlraums Glenzer S H et al., 2011 Phys. Rev. Lett. 106085004

Other journal articles: the following representative papers, published since 2010, are in order of Vol. and Issue; the listing does not include papers 'submitted to' or 'in press.'

The role of a DCA atomic physics package in explaining the energy balance in ignition scale hohlraums M.D. Rosen, H. A. Scott, D. E. Hinkel, E. A. Williams, D. A. Callahan, R. P. J. Town, L. Divol, P. A. Michel, W. L. Kruer et al., High Energy Density Physics 7, (3), 180 (2011)

Multi-step redirection by cross-beam power transfer of ultra-high-power lasers in a plasma J. D. Moody, P. Michel, L. Divol, R. L. Berger, E. Bond, D. K. Bradley, D. A. Callahan, E. L. Dewald, S. Dixit, S. Glenn et al., Nature Physics 8, 344 (2012) 
